\title{
Mathematical Model of Three Age-Structured Transmission Dynamics of Chikungunya Virus
}

\author{
Folashade B. Agusto, ${ }^{1}$ Shamise Easley, ${ }^{2}$ Kenneth Freeman, ${ }^{2}$ and Madison Thomas ${ }^{2}$ \\ ${ }^{1}$ Department of Ecology and Evolutionary Biology, University of Kansas, Lawrence, KS 66045, USA \\ ${ }^{2}$ Department of Mathematics and Statistics, Austin Peay State University, Clarksville, TN 37044, USA \\ Correspondence should be addressed to Folashade B. Agusto; fbagusto@gmail.com
}

Received 24 November 2015; Revised 31 December 2015; Accepted 3 January 2016

Academic Editor: Francesco Pappalardo

Copyright (c) 2016 Folashade B. Agusto et al. This is an open access article distributed under the Creative Commons Attribution License, which permits unrestricted use, distribution, and reproduction in any medium, provided the original work is properly cited.

\begin{abstract}
We developed a new age-structured deterministic model for the transmission dynamics of chikungunya virus. The model is analyzed to gain insights into the qualitative features of its associated equilibria. Some of the theoretical and epidemiological findings indicate that the stable disease-free equilibrium is globally asymptotically stable when the associated reproduction number is less than unity. Furthermore, the model undergoes, in the presence of disease induced mortality, the phenomenon of backward bifurcation, where the stable disease-free equilibrium of the model coexists with a stable endemic equilibrium when the associated reproduction number is less than unity. Further analysis of the model indicates that the qualitative dynamics of the model are not altered by the inclusion of age structure. This is further emphasized by the sensitivity analysis results, which shows that the dominant parameters of the model are not altered by the inclusion of age structure. However, the numerical simulations show the flaw of the exclusion of age in the transmission dynamics of chikungunya with regard to control implementations. The exclusion of age structure fails to show the age distribution needed for an effective age based control strategy, leading to a one size fits all blanket control for the entire population.
\end{abstract}

\section{Introduction}

Chikungunya is a viral disease that is transmitted to humans from an infected mosquito of the Aedes genus (particularly the Aedes aegypti and Aedes albopictus mosquitoes $[1,2]$ ). It is an RNA virus that belongs to Alphavirus genus of the family Togaviridae [3]. It was first described about 1952 during an outbreak in southern Tanzania [3]. Chikungunya in the Kimakonde language (the language from where the name was derived) means to become contorted or "bend over" [3]. There have been numerous cases of reemergence of chikungunya in Africa, Asia, Europe, and more recently the Caribbean [4]. The virus was isolated in 1960s in Bangkok and in 1964, the virus resurfaced in parts of India including Vellore, Calcutta, and Maharastha [5]. Other outbreaks include Sri Lanka in 1969, Vietnam in 1975, Myanmar in 1975, and Indonesia in 1982 [5]. A large outbreak occurred in the Democratic Republic of the Congo in 1999-2000 [3]. In the years 2005-2007, an outbreak occurred in the islands of the Indian Ocean. Gabon was hit with an outbreak in 2007 [3]. Since 2005, India, Indonesia, Thailand, Maldives, and Myanmar have encountered over 1.9 million cases [3]. The disease spread to Europe by 2007 with 197 cases being recorded [3]. More recently, in December 2013, the French part of the Caribbean island of St. Martin reported two laboratory-confirmed autochthonous (native) cases [3, 4]. Since then, local transmission have been confirmed in the Dutch part of Saint Martin (St Maarten), Anguilla, British Virgin Islands, Dominica, French Guiana, Guadeloupe, Martinique, and St Barthelemy [3]. As of October 2014, over 776,000 suspected cases of chikungunya have been recorded in the Caribbean islands, Latin American countries, and some south American countries [3]. About 152 deaths have also been attributed to the disease during the same period. Mexico 
and USA have also recorded imported cases. On October 21, 2014, France confirmed four cases of chikungunya locally acquired infection in Montpellier, France [3].

In 2005-2006, a major chikungunya outbreak involving numerous islands in the Indian Ocean (notably La Reunion Island) occurred; one-third of the population were infected [6]. According to Schuffenecker et al. [7] and Vazeille et al. [8], in two concurrent studies, the chikungunya virus strains in the Reunion Island outbreak mutated to facilitate the disease transmission by Aedes albopictus (Tiger mosquito) $[6,9]$. The mutation was a point mutation in one of the viral envelope genes (E1 glycoprotein gene (E1-226V)) [10,11]. Dubrulle et al. [6] found that this mutation allowed the virus to be present in the mosquito saliva only two days after the infection, instead of approximately seven days in the Aedes aegypti mosquitoes. This shows that Aedes albopictus is a slightly more efficient host than Aedes aegypti in transmitting the variant E1-226V of chikungunya virus. Hence, this result indicates that other areas where the tiger mosquitoes are present could be at greater risk of outbreak with an enhanced transmission of chikungunya virus by Aedes albopictus.

Following an effect bite (i.e., a bite leading to an infection) from infected mosquitoes $[1,2]$, the incubation period is usually within 3-7 days; symptoms include fever, headache, nausea, fatigue, rash, and severe joint pain (including lower back, ankle, knees, wrists, or phalanges) $[1,2]$. There is no antiviral medicine to treat the disease $[1,2]$; all the treatments are directed at relieving the disease symptoms [3]. There is no preventative vaccine for chikungunya [3]; however, findings of an experimental vaccine in an early-stage clinical trial are promising; it prompted an immune response in all 25 volunteers [12].

Chikungunya rarely results in death and infected individuals are expected to make full recovery with life-long immunity [2]. However there are some cases where individuals experience joint pains for several months or years after the initial infection [3]. There have also been reports of eye, neurological, and heart complications and gastrointestinal complaints [3]. The disease symptoms, generally, are mild and the infection may go unrecognized; however, several studies [1316 ] have shown that children (especially neonates), the elderly ( $\geq 65$ years), and people with medical conditions (such as high blood pressure, diabetes, or heart disease) have more severe clinical manifestation of chikungunya than in older children and adults ( $<65$ years). The severity of the symptoms can be described by a U-shaped curve, with a maximum occurring in young infants and the elderly and a minimum in older children [16]. Furthermore, the rate of asymptomatic infection among children varies according to different outbreak reports (range 35-40\%) [16]; overall, approximately 3-28\% of infected individuals will remain asymptomatic $[17,18]$. In the study of the 2006-2007 chikungunya epidemic in Kerala, South India, Vijayakumar et al. [19] showed an age distribution of people affected with chikungunya. Their study indicates that the adult group (ages 15-59) were the most affected age group; they consist of about $73.4 \%$ of the entire study population; this is followed by the elderly group (ages $>60$ ); this group make up about $15.6 \%$ of the study population. Finally, $11 \%$ of the cases occurred in persons ages $<15$ years. Similar age distribution was reported in other epidemics in India [20], Thailand [21], and Reunion Islands [5, 22] and across Europe [23] (including Austria, Czech Republic, Estonia, Finland, France, Germany, Greece, Hungary, Ireland, Italy, Latvia, Lithuania, Luxembourg, Malta, Poland, Romania, Slovakia, Slovenia, Spain, Sweden, and United Kingdom).

A number of studies have been carried out to study the chikungunya virus, considering different factors that effect the outbreak of the disease (see [21, 24-30]). Ruiz-Moreno et al. [24] analyzed the potential risk of chikungunya introduction into the US; their study combines a climate-based mosquito population dynamics stochastic model with an epidemiological model to identify temporal windows that have epidemic risk. Dumont et al. [25] propose a model, including human and mosquito compartments, that is associated with the time course of the first epidemic of chikungunya in Reunion Island. Using entomological results, they investigated the links between the episode of 2005 and the outbreak of 2006. Manore et al. [26] investigated, via an adapted mathematical model, the differences in transient and endemic behavior of chikungunya and dengue, risk of emergence for different virus-vector assemblages, and the role that virus evolution plays in disease dynamics and risk. Poletti et al. [29] developed a chikungunya transmission model for the spread of the epidemic in both humans and mosquitoes; the model involves a temporal dynamics of vector (Aedes albopictus), depending on climatic factors. In the study, they provided estimates of the transmission potential of the virus and assessed the efficacy of the measures undertaken by public health authorities to control the epidemic spread in Italy. Yakob and Clements [30] developed a simple, deterministic mathematical model for the transmission of the virus between humans and mosquitoes. They fitted the model to the large Reunion epidemic data and estimated the type reproduction number for chikungunya; their model provided a close approximation of both the peak incidence of the outbreak and the final epidemic size. Pongsumpun and Sangsawang [21] developed and studied theoretically an agestructured model for chikungunya involving juvenile and adult human populations, giving conditions for the diseasefree and endemic states, respectively. They also suggested alternative way for controlling the disease.

The aim of this study is to develop a new deterministic transmission model to gain qualitative insight into the effects of age on chikungunya transmission dynamics and to determine the importance or otherwise of the inclusion of age in the transmission dynamics. A notable feature of the model is the incorporation of three different human age classes involving juvenile, adult, and senior human populations; the model also involves two infectious human classes, notably the asymptomatic and symptomatic classes. The paper is organized as follows; the model is formulated in Section 2, the analysis of the mathematical properties of the model is stated in Section 3. The effect of the age structure on the disease transmission is explored in Section 4 . The sensitivity analysis of the model is investigated in Section 4.1. Following the result obtained from the sensitivity analysis, various control strategies are implemented in Section 5. The key theoretical 
and epidemiological results from this study are discussed and summarized in Section 6.

\section{Model Formulation}

The model is formulated as follows with human and mosquito subgroups. The human population is divided into juvenile, adult, and senior subpopulations. The human subgroup is further divided into susceptible $\left(S_{i}\right)$, exposed $\left(E_{i}\right)$, symptomatic $\left(I_{S i}\right)$, asymptomatic $\left(I_{A i}\right)$, and recovered $\left(R_{i}\right)$, where $i=J, A, S$ for the juvenile, adult, and senior subpopulations. Thus, the total human population $N_{H}(t)=S_{J}(t)+E_{J}(t)+I_{A J}(t)+$ $I_{S J}(t)+R_{J}(t)+S_{A}(t)+E_{A}(t)+I_{A A}(t)+I_{S A}(t)+R_{A}(t)+$ $S_{S}(t)+E_{S}(t)+I_{A S}(t)+I_{S S}(t)+R_{S}(t)$. The mosquito population is divided into three classes consisting of susceptible mosquitoes $\left(S_{M}\right)$, exposed mosquitoes $\left(E_{M}\right)$, and infected mosquitoes $\left(I_{M}\right)$. Hence, the total mosquito population $N_{M}(t)=S_{M}(t)+E_{M}(t)+R_{M}(t)$.

Individuals move from one class to the other as their status evolves with respect to the disease. The population of susceptible juvenile $\left(S_{J}\right)$ is generated at the rate $\pi_{J}$ via birth or immigration. It is assumed that there is no vertical transmission or immigration of infectious humans; thus there is no inflow into the infectious classes. The population is reduced by the juvenile maturation at the rate $\alpha$ and by natural death at the rate $\mu_{J}$. The infection rate of susceptible juveniles $\lambda_{J}$ is given as

$$
\lambda_{J}=\frac{\beta_{J} b_{M} I_{M}}{N_{H}} .
$$

The parameter $\beta_{J}$ in (1) is the probability that a bite from an infectious mosquito leads to infection of the susceptible juvenile and the parameter $b_{M}$ is the mosquito biting rate. The derivation of (1) is given in Appendix A.

Similarly, it can be shown that the rate at which mosquitoes acquire infection from infectious (asymptomatic and symptomatic) human hosts is given by

$$
\lambda_{M}=\frac{\beta_{M} b_{M}\left(I_{A J}+I_{S J}+I_{A A}+I_{S A}+I_{A S}+I_{S S}\right)}{N_{H}} .
$$

The parameter $\beta_{M}$ is the probability that a bite from a susceptible mosquito to a human leads to infection of the mosquito.

Susceptible juveniles are infected by the chikungunya virus at a rate $\lambda_{J}$ and move into the exposed class. Thus, the susceptible population is given as

$$
\frac{d S_{J}}{d t}=\pi_{J}-\frac{\beta_{J} b_{M} S_{J} I_{M}}{N_{H}}-\alpha S_{J}-\mu_{J} S_{J} .
$$

The exposed juvenile population is generated following infection of the susceptible juveniles by infected mosquitoes. A fraction $\left(1-\varepsilon_{J}\right)$ of exposed juveniles enter the asymptomatic class $I_{A J}(t)$ at the rate $\left(1-\varepsilon_{J}\right) \sigma_{J}$ and the remaining fraction $\left(\varepsilon_{J}\right)$ goes into the symptomatic class $\left(I_{A S}\right)$ at the rate $\varepsilon_{J} \sigma_{J}$. The population of the exposed juvenile is reduced by the juvenile maturation at the rate $\alpha$. The exposed juvenile population is further reduced by natural death at the rate $\mu_{J}$. Thus, the exposed population is given as

$$
\frac{d E_{J}}{d t}=\frac{\beta_{J} b_{M} S_{J} I_{M}}{N_{H}}-\alpha_{J} E_{J}-\left(\sigma_{J}+\mu_{J}\right) E_{J} .
$$

Members of the juvenile asymptomatic class $I_{A J}(t)$ are generated from the fraction that moved from the juvenile exposed class. This class is reduced by maturation to the adult asymptomatic class $\left(I_{A A}\right)$ at the rate $\alpha$, by recovery (either naturally or via the use of treatment) at a rate $\gamma_{A J}$ to the recovered class. Similarly, members of the juvenile symptomatic class $I_{S J}(t)$ are populated from the fraction that moved from the juvenile exposed class. The class is reduced due to maturation to the adult symptomatic class $\left(I_{S A}\right)$ at the rate $\alpha$ and by progression to the recovered class recovery at a rate $\gamma_{S J}$. These populations are further reduced by natural death at the rate $\mu_{J}$; chikungunya rarely results in death $[1,2]$; as such we have ignored the disease induced death rate. Thus, the equations for these classes are given as follows:

$$
\begin{aligned}
& \frac{d I_{A J}}{d t}=\varepsilon_{J} \sigma_{J} E_{J}-\alpha I_{A J}-\left(\gamma_{A J}+\mu_{J}\right) I_{A J}, \\
& \frac{d I_{S J}}{d t}=\left(1-\varepsilon_{J}\right) \sigma_{J} E_{J}-\alpha I_{S J}-\left(\gamma_{S J}+\mu_{J}\right) I_{S J} .
\end{aligned}
$$

The juvenile recovered class $R_{J}$ is populated from the juvenile asymptomatic and symptomatic classes; the class is reduced by maturation to the adult class at a rate $\alpha$ and by natural death at a rate $\mu_{J}$. The equation for this class is given as follows:

$$
\frac{d R_{J}}{d t}=\gamma_{A J} I_{A J}+\gamma_{S J} I_{S J}-\alpha R_{J}-\mu_{J} R_{J} .
$$

The corresponding equations (susceptible, exposed, asymptomatic, symptomatic, and recovered) for the adult and senior classes are similarly obtained; additionally there is a maturation rate $\xi$ from the adult population into the senior class. We assume that the recovery rates from adults asymptomatic and symptomatic classes are greater than those from juvenile classes which in turn are greater than those from the senior classes (i.e., $\gamma_{A A}, \gamma_{S A}>\gamma_{A J}, \gamma_{S J}>\gamma_{A S}, \gamma_{S S}$ ) [36]. Furthermore, we assume that seniors progress more quickly to the asymptomatic and symptomatic classes than juveniles and adults (i.e., $\sigma_{S}>\sigma_{J}, \sigma_{A}$ ) [36].

The population of the susceptible mosquitoes $\left(S_{M}\right)$ is generated by the recruitment rate $\pi_{M}$ and reduced following effective contact with an infected human. All mosquitoes classes are reduced by natural death at a rate $\mu_{M}$. The equation for this class is given as follows:

$$
\begin{aligned}
\frac{d S_{M}}{d t} & \\
= & \pi_{M} \\
& -\beta_{M} b_{M}\left[\frac{I_{A J}+I_{S J}+I_{A A}+I_{S A}+I_{A S}+I_{S S}}{N_{H}}\right] S_{M} \\
& -\mu_{M} S_{M} .
\end{aligned}
$$


Mosquitoes in the exposed class $E_{M}$ are generated following the infection of the susceptible mosquitoes. They progress to the infected class at a rate $\sigma_{M}$. The equation for the exposed mosquitoes dynamics is given as follows:

$$
\begin{aligned}
\frac{d E_{M}}{d t}= & \beta_{M} b_{M}\left[\frac{I_{A J}+I_{S J}+I_{A A}+I_{S A}+I_{A S}+I_{S S}}{N_{H}}\right] S_{M} \\
& -\mu_{M} S_{M}-\sigma_{M} E_{M} .
\end{aligned}
$$

The infected mosquitoes class are populated from the exposed mosquitoes. The equation for this class is given as follows:

$$
\frac{d I_{M}}{d t}=\sigma_{M} E_{M}-\mu_{M} I_{M}
$$

Combining the aforementioned derivations and assumptions the model for the transmission dynamics of chikungunya virus in a population is given by the following deterministic system of nonlinear differential equations:

$$
\begin{aligned}
& \frac{d S_{J}}{d t}=\pi_{J}-\frac{\beta_{J} b_{M} S_{J} I_{M}}{N_{H}}-\alpha S_{J}-\mu_{J} S_{J} \\
& \frac{d E_{J}}{d t}=\frac{\beta_{J} b_{M} S_{J} I_{M}}{N_{H}}-\alpha E_{J}-\left(\sigma_{J}+\mu_{J}\right) E_{J}, \\
& \frac{d I_{A J}}{d t}=\varepsilon_{J} \sigma_{J} E_{J}-\alpha I_{A J}-\left(\gamma_{A J}+\mu_{J}\right) I_{A J}, \\
& \frac{d I_{S J}}{d t}=\left(1-\varepsilon_{J}\right) \sigma_{J} E_{J}-\alpha I_{S J}-\left(\gamma_{S J}+\mu_{J}\right) I_{S J} \\
& \frac{d R_{J}}{d t}=\gamma_{A J} I_{A J}+\gamma_{S J} I_{S J}-\alpha R_{J}-\mu_{J} R_{J} \\
& \frac{d S_{A}}{d t}=\alpha S_{J}-\frac{\beta_{A} b_{M} S_{A} I_{M}}{N_{H}}-\xi S_{A}-\mu_{A} S_{A}, \\
& \frac{d E_{A}}{d t}=\alpha E_{J}+\frac{\beta_{A} b_{M} S_{A} I_{M}}{N_{H}}-\xi E_{A}-\left(\sigma_{A}+\mu_{A}\right) E_{A}, \\
& \frac{d I_{A A}}{d t}=\alpha I_{A J}+\varepsilon_{A} \sigma_{A} E_{A}-\xi I_{A A}-\left(\gamma_{A A}+\mu_{A}\right) I_{A A}, \\
& \frac{d I_{S A}}{d t}=\alpha I_{S J}+\left(1-\varepsilon_{A}\right) \sigma_{A} E_{A}-\xi I_{S A}-\left(\gamma_{S A}+\mu_{A}\right) I_{S A}, \\
& \frac{d R_{A}}{d t}=\alpha R_{J}+\gamma_{A A} I_{A A}+\gamma_{S A} I_{S A}-\xi R_{A}-\mu_{A} R_{A}, \\
& \frac{d S_{S}}{d t}=\xi S_{A}-\frac{\beta_{S} b_{M} S_{S} I_{M}}{N_{H}}-\mu_{S} S_{S} \\
& \frac{d E_{S}}{d t}=\xi E_{A}+\frac{\beta_{S} b_{M} S_{S} I_{M}}{N_{H}}-\left(\sigma_{S}+\mu_{S}\right) E_{S}, \\
& \frac{d I_{A S}}{d t}=\xi I_{A A}+\varepsilon_{S} \sigma_{S} E_{S}-\left(\gamma_{A S}+\mu_{S}\right) I_{A S}
\end{aligned}
$$

$$
\begin{aligned}
& \frac{d I_{S S}}{d t}=\xi I_{S A}+\left(1-\varepsilon_{S}\right) \sigma_{S} E_{S}-\left(\gamma_{S S}+\mu_{S}\right) I_{S S}, \\
& \frac{d R_{S}}{d t}=\xi R_{A}+\gamma_{A S} I_{A S}+\gamma_{S S} I_{S S}-\mu_{S} R_{S}, \\
& \frac{d S_{M}}{d t} \\
& =\pi_{M} \\
& -\beta_{M} b_{M}\left[\frac{I_{A J}+I_{S J}+I_{A A}+I_{S A}+I_{A S}+I_{S S}}{N_{H}}\right] S_{M} \\
& -\mu_{M} S_{M} \\
& \frac{d E_{M}}{d t} \\
& =\beta_{M} b_{M}\left[\frac{I_{A J}+I_{S J}+I_{A A}+I_{S A}+I_{A S}+I_{S S}}{N_{H}}\right] S_{M} \\
& -\left(\sigma_{M}+\mu_{M}\right) E_{M}, \\
& \frac{d I_{M}}{d t}=\sigma_{M} E_{M}-\mu_{M} I_{M} .
\end{aligned}
$$

The flow diagram of the age-structured chikungunya model (10) is depicted in Figure 1 and the associated variables and parameters are described in Table 1. Model (10) is an extension of some of the chikungunya transmission models (e.g., those in [21, 25-30]) by (inter alia):

(a) Including a compartment for the exposed humans and mosquitoes (this was not considered in [21, 27, 28]).

(b) Adding a compartment for asymptomatic and symptomatic individuals (these were not considered in $[25,26,30])$.

(c) Including an age structure for humans (this was not included in $[26,29])$.

(d) Adding compartments for seniors (these were not included in $[21,25-28,30])$.

\section{Analysis of the Model}

\subsection{Basic Qualitative Properties}

3.1.1. Positivity and Boundedness of Solutions. For the agestructured chikungunya transmission model (10) to be epidemiologically meaningful, it is important to prove that all its state variables are nonnegative for all time. In other words, solutions of the model system (10) with non-negative initial data will remain non-negative for all time $t>0$.

Lemma 1. Let the initial data $F(0) \geq 0$, where $F(t)=$ $\left(S_{J}, E_{J}, I_{A J}, I_{S J}, R_{J}, S_{A}, E_{A}, I_{A A}, I_{S A}, R_{A}, S_{S}, E_{S}, I_{A S}, I_{S S}, R_{A}, S_{M}\right.$, $\left.E_{M}, I_{M}\right)$. Then the solutions $F(t)$ of the age-structured 
TABLE 1: Description of the variables and parameters of the agestructured chikungunya model (10).

\begin{tabular}{|c|c|}
\hline Variable & Description \\
\hline$S_{J}, S_{A}, S_{S}$ & $\begin{array}{l}\text { Population of susceptible juvenile, } \\
\text { adult, and senior humans }\end{array}$ \\
\hline$E_{J}, E_{A}, E_{S}$ & $\begin{array}{l}\text { Population of exposed juvenile, } \\
\text { adult, and senior humans }\end{array}$ \\
\hline$I_{A J}, I_{S J}$ & $\begin{array}{l}\text { Population of asymptomatic and } \\
\text { symptomatic juvenile humans }\end{array}$ \\
\hline$I_{A A}, I_{S A}$ & $\begin{array}{l}\text { Population of asymptomatic and } \\
\text { symptomatic adult humans }\end{array}$ \\
\hline$I_{A S}, I_{S S}$ & $\begin{array}{l}\text { Population of asymptomatic and } \\
\text { symptomatic senior humans }\end{array}$ \\
\hline$R_{J}, R_{A}, R_{S}$ & $\begin{array}{l}\text { Population of recovered juvenile, } \\
\text { adult, and senior humans }\end{array}$ \\
\hline$S_{M}$ & $\begin{array}{l}\text { Population of susceptible } \\
\text { mosquitoes }\end{array}$ \\
\hline$E_{M}$ & Population of exposed mosquitoes \\
\hline$I_{M}$ & Population of infectious mosquitoes \\
\hline Parameter & Description \\
\hline$\pi_{J}$ & $\begin{array}{l}\text { Recruitment rate of juvenile } \\
\text { humans }\end{array}$ \\
\hline$\pi_{M}$ & Recruitment rate of mosquitoes \\
\hline$\alpha, \xi$ & Juvenile and adult maturation rates \\
\hline$\beta_{J}, \beta_{A}, \beta_{S}$ & $\begin{array}{l}\text { Transmission probability per } \\
\text { contact for susceptible humans }\end{array}$ \\
\hline$\beta_{M}$ & $\begin{array}{l}\text { Transmission probability per } \\
\text { contact for susceptible mosquitoes }\end{array}$ \\
\hline$b_{M}$ & Mosquito biting rate \\
\hline$\mu_{J}, \mu_{A}, \mu_{S}$ & $\begin{array}{l}\text { Natural death rate of juvenile, adult, } \\
\text { and senior humans }\end{array}$ \\
\hline$\mu_{M}$ & Natural death rate of mosquitoes \\
\hline$\varepsilon_{J}, \varepsilon_{A}, \varepsilon_{S}$ & $\begin{array}{l}\text { Fraction of exposed humans } \\
\text { becoming asymptomatic and } \\
\text { symptomatic }\end{array}$ \\
\hline$\sigma_{J}, \sigma_{A}, \sigma_{S}$ & $\begin{array}{l}\text { Progression rate of exposed } \\
\text { juvenile, adult, and senior humans }\end{array}$ \\
\hline$\gamma_{A J}, \gamma_{S J}$ & $\begin{array}{l}\text { Recovery rate of asymptomatic and } \\
\text { symptomatic juvenile humans }\end{array}$ \\
\hline$\gamma_{A A}, \gamma_{S A}$ & $\begin{array}{l}\text { Recovery rate of asymptomatic and } \\
\text { symptomatic adult humans }\end{array}$ \\
\hline$\gamma_{A S}, \gamma_{S S}$ & $\begin{array}{l}\text { Recovery rate of asymptomatic and } \\
\text { symptomatic senior humans }\end{array}$ \\
\hline$\sigma_{M}$ & $\begin{array}{l}\text { Progression rate of exposed } \\
\text { mosquitoes }\end{array}$ \\
\hline
\end{tabular}

chikungunya model (10) are nonnegative for all $t>0$. Furthermore

$$
\begin{array}{r}
\limsup _{t \rightarrow \infty} N_{H}(t)=\frac{\pi_{J}}{\mu_{H}}, \\
\limsup _{t \rightarrow \infty} N_{M}(t)=\frac{\pi_{M}}{\mu_{M}}
\end{array}
$$

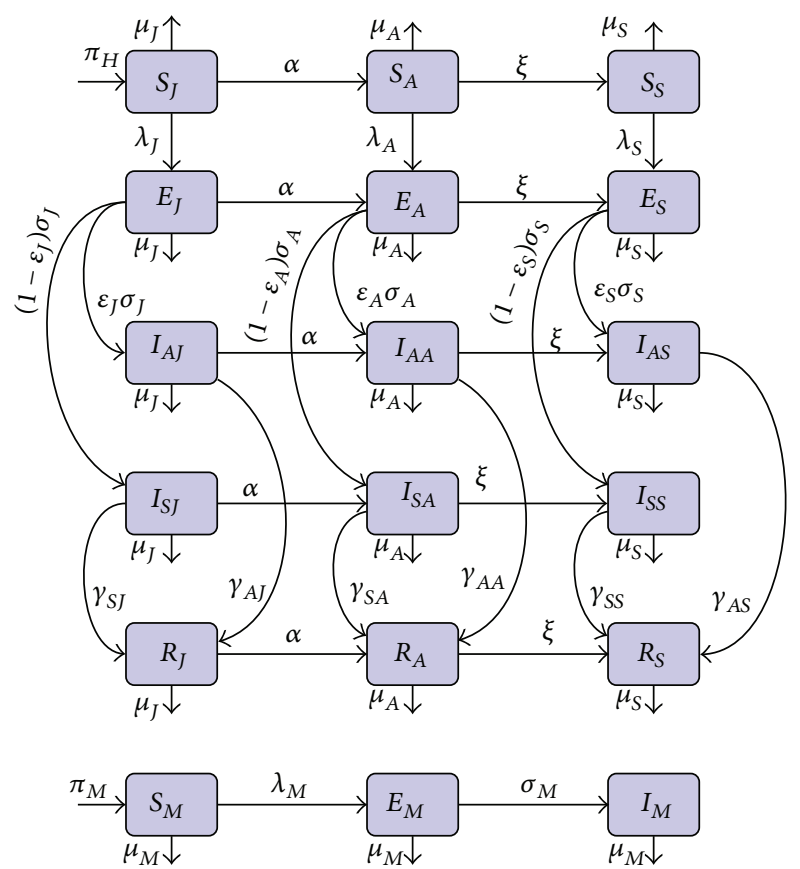

FIGURE 1: Systematic flow diagram of the age-structured chikungunya model (10).

with

$$
\begin{aligned}
N_{H}(t)= & S_{J}(t)+E_{J}(t)+I_{A J}(t)+I_{S J}(t)+R_{J}(t) \\
& +S_{A}(t)+E_{A}(t)+I_{A A}(t)+I_{S A}(t) \\
& +R_{A}(t)+S_{S}(t)+E_{S}(t)+I_{A S}(t) \\
& +I_{S S}(t)+R_{S}(t) \\
N_{M}(t)= & S_{M}(t)+E_{M}(t)+I_{M}(t) .
\end{aligned}
$$

The proof of Lemma 1 is given in Appendix B.

3.1.2. Invariant Regions. The age-structured chikungunya model (10) will be analyzed in a biologically feasible region as follows. Consider the feasible region

$$
\Omega=\Omega_{H} \times \Omega_{M} \subset \mathbb{R}_{+}^{15} \times \mathbb{R}_{+}^{3}
$$

with

$$
\begin{aligned}
& \Omega_{H}=\left\{S_{J}, E_{J}, I_{S J}, I_{A J}, R_{J}, S_{A}, E_{A}, I_{S A}, I_{A A}, R_{A}, S_{S}, E_{S}, I_{S S},\right. \\
& \left.I_{A S}, R_{S}: N_{H}(t) \leq \frac{\pi_{J}}{\mu_{H}}\right\}, \\
& \Omega_{M}=\left\{S_{M}, E_{M}, I_{M}: N_{M}(t) \leq \frac{\pi_{M}}{\mu_{M}}\right\} .
\end{aligned}
$$

Lemma 2. The region $\Omega \subset \mathbb{R}_{+}^{18}$ is positively invariant for the age-structured chikungunya model (10) with nonnegative initial conditions in $\mathbb{R}_{+}^{18}$. 
The proof of Lemma 2 is given in Appendix C.

In the next section, the conditions for the existence and stability of the equilibria of the age-structured chikungunya model (10) are explored.

3.2. Stability of Disease-Free Equilibrium (DFE). The agestructured chikungunya model (10) has a disease-free equilibrium (DFE), obtained by setting the right-hand sides of the equations in the model to zero, given by

$$
\begin{aligned}
& \mathscr{E}_{0}=\left(S_{J}^{*}, E_{J}^{*}, I_{A J}^{*}, I_{S J}^{*}, R_{J}^{*}, S_{A}^{*}, E_{A}^{*}, I_{A A}^{*}, I_{S A}^{*}, R_{A}^{*}, S_{S}^{*}, E_{S}^{*}, I_{A S}^{*},\right. \\
& \left.I_{S S}^{*}, R_{S}^{*}, S_{M}^{*}, E_{M}^{*}, I_{M}^{*}\right)=\left(\frac{\pi_{J}}{\alpha+\mu_{J}}, 0,0,0,0, \frac{\alpha S_{J}^{*}}{\xi+\mu_{A}}, 0,0,\right. \\
& \left.\quad 0,0, \frac{\xi S_{A}^{*}}{\mu_{S}}, 0,0,0,0, \frac{\pi_{M}}{\mu_{M}}, 0,0\right) .
\end{aligned}
$$

The linear stability of $\mathscr{E}_{0}$ can be established using the next generation operator method on system (10). Taking $E_{J}$, $I_{S J}, I_{A J}, E_{A}, I_{S A}, I_{A A}, E_{S}, I_{S S}, I_{A S}, E_{M}, I_{M}$ as the infected compartments and then using the notation in [42], the
Jacobian matrices $F$ and $V$ for the new infection terms and the remaining transfer terms are, respectively, given by

F

$$
=\left(\begin{array}{ccccccccccc}
0 & 0 & 0 & 0 & 0 & 0 & 0 & 0 & 0 & 0 & \Phi_{J} \\
0 & 0 & 0 & 0 & 0 & 0 & 0 & 0 & 0 & 0 & 0 \\
0 & 0 & 0 & 0 & 0 & 0 & 0 & 0 & 0 & 0 & 0 \\
0 & 0 & 0 & 0 & 0 & 0 & 0 & 0 & 0 & 0 & \Phi_{A} \\
0 & 0 & 0 & 0 & 0 & 0 & 0 & 0 & 0 & 0 & 0 \\
0 & 0 & 0 & 0 & 0 & 0 & 0 & 0 & 0 & 0 & 0 \\
0 & 0 & 0 & 0 & 0 & 0 & 0 & 0 & 0 & 0 & \Phi_{S} \\
0 & 0 & 0 & 0 & 0 & 0 & 0 & 0 & 0 & 0 & 0 \\
0 & 0 & 0 & 0 & 0 & 0 & 0 & 0 & 0 & 0 & 0 \\
0 & \Phi_{M} & \Phi_{M} & 0 & \Phi_{M} & \Phi_{M} & 0 & \Phi_{M} & \Phi_{M} & 0 & 0 \\
0 & 0 & 0 & 0 & 0 & 0 & 0 & 0 & 0 & 0 & 0
\end{array}\right),
$$

where $\Phi_{J}=\beta_{J} b_{M} \mu_{H} / k_{1}, \Phi_{A}=\beta_{A} b_{M} \alpha / k_{1}, \Phi_{S}=\beta_{S} b_{M} \xi \alpha /$ $\mu_{H} k_{1}, \Phi_{M}=\pi_{M} \beta_{M} b_{M} \mu_{H} / \mu_{M} \pi_{J}$, and

$$
V=\left(\begin{array}{ccccccccccc}
k_{2} & 0 & 0 & 0 & 0 & 0 & 0 & 0 & 0 & 0 & 0 \\
-\varepsilon_{J} \sigma_{J} & k_{3} & 0 & 0 & 0 & 0 & 0 & 0 & 0 & 0 & 0 \\
-\left(1-\varepsilon_{J}\right) \sigma_{J} & 0 & k_{4} & 0 & 0 & 0 & 0 & 0 & 0 & 0 & 0 \\
-\alpha & 0 & 0 & k_{7} & 0 & 0 & 0 & 0 & 0 & 0 & 0 \\
0 & -\alpha & 0 & -\varepsilon_{A} \sigma_{A} & k_{8} & 0 & 0 & 0 & 0 & 0 & 0 \\
0 & 0 & -\alpha-\left(1-\varepsilon_{A}\right) \sigma_{A} & 0 & k_{9} & 0 & 0 & 0 & 0 & 0 \\
0 & 0 & 0 & -\xi & 0 & 0 & k_{11} & 0 & 0 & 0 & 0 \\
0 & 0 & 0 & 0 & -\xi & 0 & -\varepsilon_{S} \sigma_{S} & k_{12} & 0 & 0 & 0 \\
0 & 0 & 0 & 0 & 0 & -\xi & -\left(1-\varepsilon_{S}\right) \sigma_{S} & 0 & k_{13} & 0 & 0 \\
0 & 0 & 0 & 0 & 0 & 0 & 0 & 0 & 0 & k_{14} & 0 \\
0 & 0 & 0 & 0 & 0 & 0 & 0 & 0 & 0 & -\sigma_{M} & \mu_{M}
\end{array}\right),
$$

where $k_{1}=\pi_{J}, k_{2}=\alpha+\sigma_{J}+\mu_{J}, k_{3}=\alpha+\gamma_{A J}+\mu_{J}, k_{4}=$ $\alpha+\gamma_{S J}+\mu_{J}, k_{7}=\xi+\sigma_{A}+\mu_{A}, k_{8}=\xi+\gamma_{A A}+\mu_{A}, k_{9}=\xi+\gamma_{S A}+\mu_{A}$, $k_{11}=\sigma_{S}+\mu_{S}, k_{12}=\gamma_{A S}+\mu_{S}, k_{13}=\gamma_{S S}+\mu_{S}$, and $k_{14}=\sigma_{M}+\mu_{M}$.

It follows that the reproduction number of the agestructured chikungunya model (10) is given by

$$
\mathscr{R}_{0}=\rho\left(F V^{-1}\right)=\sqrt{\left(\mathscr{R}_{J M}+\mathscr{R}_{A M}+\mathscr{R}_{S M}\right) \mathscr{R}_{M H}},
$$

where $\rho$ is the spectral radius and

$$
\begin{aligned}
& \mathscr{R}_{J M}=\beta_{J} b_{M} S_{J}^{*}\left[k _ { 1 1 } k _ { 1 2 } k _ { 1 3 } k _ { 7 } k _ { 8 } k _ { 9 } \sigma _ { J } \left[\left(1-\varepsilon_{J}\right) k_{3}\right.\right. \\
& \left.\quad+\varepsilon_{J} k_{4}\right] \\
& \quad+\alpha k_{11} k_{12} k_{13}\left(\sigma_{J} k_{7}\left[\left(1-\varepsilon_{J}\right) k_{3} k_{8}+\varepsilon_{J} k_{9} k_{4}\right]\right. \\
& \left.\quad+\sigma_{A} k_{4} k_{3}\left[\left(1-\varepsilon_{A}\right) k_{8}+\varepsilon_{A} k_{9}\right]\right)
\end{aligned}
$$

$$
\begin{aligned}
& +\xi \alpha\left\{\sigma_{J} k_{11} k_{7}\left[\left(1-\varepsilon_{J}\right) k_{3} k_{8} k_{12}+\varepsilon_{J} k_{4} k_{9} k_{13}\right]\right. \\
& +\sigma_{A} k_{3} k_{4} k_{11}\left[\left(1-\varepsilon_{A}\right) k_{8} k_{12}+\varepsilon_{A} k_{9} k_{13}\right] \\
& \left.\left.+\sigma_{S} k_{3} k_{4} k_{8} k_{9}\left[\left(1-\varepsilon_{S}\right) k_{12}+\varepsilon_{S} k_{13}\right]\right\}\right] \\
& \mathscr{R}_{A M}=\beta_{A} b_{M} S_{A}^{*} k_{2} k_{3} k_{4}\left[\sigma _ { A } k _ { 1 2 } k _ { 1 1 } k _ { 1 3 } \left[\left(1-\varepsilon_{A}\right) k_{8}\right.\right. \\
& \left.+\varepsilon_{A} k_{9}\right]+\xi\left\{\sigma_{A} k_{11}\left[\left(1-\varepsilon_{A}\right) k_{8} k_{12}+\varepsilon_{A} k_{9} k_{13}\right]\right. \\
& \left.\left.+\sigma_{S} k_{8} k_{9}\left[\left(1-\varepsilon_{S}\right) k_{12}+\varepsilon_{S} k_{13}\right]\right\}\right] \\
& \mathscr{R}_{S M}=\beta_{S} b_{M} \sigma_{S} S_{S}^{*} k_{2} k_{3} k_{4} k_{8} k_{7} k_{9}\left[\left(1-\varepsilon_{S}\right) k_{12}+k_{13} \varepsilon_{S}\right] \\
& \mathscr{R}_{M H}=\frac{\sigma_{M} \beta_{M} S_{M}^{*} \mu_{H}^{2} b_{M}}{\left(k_{2} k_{3} k_{4} k_{8} k_{7} k_{9} k_{11} k_{12} k_{13} k_{14} \mu_{M} \pi_{J}^{2}\right)} .
\end{aligned}
$$




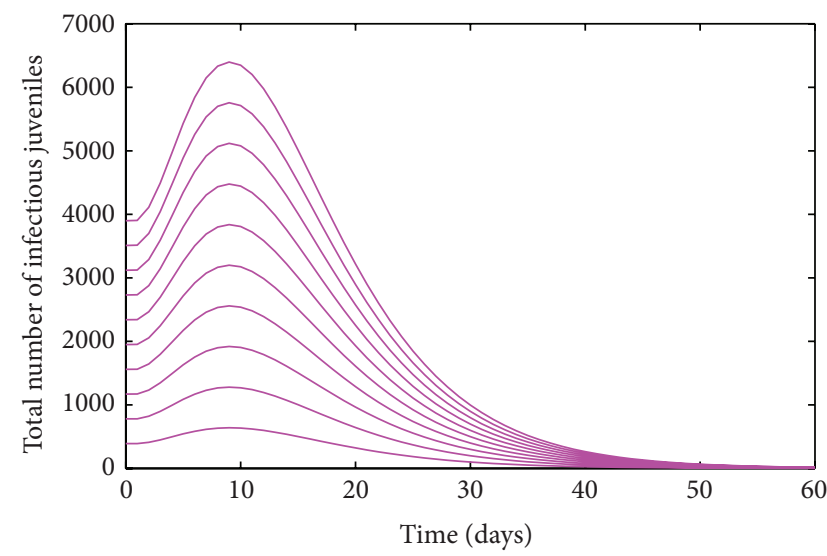

(a)

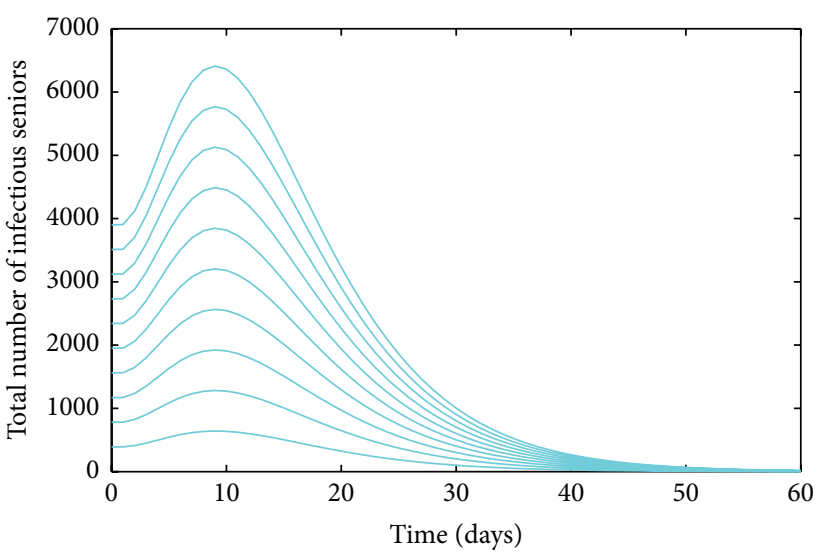

(c)

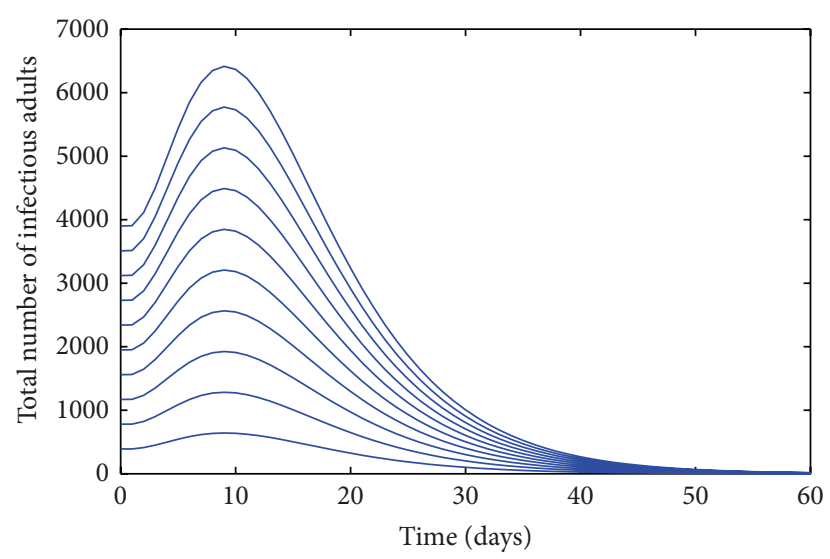

(b)

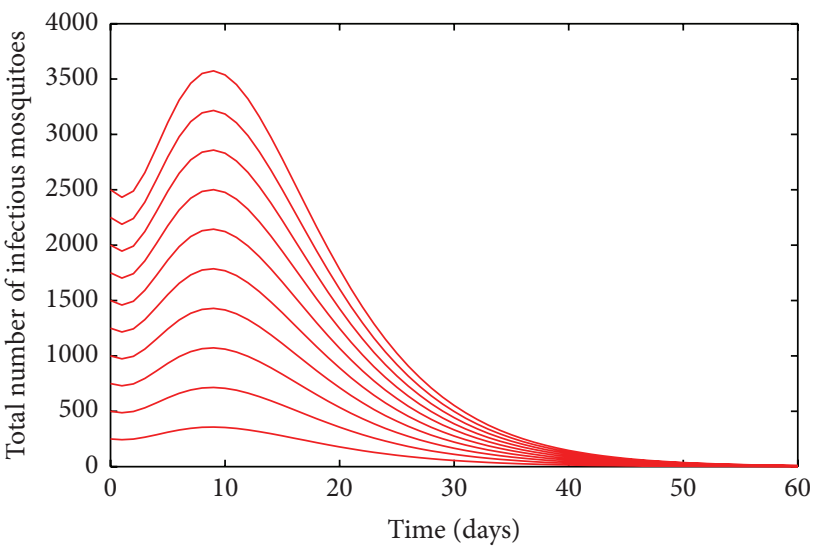

(d)

Figure 2: Simulation of the age-structured chikungunya model (10) as a function of time when $\mathscr{R}_{0}<1$. (a) Total number of infectious (asymptomatic and symptomatic) juveniles. (b) Total number of infectious (asymptomatic and symptomatic) adults. (c) Total number of infectious (asymptomatic and symptomatic) seniors. (d) Total number of infectious mosquitoes. Parameter values used are as given in Table 3.

Furthermore, the expression $\mathscr{R}_{I M}$ is the number of secondary infections in juveniles by one infectious mosquito, $\mathscr{R}_{A M}$ is the number of secondary infections in adults by one introduced infectious mosquito, $\mathscr{R}_{S M}$ is the number of secondary infections in seniors as a result of one infectious mosquito, and lastly $\mathscr{R}_{M H}$ is the number of secondary infections in mosquitoes resulting from a newly introduced infectious juvenile, adult, and senior. Further, using Theorem 2 in [42], the following result is established.

Lemma 3. The disease-free equilibrium (DFE) of the agestructured chikungunya model (10) is locally asymptotically stable (LAS) if $\mathscr{R}_{0}<1$ and unstable if $\mathscr{R}_{0}>1$.

The basic reproduction number $\mathscr{R}_{0}$ is defined as the average number of new infections that result from one infectious individual in a population that is fully susceptible [42-45]. The epidemiological significance of Lemma 3 is that chikungunya will be eliminated from the community if the reproduction number $\left(\mathscr{R}_{0}\right)$ can be brought to (and maintained at) a value less than unity. Figure 2 shows convergence of the solutions of the age-structured chikungunya model (10) to the DFE $\left(\mathscr{E}_{0}\right)$ for the case when $\mathscr{R}_{0}<1$ (in accordance with Lemma 3).

3.3. Global Asymptotic Stability of the DFE. Consider the feasible region

$$
\Omega_{1}=\left\{X \in \Omega: S_{J} \leq S_{J}^{*}, S_{A} \leq S_{A}^{*}, S_{S} \leq S_{S}^{*}, S_{M} \leq S_{M}^{*}\right\},
$$

where $X=S_{J}, E_{J}, I_{S J}, I_{A J}, R_{J}, S_{A}, E_{A}, I_{S A}, I_{A A}, R_{A}, S_{S}, E_{S}, I_{S S}$, $I_{A S}, R_{S}, S_{M}, E_{M}, I_{M}$.

Lemma 4. The region $\Omega_{1}$ is positively invariant for the agestructured chikungunya model (10).

The proof of Lemma 4 is given in Appendix D.

Theorem 5. The DFE, $\mathscr{E}_{0}$, of the age-structured chikungunya model (10), is globally asymptotically stable (GAS) in $\Omega_{1}$ whenever $\mathscr{R}_{0} \leq 1$.

The proof of Theorem 5 is given in Appendix E. 
3.4. Existence of Endemic Equilibrium Point (EEP). In this section, we will investigate conditions for the existence of endemic equilibria (i.e., equilibria where the infected components of the age-structured model (10) are nonzero).

Let

$$
\begin{gathered}
\mathscr{E}_{1}=\left(S_{J}^{* *}, E_{J}^{* *}, I_{A J}^{* *}, I_{S J}^{* *}, R_{J}^{* *}, S_{A}^{* *}, E_{A}^{* *}, I_{A A}^{* *}, I_{S A}^{* *}, R_{A}^{* *}, S_{S}^{* *},\right. \\
\left.E_{A}^{* *}, I_{A S}^{* *}, I_{S S}^{* *}, R_{S}^{* *}, S_{M}^{* *}, E_{M}^{* *}, I_{M}^{* *}\right)
\end{gathered}
$$

be an arbitrary endemic equilibrium of age-structured chikungunya model (10). Also, let

$$
\lambda_{J}^{* *}=\frac{\beta_{J} b_{M} I_{M}^{* *}}{N_{H}^{* *}}
$$

$$
\begin{aligned}
& \lambda_{A}^{* *}=\frac{\beta_{A} b_{M} I_{M}^{* *}}{N_{H}^{* *}}, \\
& \lambda_{S}^{* *}=\frac{\beta_{S} b_{M} I_{M}^{* *}}{N_{H}^{* *}}, \\
& \lambda_{M}^{* *}=\beta_{M} b_{M}\left[\frac{I_{A J}^{* *}+I_{S J}^{* *}+I_{A A}^{* *}+I_{S A}^{* *}+I_{A S}^{* *}+I_{S S}^{* *}}{N_{H}^{* *}}\right]
\end{aligned}
$$

be the forces of infection for susceptible juveniles, adults, and seniors and susceptible mosquitoes at steady state, respectively. Components of the steady-state solution of the equations of the age-structured chikungunya model (10) are given in Appendix F. Substituting the expressions for $I_{A J}^{* *}, I_{S J}^{* *}$, $I_{A A}^{* *}, I_{S A}^{* *}, I_{A S}^{* *}$ and $I_{S S}^{* *}$ into (22) for $\lambda_{M}^{* *}$ and simplifying gives

$$
\lambda_{M}^{* *}=\frac{b_{M}^{2} \beta_{M} I_{M}^{* *} \mu_{H}\left[a_{2}\left(I_{M}^{* *}\right)^{2}+a_{1} I_{M}^{* *}+a_{0}\right]}{k_{2} k_{3} k_{4} k_{7} k_{8} k_{9} k_{11} k_{12}\left(\beta_{J} b_{M} I_{M}^{* *} \mu_{H}+k_{1} \pi_{J}\right)\left(\beta_{A} b_{M} I_{M}^{* *} \mu_{H}+\pi_{J} k_{6}\right)\left(\beta_{S} b_{M} I_{M}^{* *} \mu_{H}+\pi_{J} \mu_{S}\right) k_{13}},
$$

where

$$
\begin{aligned}
a_{2} & =\beta_{J} \beta_{A} \beta_{S} b_{M}^{2} \mu_{H}^{2}\left\{k _ { 1 2 } k _ { 1 1 } k _ { 1 3 } \left\{\sigma _ { J } k _ { 9 } k _ { 8 } k _ { 7 } \left[\varepsilon_{J} k_{4}\right.\right.\right. \\
& \left.+\left(1-\varepsilon_{J}\right) k_{3}\right]+\alpha\left(\sigma_{J} k_{7}\left[\varepsilon_{J} k_{4} k_{9}+\left(1-\varepsilon_{J}\right) k_{8} k_{3}\right]\right. \\
& \left.\left.+\sigma_{A} k_{4} k_{3}\left[\varepsilon_{A} k_{9}+k_{8}\left(1-\varepsilon_{A}\right)\right]\right)\right\} \\
& +\alpha \xi\left(\sigma_{J} k_{11} k_{7}\left[\varepsilon_{J} k_{13} k_{9} k_{4}+\left(1-\varepsilon_{J}\right) k_{12} k_{8} k_{3}\right]\right. \\
& +\sigma_{A} k_{11} k_{4} k_{3}\left[k_{9} k_{13} \varepsilon_{A}+\left(1-\varepsilon_{A}\right) k_{12} k_{8}\right] \\
& \left.\left.+\sigma_{S} k_{9} k_{8} k_{4}\left[\varepsilon_{S} k_{13}+\left(1-\varepsilon_{S}\right) k_{12}\right]\right)\right\} \\
a_{1} & =\pi_{J} b_{H} \mu_{H}\left\{\sigma_{J} \beta_{J} k_{13} k_{12} k_{11} k_{9} k_{8} k_{7}\left(k_{6} \beta_{S}+\mu_{S} \beta_{A}\right)\right. \\
& +\left[\varepsilon_{J} k_{4}+\left(1-\varepsilon_{J}\right) k_{3}\right]+k_{13} k_{12} k_{11} \alpha \beta_{J}\left(\beta_{A} \mu_{S}\right. \\
& \left.+k_{6} \beta_{S}\right)\left\{k_{7}\left[\varepsilon_{J} k_{4} k_{9}+k_{3} k_{8}\left(1-\varepsilon_{J}\right)\right] \sigma_{J}\right. \\
& \left.+k_{4} k_{3}\left[\varepsilon_{A} k_{9}+\left(1-\varepsilon_{A}\right) k_{8}\right] \sigma_{A}\right\} \\
& +\alpha \beta_{A} \beta_{S} k_{13} k_{12} k_{11} k_{4} k_{3} k_{2} \sigma_{A}\left[\varepsilon_{A} k_{9}+\left(1-\varepsilon_{A}\right) k_{8}\right] \\
& +\alpha \xi \beta_{A} \beta_{S} k_{4} k_{3} k_{2}\left\{\sigma _ { A } k _ { 1 1 } \left[\varepsilon_{A} k_{13} k_{9}\right.\right. \\
& \left.\left.+\left(1-\varepsilon_{A}\right) k_{8} k_{12}\right]+\sigma_{S} k_{9} k_{8}\left[\varepsilon_{S} k_{13}+\left(1-\varepsilon_{S}\right) k_{12}\right]\right\} \\
& +\alpha \xi \beta_{J}\left(\beta_{A} \mu_{S}+\beta_{S} k_{6}\right)\left(\sigma _ { J } k _ { 1 1 } k _ { 7 } \left[\varepsilon_{J} k_{13} k_{9} k_{4}\right.\right. \\
& \left.+\left(1-\varepsilon_{J}\right) k_{12} k_{8} k_{3}\right]+\sigma_{A} k_{11} k_{4} k_{3}\left[\varepsilon_{A} k_{13} k_{9}\right. \\
& \left.+\left(1-\varepsilon_{A}\right) k_{12} k_{8}\right]+\sigma_{S} k_{9} k_{8} k_{4} k_{3}\left[\varepsilon_{S} k_{13}\right. \\
a_{0} & =\pi_{J}^{2}\left\{\beta _ { J } \sigma _ { J } \mu _ { S } k _ { 1 3 } k _ { 1 2 } k _ { 1 1 } k _ { 9 } k _ { 8 } k _ { 7 } k _ { 6 } \left[\varepsilon_{J} k_{4}+\left(1-\varepsilon_{J}\right)\right.\right. \\
& +\alpha \beta_{J} \mu_{S} k_{13} k_{12} k_{11} k_{6}\left(\sigma _ { J } k _ { 7 } \left[k_{9} k_{4} \varepsilon_{K}\right.\right. \\
& +1-\varepsilon_{12}
\end{aligned}
$$

$$
\begin{aligned}
& \left.\left.+\left(1-\varepsilon_{J}\right) k_{8} k_{3}\right]+\sigma_{A} k_{4} k_{3}\left[\varepsilon_{A}+\left(1-\varepsilon_{A}\right) k_{9} k_{8}\right]\right) \\
& +\alpha \beta_{A} \mu_{S} k_{13} k_{12} k_{11} k_{4} k_{3} k_{2} \sigma_{A}\left[\varepsilon_{A} k_{9}+\left(1-\varepsilon_{A}\right) k_{8}\right] \\
& +\xi \alpha \beta_{J} \mu_{S} k_{6}\left(\sigma _ { J } k _ { 1 1 } k _ { 7 } \left[\varepsilon_{J} k_{13} k_{9} k_{4}\right.\right. \\
& \left.+\left(1-\varepsilon_{J}\right) k_{12} k_{8} k_{3}\right]+\sigma_{A} k_{11} k_{4} k_{3}\left[\varepsilon_{A} k_{13} k_{9}\right. \\
& \left.+\left(1-\varepsilon_{A}\right) k_{12} k_{8}\right]+k_{9} k_{8} k_{4} k_{3}\left[\varepsilon_{S} k_{13}+\left(1-\varepsilon_{S}\right) k_{12}\right] \\
& \left.\cdot \sigma_{S}\right)+\xi \alpha \beta_{A} \mu_{S} k_{4} k_{3} k_{2}\left(\sigma _ { A } k _ { 1 1 } \left[\varepsilon_{A} k_{13} k_{9}\right.\right. \\
& \left.\left.+\left(1-\varepsilon_{A}\right) k_{8} k_{12}\right]+\sigma_{S} k_{9} k_{8}\left[\varepsilon_{S} k_{13}+\left(1-\varepsilon_{S}\right) k_{12}\right]\right) \\
& \left.+\xi \alpha \beta_{S} k_{4} k_{9} \sigma_{S} k_{8} k_{7} k_{3} k_{2}\left[\varepsilon_{S} k_{13}+\left(1-\varepsilon_{J}\right) k_{12}\right]\right\} .
\end{aligned}
$$

Substituting expression for $I_{M}^{* *}$ into the force of infection $\lambda_{J}^{* *}$ in (22) gives

$$
\lambda_{J}^{* *}=\frac{\beta_{J} b_{M} \sigma_{M} \lambda_{M}^{* *} \pi_{M} \mu_{H}}{k_{14} \mu_{M} \pi_{J}\left(\lambda_{M}^{* *}+\mu_{M}\right)}
$$

and then solving for $\lambda_{M}^{* *}$ gives

$$
\lambda_{M}^{* *}=-\frac{\lambda_{J}^{* *} k_{14} \mu_{M}^{2} \pi_{J}}{\left(\lambda_{J}^{* *} k_{14} \mu_{M} \pi_{J}-\beta_{J} b_{M} \sigma_{M} \pi_{M} \mu_{H}\right)}
$$

Substituting this result into (23), and simplifying, leads to the following cubic equation:

$$
b_{3}\left(\lambda_{J}^{* *}\right)^{3}+b_{2}\left(\lambda_{J}^{* *}\right)^{2}+b_{1} \lambda_{J}^{* *}+b_{0}\left(1-\mathscr{R}_{0}^{2}\right),
$$


TABLE 2: Number of possible positive real roots of $f(x)$ for $\mathscr{R}_{0}>1$.

\begin{tabular}{lccccccc}
\hline Cases & $c_{3}$ & $c_{2}$ & $c_{1}$ & $c_{0}$ & $\mathscr{R}_{0}$ & Number of sign changes & Number of possible positive real roots (endemic equilibrium) \\
\hline 1 & + & + & + & - & $\mathscr{R}_{0}>1$ & 1 & 1 \\
2 & + & - & - & - & $\mathscr{R}_{0}>1$ & 1 & 1 \\
3 & + & + & - & - & $\mathscr{R}_{0}>1$ & 1 & 1 \\
\hline
\end{tabular}

where

$$
\begin{aligned}
b_{3} & \\
= & \pi_{J}^{4} \beta_{J} \beta_{A} \beta_{S} b_{M} \mu_{H} \mu_{M}^{2} k_{2} k_{3} k_{4} k_{8} k_{7} k_{9} k_{11} k_{12} k_{13} k_{14} \\
& +\pi_{J}^{3} \beta_{M} \mu_{M} k_{14} a_{2}, \\
b_{2} & \\
= & \pi_{J}^{4} \beta_{J} \beta_{A} \beta_{S} b_{M} \mu_{H} \mu_{M}^{2} k_{1} k_{2} k_{3} k_{4} k_{8} k_{7} k_{9} k_{11} k_{12} k_{13} k_{14} \\
& -\pi_{J}^{2} \beta_{J} \pi_{M} \beta_{M} b_{M} \sigma_{M} \mu_{H} a_{2} \\
& +\pi_{J}^{2} \beta_{J} \mu_{H} \beta_{M} \mu_{M} b_{M} k_{14} a_{1} \\
& +\pi_{J}^{4} \beta_{J}^{2} \beta_{A} b_{M} \mu_{S} \mu_{H}^{2} \mu_{M}^{2} k_{2} k_{3} k_{4} k_{8} k_{7} k_{9} k_{12} k_{11} k_{13} k_{14} \\
& +\pi_{J}^{4} \beta_{J}^{2} \beta_{S} \mu_{H} b_{M} \mu_{M}^{2} k_{2} k_{3} k_{4} k_{6} k_{7} k_{8} k_{9} k_{11} k_{12} k_{13} k_{14},
\end{aligned}
$$$$
b_{1}
$$

$$
\begin{aligned}
= & \pi_{J} \beta_{J}^{2} \mu_{H}^{2} \beta_{M} b_{M}^{2} \mu_{M} k_{14} a_{0} \\
& +\pi_{J}^{4} \beta_{J}^{2} \beta_{A} b_{M} \mu_{S} \mu_{H}^{2} \mu_{M}^{2} k_{1} k_{2} k_{3} k_{4} k_{8} k_{7} k_{9} k_{11} k_{12} k_{13} k_{14} \\
& -\pi_{J} \beta_{J}^{2} b_{M}^{2} \pi_{M} \beta_{M} \sigma_{M} \mu_{H}^{2} a_{1} \\
& +\pi_{J}^{4} \beta_{J}^{3} b_{M} \mu_{S} \mu_{H}^{2} \mu_{M}^{2} k_{2} k_{3} k_{4} k_{6} k_{7} k_{8} k_{9} k_{11} k_{12} k_{13} k_{14} \\
& +\pi_{J}^{4} \beta_{J}^{2} \beta_{S} b_{M} \mu_{H} \mu_{M}^{2} k_{1} k_{2} k_{3} k_{4} k_{6} k_{7} k_{8} k_{9} k_{11} k_{12} k_{13} k_{14}, \\
b_{0}= & \pi_{J}^{4} \beta_{J}^{3} b_{M} \mu_{H}^{2} \mu_{S} \mu_{M}^{2} k_{1} k_{2} k_{3} k_{4} k_{6} k_{8} k_{7} k_{9} k_{11} k_{12} k_{13} k_{14} .
\end{aligned}
$$

Thus, the number of possible positive real roots polynomial (27) can have depends on the signs of $b_{2}$ and $b_{1}$. This can be analyzed using the Descartes Rule of Signs on the cubic polynomial $f(x)=c_{3} x^{3}+c_{2} x^{2}+c_{1} x+c_{0}$, given in (27) (with $\left.x=\lambda_{J}^{* *}, c_{3}=b_{3}, c_{2}=b_{2}, c_{1}=b_{1}, c_{0}=b_{0}\left(1-\mathscr{R}_{0}^{2}\right)\right)$. The various possibilities for the roots of $f(x)$ are tabulated in Table 2 .

The following results (Theorem 6) follow from the various possible combinations for the roots of $f(x)$, in Table 2 .

Theorem 6. The age-structured chikungunya model (10) has a unique endemic equilibrium if $\mathscr{R}_{0}>1$.

Numerical simulations of the age-structured chikungunya model (10), depicted in Figure 3, show convergence to a unique endemic equilibrium when $\mathscr{R}_{0}>1$ (suggesting that the unique EEP of the age-structured chikungunya model (10) is asymptotically stable when it exists).

3.5. Backward Bifurcation Analysis: Special Case. Chikungunya rarely leads to death of the infected individuals $[1,2]$; however a number of deaths have been reported as a result of the infection [3, 5, 30, 46, 47]. We introduced into the asymptomatic and symptomatic human compartments disease-induced mortality parameters $\left(\delta_{A J}, \delta_{S J}, \delta_{A A}, \delta_{S A}, \delta_{A S}\right.$, $\left.\delta_{S S}\right)$ and study the implication on the dynamics of the disease transmission. Thus, the asymptomatic and symptomatic juveniles, adults, and seniors compartments of age-structured chikungunya model (10) can be written as follows:

$$
\begin{aligned}
\frac{d I_{A J}}{d t}= & \varepsilon_{J} \sigma_{J} E_{J}-\alpha I_{A J}-\left(\gamma_{A J}+\mu_{H}+\delta_{A J}\right) I_{A J}, \\
\frac{d I_{S J}}{d t}= & \left(1-\varepsilon_{J}\right) \sigma_{J} E_{J}-\alpha I_{S J}-\left(\gamma_{S J}+\mu_{H}+\delta_{S J}\right) I_{S J}, \\
\frac{d I_{A A}}{d t}= & \alpha I_{A J}+\varepsilon_{A} \sigma_{A} E_{A}-\xi I_{A A} \\
& -\left(\gamma_{A A}+\mu_{H}+\delta_{A A}\right) I_{A A}, \\
\frac{d I_{S A}}{d t}= & \alpha I_{S J}+\left(1-\varepsilon_{A}\right) \sigma_{A} E_{A}-\xi I_{S A} \\
& -\left(\gamma_{S A}+\mu_{H}+\delta_{S A}\right) I_{S A}, \\
\frac{d I_{A S}}{d t}= & \xi I_{A A}+\varepsilon_{S} \sigma_{S} E_{S}-\left(\gamma_{A S}+\mu_{H}+\delta_{A S}\right) I_{A S}, \\
\frac{d I_{S S}}{d t}= & \xi I_{S A}+\left(1-\varepsilon_{S}\right) \sigma_{S} E_{S}-\left(\gamma_{S S}+\mu_{H}+\delta_{S S}\right) I_{S S} .
\end{aligned}
$$

It can be shown that the reproduction number for the agestructured chikungunya model (10) with the asymptomatic and symptomatic human compartments stated in (29) is given by

$$
\begin{aligned}
\widetilde{\mathscr{R}}_{0} & =\left.\mathscr{R}_{0}\right|_{\delta_{A J}, \delta_{S J}, \delta_{A A}, \delta_{S A}, \delta_{A S}, \delta_{S S} \neq 0} \\
& =\sqrt{\left(\widetilde{\mathscr{R}}_{J M}+\widetilde{\mathscr{R}}_{A M}+\widetilde{\mathscr{R}}_{S M}\right) \widetilde{\mathscr{R}}_{M H}},
\end{aligned}
$$

where

$$
\begin{aligned}
& \widetilde{\mathscr{R}}_{J M}=\beta_{J} b_{M} S_{J}^{*}\left[k _ { 1 1 } k _ { 1 2 } k _ { 1 3 } \sigma _ { J } k _ { 7 } k _ { 8 } k _ { 9 } \left[\left(1-\varepsilon_{J}\right) k_{3}\right.\right. \\
& \left.\quad+\varepsilon_{J} k_{4}\right] \\
& +\alpha k_{11} k_{12} k_{13}\left(\sigma_{J} k_{7}\left[\left(1-\varepsilon_{J}\right) k_{3} k_{8}+\varepsilon_{J} k_{9} k_{4}\right]\right. \\
& \left.\quad+\sigma_{A} k_{4} k_{3}\left[\left(1-\varepsilon_{A}\right) k_{8}+\varepsilon_{A} k_{9}\right]\right) \\
& \quad+\xi \alpha\left\{\sigma_{J} k_{11} k_{7}\left[\left(1-\varepsilon_{J}\right) k_{3} k_{8} k_{12}+\varepsilon_{J} k_{4} k_{9} k_{13}\right]\right. \\
& \quad+\sigma_{A} k_{3} k_{4} k_{11}\left[\left(1-\varepsilon_{A}\right) k_{8} k_{12}+\varepsilon_{A} k_{9} k_{13}\right] \\
& \left.\left.+\sigma_{S} k_{3} k_{4} k_{8} k_{9}\left[\left(1-\varepsilon_{S}\right) k_{12}+\varepsilon_{S} k_{13}\right]\right\}\right],
\end{aligned}
$$




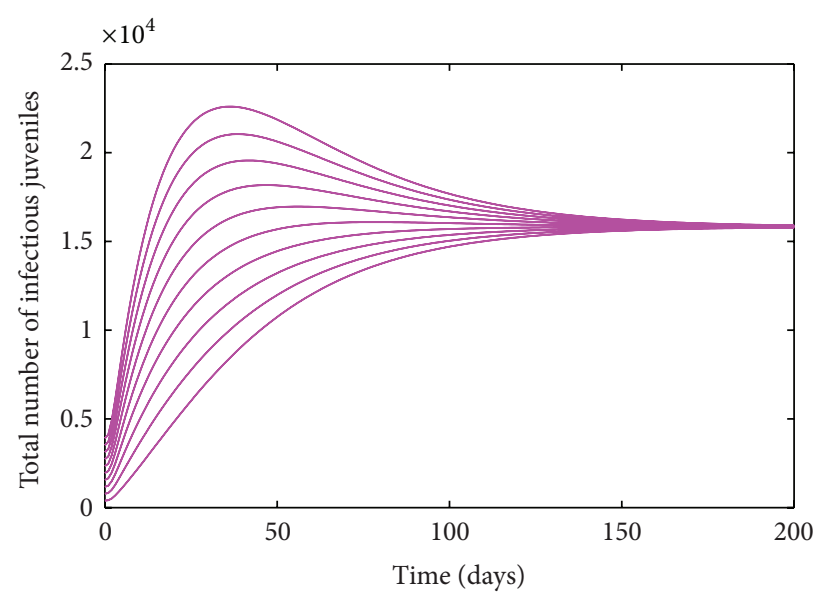

(a)

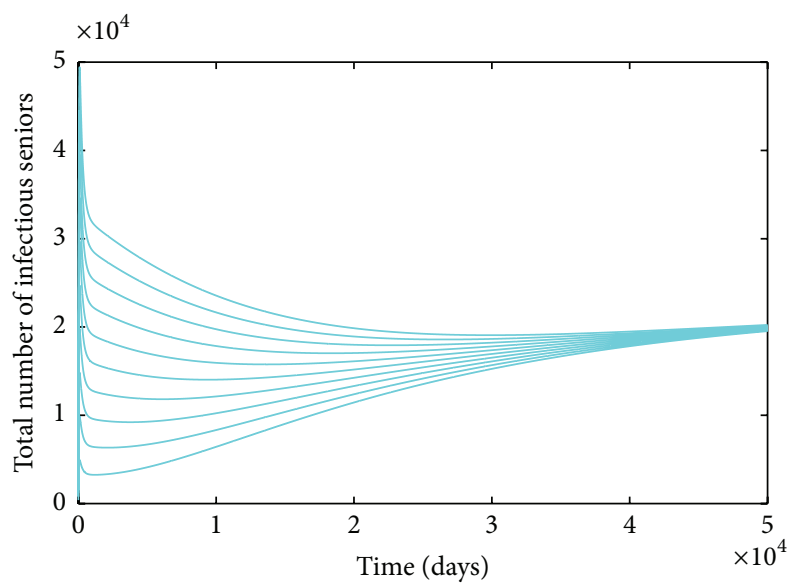

(c)

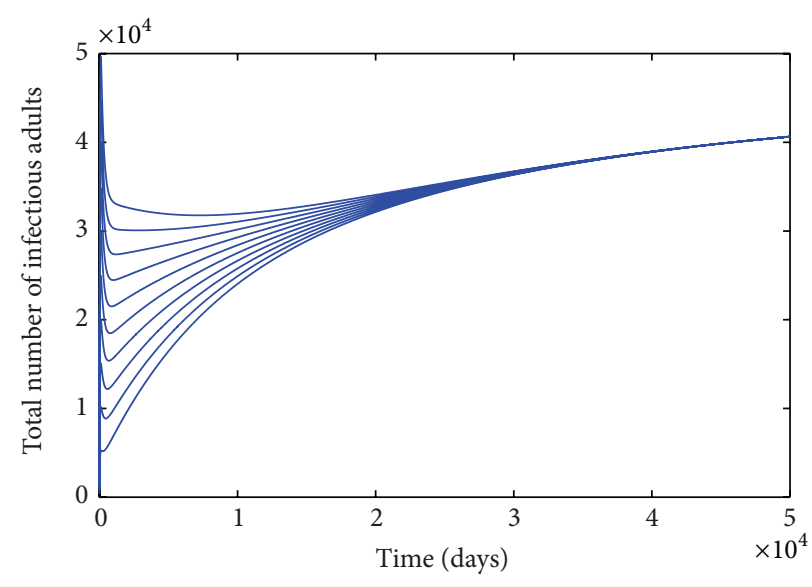

(b)

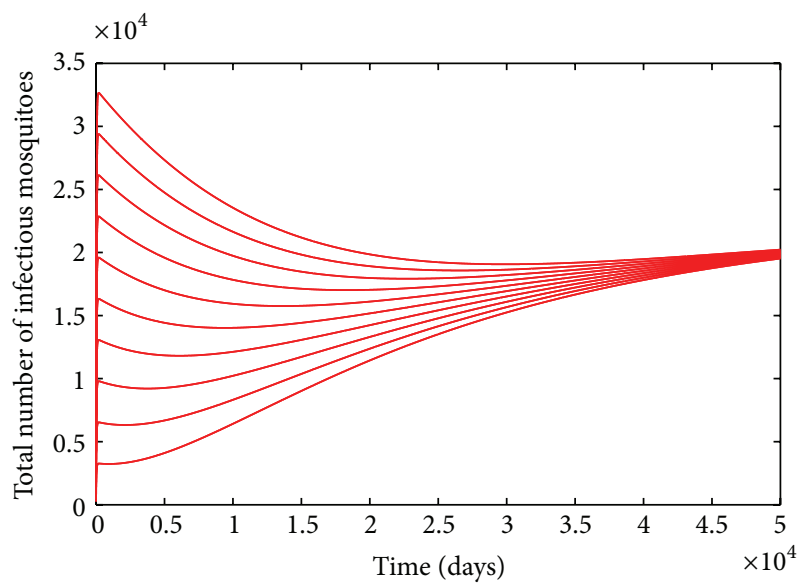

(d)

FIGURE 3: Simulation of the age-structured chikungunya model (10) as a function of time when $\mathscr{R}_{0}>1$. (a) Total number of infectious (asymptomatic and symptomatic) juveniles. (b) Total number of infectious (asymptomatic and symptomatic) adults. (c) Total number of infectious (asymptomatic and symptomatic) seniors. (d) Total number of infectious mosquitoes. Parameter values used are as given in Table 3.

$$
\begin{aligned}
& \widetilde{\mathscr{R}}_{A M}=\beta_{A} b_{M} S_{A}^{*} k_{2} k_{3} k_{4}\left[\sigma _ { A } k _ { 1 2 } k _ { 1 1 } k _ { 1 3 } \left[\left(1-\varepsilon_{A}\right) k_{8}\right.\right. \\
& \left.+\varepsilon_{A} k_{9}\right]+\xi\left\{\sigma_{A} k_{11}\left[\left(1-\varepsilon_{A}\right) k_{8} k_{12}+\varepsilon_{A} k_{9} k_{13}\right]\right. \\
& \left.\left.+\sigma_{S} k_{8} k_{9}\left[\left(1-\varepsilon_{S}\right) k_{12}+\varepsilon_{S} k_{13}\right]\right\}\right], \\
& \widetilde{\mathscr{R}}_{S M}=\beta_{S} b_{M} \sigma_{S} S_{S}^{*} k_{2} k_{3} k_{4} k_{8} k_{7} k_{9}\left[\left(1-\varepsilon_{S}\right) k_{12}+k_{13} \varepsilon_{S}\right], \\
& \widetilde{\mathscr{R}}_{M H}=\frac{\sigma_{M} \beta_{M} S_{M}^{*} \mu_{H}^{2} b_{M}}{\left(k_{2} k_{3} k_{4} k_{8} k_{7} k_{9} k_{11} k_{12} k_{13} k_{14} \mu_{M} \pi_{J}^{2}\right)},
\end{aligned}
$$

with $k_{3}=\alpha+\gamma_{A J}+\mu_{J}+\delta_{S J}, k_{4}=\alpha+\gamma_{S J}+\mu_{J}+\delta_{S J}, k_{8}=$ $\xi+\gamma_{A A}+\mu_{A}+\delta_{A A}, k_{9}=\xi+\gamma_{S A}+\mu_{A}+\delta_{S A}, k_{12}=\gamma_{A S}+\mu_{S}+\delta_{A S}$, and $k_{13}=\gamma_{S A}+\mu_{S}+\delta_{S A}$.

Models of disease transmission typically undergo a simple transcritical bifurcation (exchange of stability from the DFE to an endemic equilibrium) at $\widetilde{\mathscr{R}}_{0}=1$. The age-structured chikungunya model (10) with the asymptomatic and symptomatic human compartments stated in (29) is investigated for the possibility of the phenomenon of backward bifurcation (where a stable DFE coexists with a stable endemic equilibrium when the reproduction number, $\widetilde{\mathscr{R}}_{0}$, is less than unity) [48-57]. The epidemiological implication of backward bifurcation is that the effective control (or elimination) of chikungunya virus in the system is dependent on the initial sizes of the subpopulations. The possibility of the phenomenon of backward bifurcation in the age-structured chikungunya model (10) with the asymptomatic and symptomatic human compartments stated in (29) is explored using the centre manifold theory [51], as described in [58, Theorem 4.1].

Theorem 7. The age-structured chikungunya model (10) with the asymptomatic and symptomatic human compartments stated in (29) undergoes backward bifurcation at $\widetilde{\mathscr{R}}_{0}=1$ whenever inequality (G.9), given in Appendix G, holds.

The proof of Theorem 7 is given in Appendix G. The backward bifurcation property of the age-structured chikungunya model (10) with the asymptomatic and symptomatic human 
TABLE 3: Parameters values of models (10) and (32).

\begin{tabular}{|c|c|c|c|}
\hline Parameter & Values & Range & References \\
\hline \multirow{2}{*}{$\pi_{J}, \pi_{H}$} & $400 \times$ & 1 & \\
\hline & $400 \times \overline{15 \times 365}$ & $400 \times \overline{15 \times 365}-400 \times \overline{12 \times 365}$ & {$[26,31]$} \\
\hline \multirow[t]{2}{*}{$\alpha$} & & 1 & Assumed \\
\hline & $\begin{array}{c}\overline{16 \times 365} \\
1\end{array}$ & $\begin{array}{cc}18 \times 365 & 15 \times 365 \\
1 & 1\end{array}$ & \multirow{2}{*}{ Estimated } \\
\hline$\xi$ & $\overline{50 \times 365}$ & $\overline{55 \times 365}-\overline{45 \times 365}$ & \\
\hline$\beta_{J}, \beta_{A}, \beta_{S}, \beta_{H}$ & 0.24 & $0.001-0.54$ & {$[25,26,29,32,33]$} \\
\hline$b_{M}$ & 0.25 & $0.19-0.39$ & {$[26,34]$} \\
\hline \multirow[t]{2}{*}{$\mu_{J}$} & 1 & 1 & \multirow{2}{*}{ Assumed } \\
\hline & $\overline{3 \times 365}$ & $\overline{5 \times 365}-\overline{1 \times 365}$ & \\
\hline \multirow[t]{2}{*}{$\mu_{A}$} & 1 & $1-1$ & \multirow{2}{*}{ Assumed } \\
\hline & $\begin{array}{c}\overline{40 \times 365} \\
1\end{array}$ & $\begin{array}{cc}60 \times 365 & 18 \times 365 \\
1 & 1\end{array}$ & \\
\hline$\mu_{S}$ & $\overline{70 \times 365}$ & $\overline{80 \times 365}-\overline{60 \times 365}$ & Assumed \\
\hline \multirow[t]{2}{*}{$\mu_{H}$} & 1 & $1-\frac{1}{1}$ & \multirow{2}{*}[26,31]{} \\
\hline & $\overline{70 \times 365}$ & $\overline{76 \times 365}-\overline{68 \times 365}$ & \\
\hline$\varepsilon_{J}, \varepsilon_{A}, \varepsilon_{S}, \varepsilon_{H}$ & 0.155 & $0.03-0.28$ & [17] \\
\hline \multirow[t]{2}{*}{$\sigma_{J}, \sigma_{S}$} & 1 & 1 & \multirow{2}{*}{ Assumed } \\
\hline & $\overline{2 \times 3}$ & $\overline{2 \times 4}-\overline{2 \times 2}$ & \\
\hline \multirow[t]{2}{*}{$\sigma_{A}, \sigma_{H}$} & 1 & $1 \quad 1$ & \multirow{2}{*}[5,26,32,35-37]{} \\
\hline & $\overline{3}$ & $\overline{4}-\overline{2}$ & \\
\hline \multirow{2}{*}{$\gamma_{A J}, \gamma_{S J}$} & 1 & 111 & \multirow{2}{*}{ Assumed } \\
\hline & $\overline{1.5 \times 4.5}$ & $\overline{1.5 \times 8}-\overline{1.5 \times 3}$ & \\
\hline \multirow[t]{2}{*}{$\gamma_{A A}, \gamma_{S A}$} & 1 & $1-1$ & \multirow{2}{*}[26,28,36,37]{} \\
\hline & $\overline{4.5}$ & $\overline{7}-\overline{3}$ & \\
\hline \multirow{2}{*}{$\gamma_{A S}, \gamma_{S S}$} & 1 & $1-1$ & \multirow{2}{*}{ Assumed } \\
\hline & $\overline{2.5 \times 4.5}$ & $\overline{2.5 \times 8}-\overline{2.5 \times 3}$ & \\
\hline$\gamma_{A H}, \gamma_{S H}$ & $\frac{1}{45}$ & $\frac{1}{7}-\frac{1}{3}$ & {$[26,28,36,37]$} \\
\hline$\pi_{M}$ & $\begin{array}{c}4.5 \\
500 \times 0.1675\end{array}$ & $500 \times 0.015-500 \times 0.32$ & {$[26,31,38,39]$} \\
\hline$\beta_{M}$ & 0.24 & $0.005-0.35$ & {$[25,29,33,40,41]$} \\
\hline \multirow[t]{2}{*}{$\sigma_{M}$} & 1 & 11 & \multirow{2}{*}[6,28,32,36]{} \\
\hline & $\overline{3.5}$ & $\overline{6}^{-\overline{2}}$ & \\
\hline \multirow[t]{2}{*}{$\mu_{M}$} & 1 & $1-1$ & \multirow{2}{*}[28,32,35-37]{} \\
\hline & $\overline{14}$ & $\overline{42}-\overline{14}$ & \\
\hline
\end{tabular}

compartments given in (29) is illustrated by simulating the model using a set of parameter values given in Table 3 (such that the bifurcation parameters, $a$ and $b$, given in Appendix G, take the values $a=0.001792$ and $b=0.09056$, resp.). The backward bifurcation phenomenon of the agestructured chikungunya model (10) with the asymptomatic and symptomatic human compartments stated in (29) makes the effective control of the chikungunya in the population difficult, since in this case, disease control when $\widetilde{\mathscr{R}}_{0}<1$ is dependent on the initial sizes of the subpopulations of the age-structured chikungunya model (10) with the asymptomatic and symptomatic human compartments stated in (29). This phenomenon is illustrated numerically in Figure 4.

\section{Effect of Age Structure}

Following the approach in [59], the effect of age structure on the dynamics of the age-structured chikungunya model (10) will now be investigated by comparing its dynamical behavior with those of an equivalent model with no age structure given by

$$
\begin{aligned}
& \frac{d S_{H}}{d t}=\pi_{H}-\frac{\beta_{H} b_{M} I_{M}}{N_{H}} S_{H}-\alpha S_{H}-\mu_{H} S_{H}, \\
& \frac{d E_{H}}{d t}=\frac{\beta_{H} b_{M} I_{M}}{N_{H}} S_{H}-\alpha E_{H}-\left(\sigma_{H}+\mu_{H}\right) E_{H}, \\
& \frac{d I_{A H}}{d t}=\varepsilon_{H} \sigma_{H} E_{H}-\alpha I_{A H}-\left(\gamma_{A H}+\mu_{H}\right) I_{A H}, \\
& \frac{d I_{S H}}{d t}=\left(1-\varepsilon_{H}\right) \sigma_{H} E_{H}-\alpha I_{S H}-\left(\gamma_{S H}+\mu_{H}\right) I_{S H}, \\
& \frac{d R_{H}}{d t}=\gamma_{A H} I_{A H}+\gamma_{S H} I_{S H}-\alpha R_{H}-\mu_{H} R_{H}, \\
& \frac{d S_{M}}{d t}=\pi_{M}-\beta_{M} b_{M}\left[\frac{I_{A H}+I_{S H}}{N_{H}}\right] S_{M}-\mu_{M} S_{M},
\end{aligned}
$$




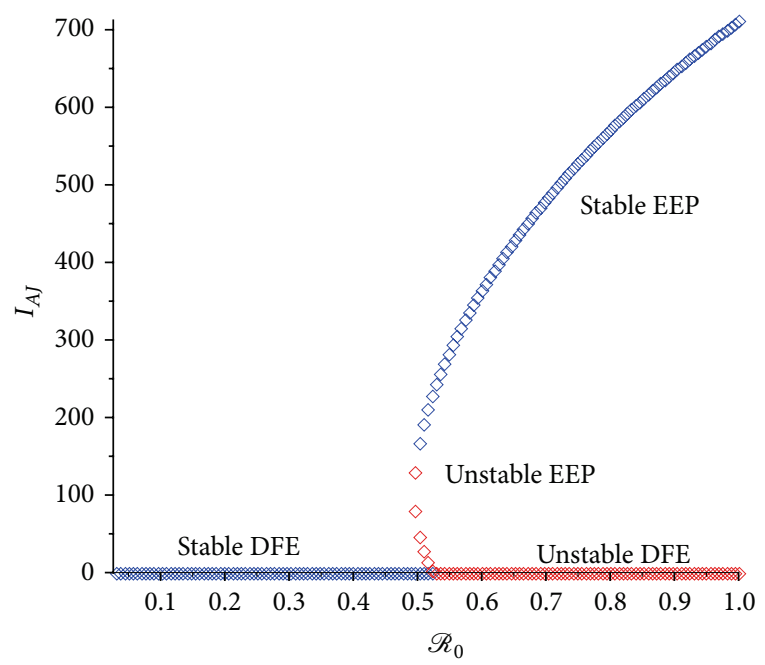

(a)

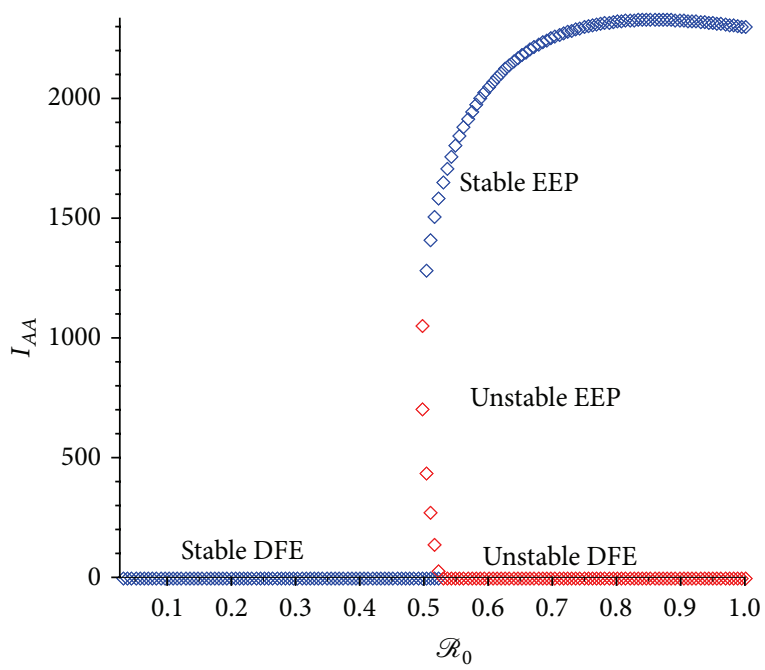

(c)

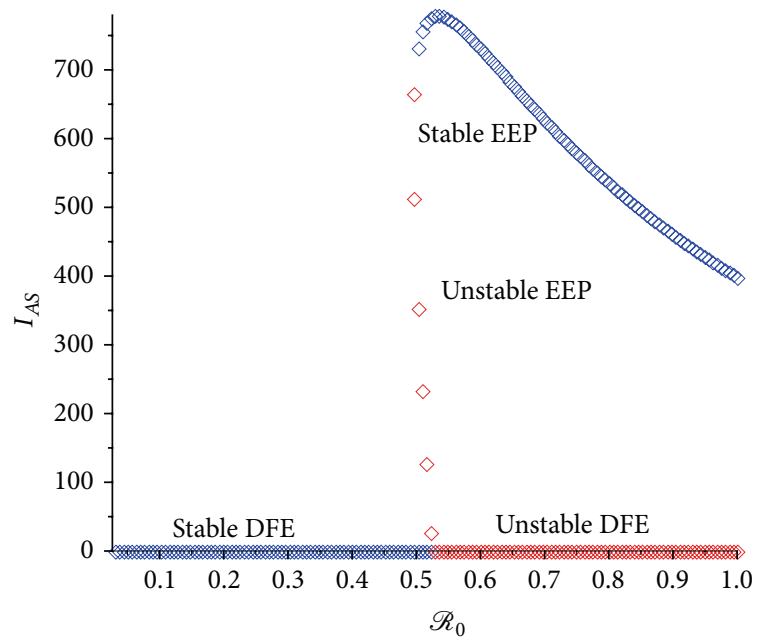

(e)

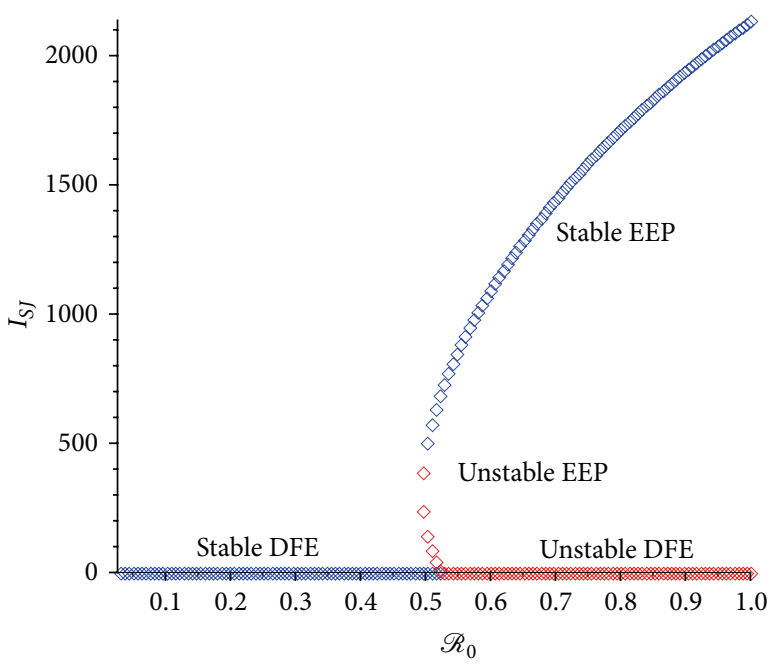

(b)

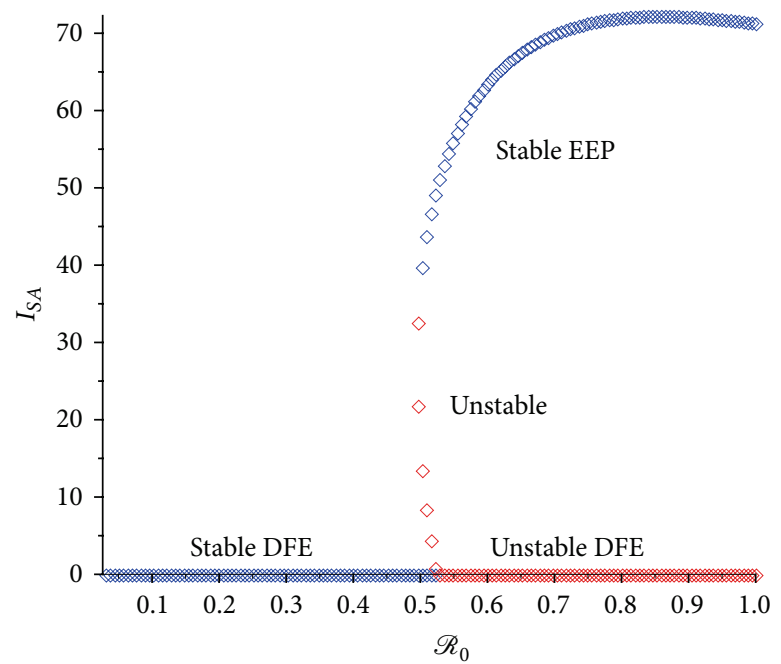

(d)

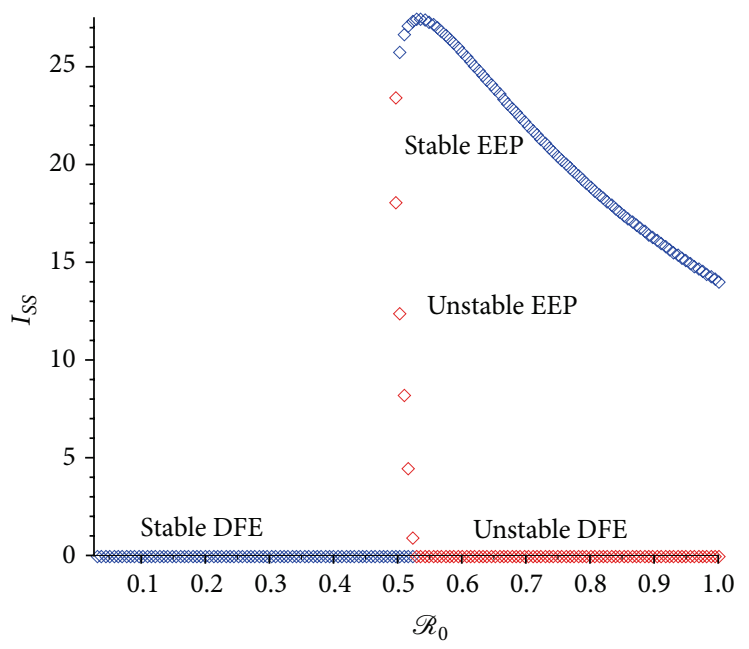

(f)

FIGURE 4: Backward bifurcation plot of the age-structured model (10) with the asymptomatic and symptomatic human compartments given in (29). (a) Asymptomatic juvenile; (b) symptomatic juvenile. (c) Asymptomatic adult; (d) symptomatic adult. (e) Asymptomatic senior; (f) symptomatic senior. Parameter values used are as given in Table 3. 


$$
\begin{aligned}
\frac{d E_{M}}{d t} & =\beta_{M} b_{M}\left[\frac{I_{A H}+I_{S H}}{N_{H}}\right] S_{M}-\left(\sigma_{M}+\mu_{M}\right) E_{M}, \\
\frac{d I_{M}}{d t} & =\sigma_{M} E_{M}-\mu_{M} I_{M},
\end{aligned}
$$

where $S_{H}=S_{J}+S_{A}+S_{S}, E_{H}=E_{J}+E_{A}+E_{S}, I_{A H}=I_{A J}+I_{A A}+I_{A S}$, $I_{S H}=I_{S J}+I_{S A}+I_{S S}, R_{H}=R_{J}+R_{A}+R_{S}$ and $\sigma_{H}=\sigma_{J}+\sigma_{A}+\sigma_{S}$, $\gamma_{A H}=\gamma_{A J}+\gamma_{A A}+\gamma_{A S}, \gamma_{S H}=\gamma_{S J}+\gamma_{S A}+\gamma_{S S}$, and $\alpha=\xi=0$.

The DFE of model (32) is given by

$$
\begin{aligned}
\mathscr{E}_{01} & =\left(S_{H}^{*}, E_{H}^{*}, I_{A H}^{*}, I_{S H}^{*}, R_{H}^{*}, S_{M}^{*}, E_{M}^{*}, I_{M}^{*}\right) \\
& =\left(\frac{\pi_{H}}{\mu_{H}}, 0,0,0,0, \frac{\pi_{M}}{\mu_{M}}, 0,0\right)
\end{aligned}
$$

and the associated reproduction number is

$$
\begin{aligned}
& \mathscr{R}_{H} \\
& :=\sqrt{\frac{\pi_{M} \beta_{M} \sigma_{M} \pi_{H} \beta_{H} b_{M}^{2}\left[g_{4} \varepsilon_{H} \sigma_{H}+g_{3}\left(1-\varepsilon_{H}\right) \sigma_{H}\right]}{g_{2} g_{3} g_{4} g_{6} \mu_{M}^{2} \mu_{H}}},
\end{aligned}
$$

where $g_{1}=\mu_{H}, g_{2}=\sigma_{H}+\mu_{H}, g_{3}=\gamma_{A H}+\mu_{H}, g_{4}=\gamma_{S H}+\mu_{H}$, $g_{5}=\mu_{H}$, and $g_{6}=\sigma_{M}+\mu_{M}$.

Using the approach in Section 3.3, we can show that the reduced model (32) has a DFE that is GAS if $\delta_{A H}=\delta_{S H}=0$, whenever $\mathscr{R}_{H}<1$; as such model (32) will not undergo backward bifurcation since the bifurcation coefficient, $a$, is given by

$$
a=\frac{2 b_{M} \mu_{H}\left(v_{2} w_{1} w_{8} \beta_{H}+v_{7} w_{3} w_{6} \beta_{M}+v_{7} w_{4} w_{6} \beta_{M}\right)}{\pi_{H}} ;
$$

using the expression in (38) below, (35) becomes

$$
a=-\frac{2 b_{M}^{5} \mu_{H}^{2} \sigma_{H}^{2} \pi_{M}^{2} \beta_{M}^{3} \sigma_{M}\left[\varepsilon_{H} g_{4}+\left(1-\varepsilon_{H}\right) g_{3}\right]^{2} \beta_{H}^{2}\left\{g_{3} g_{4} \mu_{M} g_{2}+b_{M} \beta_{M} \sigma_{H} g_{1}\left[\varepsilon_{H} g_{4}+\left(1-\varepsilon_{H}\right) g_{3}\right]\right\}}{\mu_{M}^{4} g_{6} \pi_{H}^{3} g_{3}^{3} g_{4}^{3} g_{2}^{3}}
$$

where $g_{1}=\mu_{H}, g_{2}=\sigma_{H}+\mu_{H}, g_{3}=\gamma_{A H}+\mu_{H}$, and $g_{4}=$ $\gamma_{S H}+\mu_{H}$.
However, if $\delta_{A H}, \delta_{S H} \neq 0$, then it follows that the reduced model (32) will undergo backward bifurcation if the coefficient $a$, in this case, given as

$$
a=\frac{-2 b_{M}\left[v_{2} w_{8} x_{1}\left(w_{2}+w_{3}+w_{5}\right) \beta_{H}+v_{7} w_{3} x_{6}\left(w_{3}+w_{1}+w_{2}+w_{5}\right) \beta_{M}-v_{7} w_{3} w_{6} x_{1} \beta_{M}\right]}{x_{1}^{2}}
$$

is positive, where

$$
\begin{aligned}
& w_{1}=\frac{-\beta_{H} b_{M} w_{8}}{g_{1} \mu_{M}}, \\
& w_{2}=\frac{\beta_{H} b_{M} w_{8}}{g_{2}}, \\
& w_{3}=\frac{\varepsilon_{H} \sigma_{H} w_{2}}{g_{3}}, \\
& w_{4}=\frac{\left(1-\varepsilon_{H}\right) \sigma_{H} w_{2}}{g_{4}}, \\
& w_{5}=\frac{\left(\gamma_{A H} w_{3}+\gamma_{S H} w_{4}\right)}{g_{5}}, \\
& w_{6}=-\frac{x_{6} \beta_{M} b_{M}\left(w_{3}+w_{4}\right)}{\mu_{M} x_{1}}, \\
& w_{7}=\frac{x_{6} \beta_{M} b_{M}\left(w_{3}+w_{4}\right)}{g_{6} x_{1}},
\end{aligned}
$$

$$
\begin{aligned}
& w_{8}>0, \\
& v_{1}=0, \\
& v_{5}=0, \\
& v_{6}=0, \\
& v_{2}=\frac{\left[\varepsilon_{H} \sigma_{H} v_{3}+\left(1-\varepsilon_{H}\right) \sigma_{H} v_{4}\right]}{g_{2}}, \\
& v_{3}=\frac{1}{g_{3}}\left(\gamma_{A H} v_{5}+\frac{x_{6} \beta_{M} b_{M}\left(v_{7}-v_{6}\right)}{x_{1}}\right), \\
& v_{4}=\frac{1}{g_{4}}\left(\gamma_{S H} v_{5}+\frac{x_{6} \beta_{M} b_{M}\left(v_{7}-v_{6}\right)}{x_{1}}\right), \\
& v_{7}=\frac{\sigma_{M} v_{8}}{g_{6}}, \\
& v_{8}=\frac{\left(\beta_{H} b_{M} v_{2}\right)}{\mu_{M}}
\end{aligned}
$$




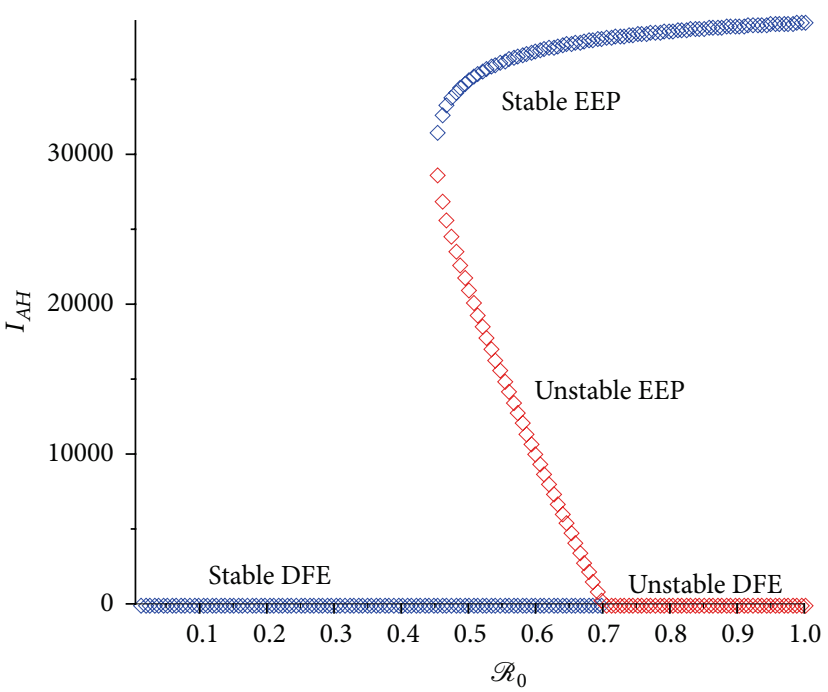

(a)

FIGURE 5: Backward bifurcation plot for without age-structured model (32). (a) Asymptomatic humans; (b) symptomatic humans. Parameter values used are as given in Table 3.

with $g_{1}=\mu_{H}, g_{2}=\sigma_{H}+\mu_{H}, g_{3}=\gamma_{A H}+\mu_{H}+\delta_{A H}, g_{4}=$ $\gamma_{S H}+\mu_{H}+\delta_{S H}, g_{5}=\mu_{H}$, and

$$
b=\frac{v_{7} x_{6} b_{M} w_{3}}{x_{1}}>0 .
$$

The phenomenon of backward bifurcation for model (32) without age structure is illustrated numerically in Figure 5.

Hence, the preceding analysis shows that the agestructured chikungunya model (10) and model (32) without age structure have the same qualitative dynamics with respect to the local and global asymptotic stability of the associated disease-free equilibrium (DFE) and the phenomenon of backward bifurcation.

Next, we compare the dynamics of the endemic equilibrium points of models (10) and (32). Let

$$
\mathscr{E}_{1}=\left(S_{H}^{* *}, E_{H}^{* *}, I_{S H}^{* *}, I_{A H}^{* *}, R_{H}^{* *}, S_{M}^{* *}, E_{M}^{* *}, I_{M}^{* *}\right)
$$

be an arbitrary endemic equilibrium of the reduced model (29) and let

$$
\begin{aligned}
& \lambda_{H}^{* *}=\frac{\beta_{H} b_{M} I_{M}^{* *}}{N_{H}^{* *}} \\
& \lambda_{M}^{* *}=\beta_{M} b_{M}\left[\frac{I_{A H}^{* *}+I_{S H}^{* *}}{N_{H}^{* *}}\right]
\end{aligned}
$$

be the forces infection for susceptible humans and susceptible mosquitoes at steady state, respectively. Solving the equations of the reduced model (32) at steady state gives

$$
\begin{aligned}
S_{H}^{* *} & =\frac{\pi_{H}}{\left(\lambda_{H}^{* *}+g_{1}\right)}, \\
E_{H}^{* *} & =\frac{\lambda_{H}^{* *} \pi_{H}}{g_{2}\left(\lambda_{H}^{* *}+g_{1}\right)},
\end{aligned}
$$

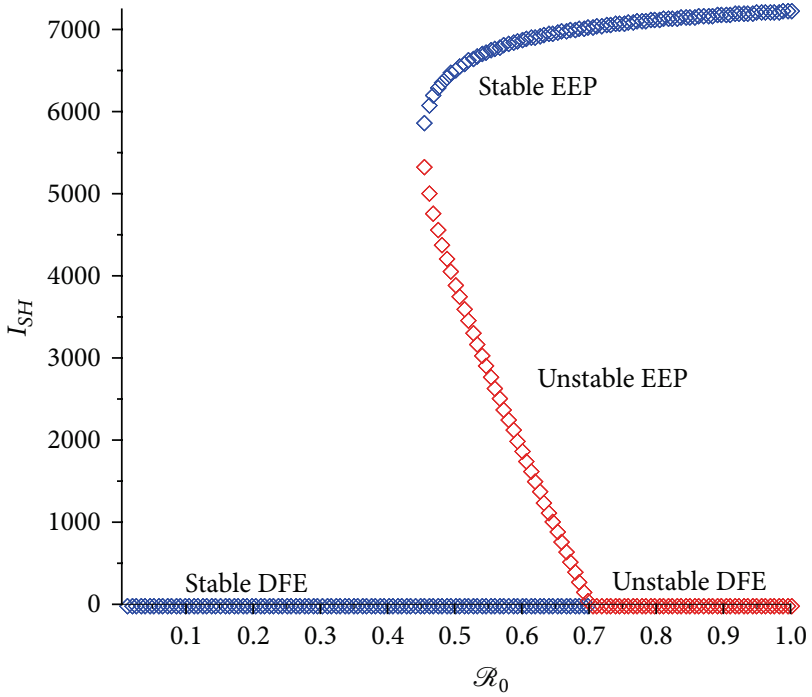

(b)

$$
\begin{aligned}
I_{A H}^{* *} & =\frac{\varepsilon_{H} \sigma_{H} \lambda_{H}^{* *} \pi_{H}}{g_{3} g_{2}\left(\lambda_{H}+g_{1}\right)}, \\
I_{S H}^{* *} & =\frac{\left(1-\varepsilon_{H}\right) \sigma_{H} \lambda_{H}^{* *} \pi_{H}}{g_{4} g_{2}\left(\lambda_{H}^{* *}+g_{1}\right)}, \\
R_{H}^{* *} & =\frac{\sigma_{H} \lambda_{H}^{* *} \pi_{H}\left[\gamma_{A H} \varepsilon_{H} g_{4}+\gamma_{S H}\left(1-\varepsilon_{H}\right) g_{3}\right]}{g_{5} g_{4} g_{2} g_{3}\left(\lambda_{H}^{* *}+g_{1}\right)}, \\
S_{M}^{* *} & =\frac{\pi_{M}}{\left(\lambda_{M}^{* *}+\mu_{M}\right)}, \\
E_{M}^{* *} & =\frac{\lambda_{M} \pi_{M}}{g_{6}\left(\lambda_{M}^{* *}+\mu_{M}\right)}, \\
I_{M}^{* *} & =\frac{\sigma_{M} \lambda_{M}^{* *} \pi_{M}}{g_{6} \mu_{M}\left(\lambda_{M}^{* *}+\mu_{M}\right)} .
\end{aligned}
$$

Substituting (42) into (41) shows that the positive endemic equilibrium of model (32) satisfies

$$
\begin{aligned}
& \lambda_{H}^{* *} \\
& =\frac{\pi_{H} g_{6} \mu_{M}^{2} g_{2} g_{3} g_{4} g_{1} \pi_{H}\left(\mathscr{R}_{H}^{2}-1\right)}{\pi_{H}^{2} g_{6} \mu_{M}\left\{g_{2} g_{3} g_{4} \mu_{M}+b_{M} \beta_{M} \mu_{H}\left[\varepsilon_{H} \sigma_{H} g_{4}+\left(1-\varepsilon_{H}\right) \sigma_{H} g_{3}\right]\right\}},
\end{aligned}
$$

where $\mathscr{R}_{H}=\left.\widetilde{\mathscr{R}}_{H}\right|_{\delta_{A H}, \delta_{S H} \neq 0}$. Hence, model (32) has a unique endemic equilibrium (obtained by substituting (42) into (43)) whenever $\widetilde{R}_{H}>1$.

4.1. Sensitivity Analysis. Sensitivity analysis [60-62] is carried out, on the parameters of the age-structured chikungunya model (10), to determine which of the parameters have the most significant impact on the outcome of the numerical 
TABLE 4: Initial conditions used in the simulations of model (10) with age structure.

\begin{tabular}{|c|c|c|c|c|}
\hline$S_{J}(0)=1130$ & $E_{J}(0)=123$ & $I_{A J}(0)=84$ & $I_{S J}(0)=211$ & $R_{J}(0)=0$ \\
\hline$S_{A}(0)=2196$ & $E_{A}(0)=264$ & $I_{A A}(0)=351$ & $I_{S A}(0)=1404$ & $R_{A}(0)=0$ \\
\hline$S_{S}(0)=300$ & $E_{S}(0)=224$ & $I_{A S}(0)=75$ & $I_{S S}(0)=298$ & $R_{S}(0)=0$ \\
\hline$S_{M}(0)=500$ & $E_{M}(0)=100$ & $I_{M}(0)=250$ & & \\
\hline
\end{tabular}

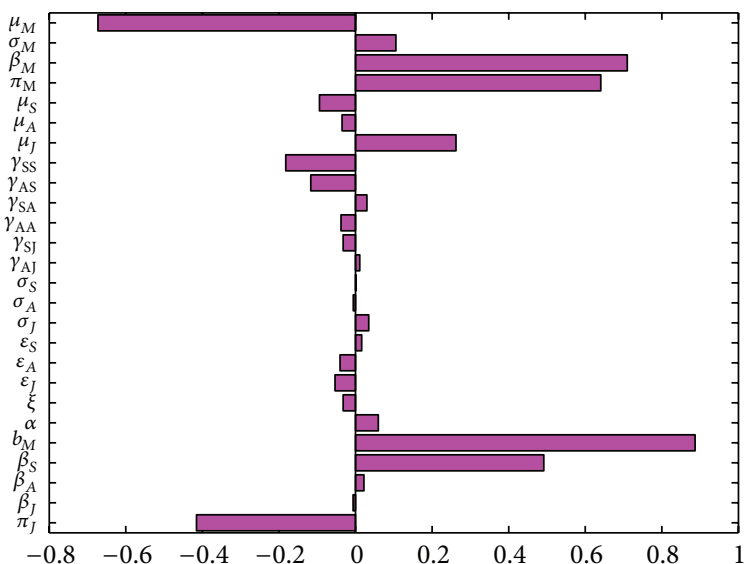

(a)

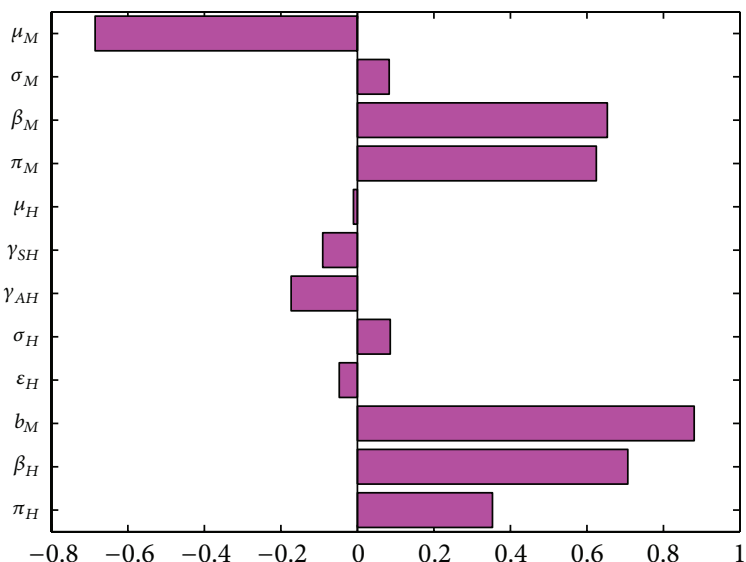

(b)

Figure 6: PRCC values for chikungunya models (10) and (32), using as response functions (a) the reproduction number $\mathscr{R}_{0}$; (b) the reproduction number $\mathscr{R}_{H}$. Parameter values (baseline) and ranges used are as given in Table 3 .

simulations of the model. Figure 6(a) depicts the partial rank correlation coefficient (PRCC) values for each parameter of the models, using the ranges and baseline values tabulated in Table 3 (with the basic reproduction number, $\mathscr{R}_{0}$, as the response function), from which it follows that the parameters that have the most influence on chikungunya transmission dynamics are the mosquito biting rate $\left(b_{M}\right)$, the transmission probability per contact in mosquitoes $\left(\beta_{M}\right)$ and in humans $\left(\beta_{S}\right)$, mosquito recruitment rate $\left(\pi_{M}\right)$, and the death rate of the mosquitoes $\left(\mu_{M}\right)$. It is interesting to note that, from Figure $6(\mathrm{a})$, the transmission probability per contact in juvenile and adult $\left(\beta_{J}\right.$ and $\left.\beta_{A}\right)$ is not as significant as that of the seniors. Thus, this study identifies the most important parameters that drive the transmission mechanism of the disease. The identification of these key parameters is vital to the formulation of effective control strategies for combating the spread of the disease. In other words, the results of this sensitivity analysis suggest that a strategy that reduces the mosquito biting rate (reduces $b_{M}$ ), the mosquito recruitment rate (reduces $\pi_{M}$ ), and the transmission probability per contact in mosquitoes (reduces $\beta_{M}$ ) and in humans (reduces $\beta_{S}$ ) and increases the death rate of the mosquito (increases $\mu_{M}$ ) will be effective in curtailing the spread of chikungunya virus in the community.

The sensitivity analysis was also carried out using model (32) without age-structured with $\mathscr{R}_{H}$, as the response function. The dominant parameters in this case are $b_{M}, \beta_{M}, \beta_{H}$, $\pi_{M}$, and $\mu_{M}$. These results show that the same parameters are dominant for the two response functions $\left(\mathscr{R}_{0}\right.$ and $\left.\mathscr{R}_{H}\right)$. This result further emphasizes the fact that the inclusion of age structure does not alter the dynamics of the transmission of the infection.

\section{Assessment of Control Strategies}

In order to reduce the number of infected human cases, values of some dominant parameters (the mosquito recruitment rate $\left(\pi_{M}\right)$, the death rate of the mosquito $\left(\mu_{M}\right)$, and the transmission probability per contact in mosquitoes $\left(\beta_{M}\right)$ and in humans $\left(\beta_{J}, \beta_{A}\right.$, and $\left.\left.\beta_{S}\right)\right)$ obtained from the sensitivity analysis were adjusted to capture mosquito-reduction strategy, personal-protection strategy, and the effect of the combination of both strategies (universal strategy). The reduction in the mosquito biting rate, a dominant parameter, is captured implicitly by the reduction of all the transmission probabilities. Three effectiveness levels (low, moderate, and high) were evaluated for each of the strategies using the initial conditions in Table 4 obtained from [19]. The numbers in Table 4 were estimated using the 1913 infected individuals obtained in [19], so that $11 \%$ were in the juvenile age group, $73.4 \%$ in the adult group, and $15.6 \%$ in the seniors group age. The susceptible population were a total of 3623 individuals [19]; this population was distributed into juvenile, adult, and senior age groups based on the 2015 India's national age distribution [63], adjusted to match the age profile in [19], so that $31.2 \%$ were juveniles, $60.6 \%$ adults, and $8.3 \%$ seniors. The asymptomatic populations were estimated so that $40 \%$ of the infected juveniles were asymptomatic [16] and $25 \%$ of the infected adults and seniors were asymptomatic, respectively $[17,18]$. Table 5 shows the initial conditions used in the simulation of model (32) without age structure. 
TABLE 5: Initial conditions used in the simulations of model (32) without age structure.

\begin{tabular}{llll}
\hline$S_{H}(0)=3623$ & $E_{H}(0)=611$ & $I_{A H}(0)=510$ & $I_{S H}(0)=1913$ \\
$S_{M}(0)=500$ & $E_{M}(0)=100$ & $I_{M}(0)=250$. & \\
\hline
\end{tabular}

TABLE 6: Simulation results of the cumulative number of new cases at $t=150$ days for the age-structured chikungunya model (10) using mosquito-reduction strategy and the control profile of model (32) without age structure.

\begin{tabular}{lccc}
\hline Humans & Low effectiveness & Moderate effectiveness & High effectiveness \\
\hline Juveniles & 154.1849 & 78.3505 & 16.6460 \\
Adults & 300.8889 & 153.0260 & 32.3984 \\
Seniors & 41.6331 & 21.2259 & 4.4462 \\
Without age structure & 471.2875 & 184.6136 & 51.9926 \\
\hline
\end{tabular}

The low and high effectiveness levels were set to the upper and lower bound, respectively, of the ranges used in the sensitivity analysis and the moderate effectiveness levels were set to the baseline values. It should be pointed out that the parameters values and initial conditions used in these simulations are only of theoretical sense to illustrate the control strategies proposed in this paper.

5.1. Mosquito-Reduction Strategy. A reduction in the birth rates $\left(\pi_{M}\right)$ and the average lifespan $\left(\mu_{M}\right)$ of mosquitoes signifies the effectiveness of adulticiding (such as the use of DDT and indoor residual spraying). For simulation purposes, the following three effectiveness levels of the mosquito-reduction control strategy are considered:

(1) Low effectiveness of the mosquito-reduction strategy: $\pi_{M}=500 \times 0.32 /$ day, $\mu_{M}=(1 / 21) /$ day.

(2) Moderate effectiveness of the mosquito-reduction strategy: $\pi_{M}=500 \times 0.1675 /$ day, $\mu_{M}=(1 / 14) /$ day.

(3) High effectiveness of the mosquito-reduction strategy: $\pi_{M}=500 \times 0.015 /$ day, $\mu_{M}=(1 / 7) /$ day.

The cumulative number of new cases of infections in juveniles, adults, and seniors (see Figure 7) is simulated for the three effectiveness levels of this strategy. A comparison of the three effectiveness levels in all the three age groups in Table 6 at $t=150$ days (the end of simulation period) shows that the high-effectiveness mosquito-reduction strategy led to a considerable reduction in the number of new cases; this is followed by the moderate-effectiveness level and the loweffectiveness level produces the most number of new cases. Thus, there is a clear decrease in the cumulative number of new cases with increasing effectiveness level.

A look at Table 6 shows the control profile of model (32) without age structure; as expected, the inclusion of age structure does not change the overall total number of individuals following the implementation of each control strategy. However, the exclusion fails to show the age distribution that will be required for an age based cost effective control strategy.

5.2. Personal-Protection Strategy. A reduction in the transmission rates $\left(\beta_{J}, \beta_{A}, \beta_{S}, \beta_{M}\right)$ implies the effectiveness of an individual's protection against mosquito bites, thereby implicitly leading to a reduction in mosquito biting rate $\left(b_{M}\right)$.
This is typically achieved by using suitable insect repellents or similar products. The following three effectiveness levels of personal protection are considered:

(1) Low effectiveness of the personal-protection strategy: $\beta_{J}=\beta_{A}=\beta_{S}=\beta_{M}=0.54 /$ day.

(2) Moderate effectiveness of the personal-protection strategy: $\beta_{J}=\beta_{A}=\beta_{S}=\beta_{M}=0.24 /$ day.

(3) High effectiveness of the personal-protection strategy: $\beta_{J}=\beta_{A}=\beta_{S}=\beta_{M}=0.15 /$ day.

Simulations of the age-structured chikungunya model (10) show a decrease in the cumulative number of new cases with increasing levels of effectiveness (see Figure 8). The high effectiveness personal-protection strategy at $t=150$ days (the end of simulation period) led to a considerable reduction in the number of new cases compared to the moderateeffectiveness level (see Table 7) at the same time period. The low-effectiveness level performed the poorest producing the most number of new cases.

The control profile of model (32) without age structure and the overall total number of individuals following the implementation of each control strategy is depicted in Table 7 and as expected, the inclusion of age structure does not change the total number of individuals obtained after implementing each control strategy. Table 7 equally shows the flaw in excluding age distribution in the transmission model, as the model lacking age structure fails to show the age distribution required for an efficient and cost effective age based control strategy.

5.3. Universal Strategy. Simulations for the universal strategy (where both the mosquito-reduction and personalprotection strategies are implemented at once) are assessed by simulation of the model for the following three effectiveness levels:

(1) Low effectiveness of the universal strategy: $\pi_{M}=$ $500 \times 0.32 /$ day, $\mu_{M}=(1 / 21) /$ day, $\beta_{J}=\beta_{A}=\beta_{M}=$ $0.54 /$ day.

(2) Moderate effectiveness of the universal strategy: $\pi_{M}=$ $500 \times 0.1675 /$ day, $\mu_{M}=(1 / 14) /$ day, $\beta_{J}=\beta_{A}=\beta_{M}=$ $0.24 /$ day. 
TABLE 7: Simulation results of the cumulative number of new cases at $t=150$ days for the age-structured chikungunya model (10) using personal-protection strategy and the control profile of model (32) without age structure.

\begin{tabular}{lccc}
\hline Humans & Low effectiveness & Moderate effectiveness & High effectiveness \\
\hline Juveniles & 154.1849 & 98.2295 & 48.7211 \\
Adults & 300.8889 & 192.2149 & 95.3561 \\
Seniors & 41.6331 & 26.8144 & 13.3071 \\
Without age structure & 471.2875 & 192.5576 & 91.4392 \\
\hline
\end{tabular}

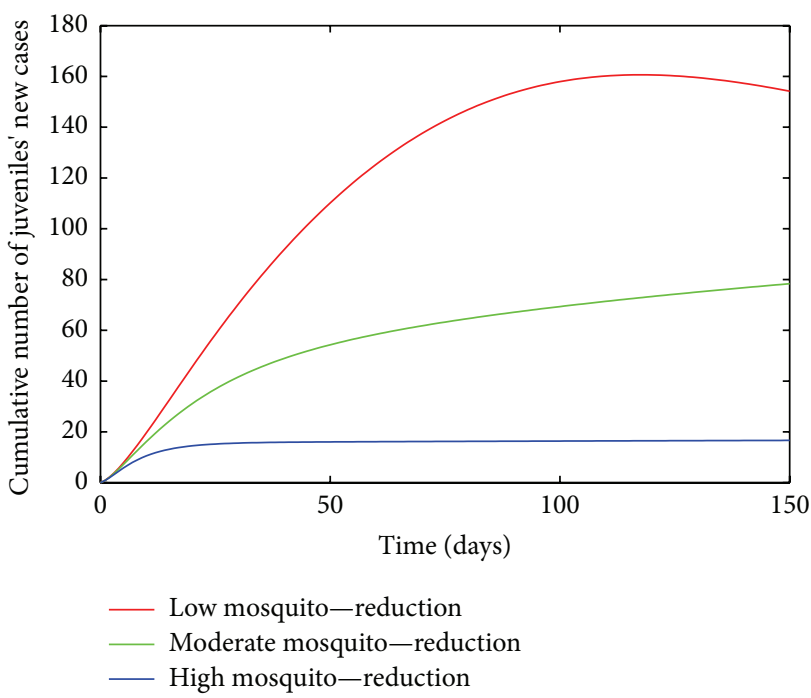

(a)

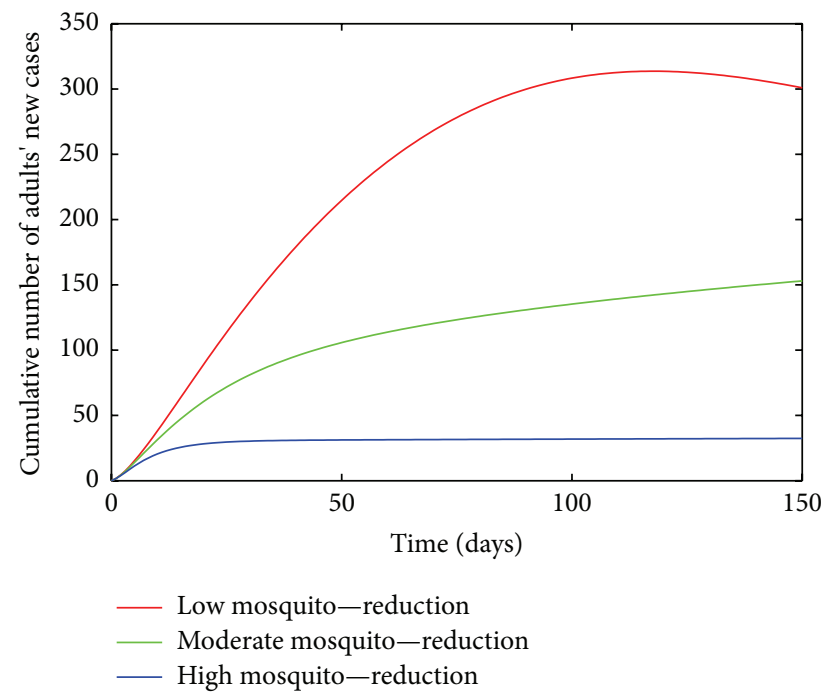

(b)

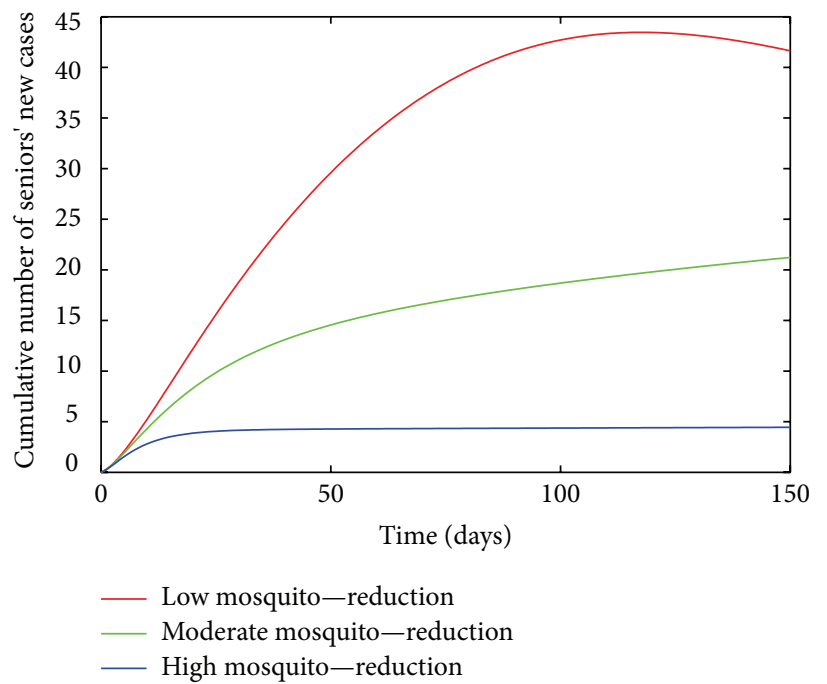

(c)

FIGURE 7: Simulation of age-structured chikungunya model (10) for various effectiveness levels of the mosquito-reduction strategy. (a) The cumulative number of juveniles' new cases. (b) The cumulative number of adults' new cases. (c) The cumulative number of seniors' new cases. Parameter values used are as given in Table 3.

(3) High effectiveness of the universal strategy: $\pi_{M}=$ $500 \times 0.015 /$ day, $\mu_{M}=(1 / 7) /$ day, $\beta_{I}=\beta_{A}=\beta_{M}=$ $0.15 /$ day.

The cumulative number of new cases of infections in juveniles, adults, and seniors is simulated for the three levels of effectiveness for the universal strategy (see Figure 9). A comparison of the three effectiveness levels in Table 8 at $t=150$ days shows that the high-effectiveness level leads to a considerable reduction in the number of new cases; this is followed by the moderate-effectiveness level and the loweffectiveness level produced the most number of new cases. 
TABLE 8: Simulation results of the cumulative number of new cases at $t=150$ days for the age-structured chikungunya model (10) using universal strategy and the control profile of model (32) without age structure.

\begin{tabular}{lccc}
\hline Humans & Low effectiveness & Moderate effectiveness & High effectiveness \\
\hline Juveniles & 154.1849 & 38.0114 & 4.4823 \\
Adults & 300.8889 & 74.2880 & 8.7228 \\
Seniors & 41.6331 & 10.3224 & 1.1966 \\
Without age structure & 471.2875 & 81.0076 & 14.1767 \\
\hline
\end{tabular}

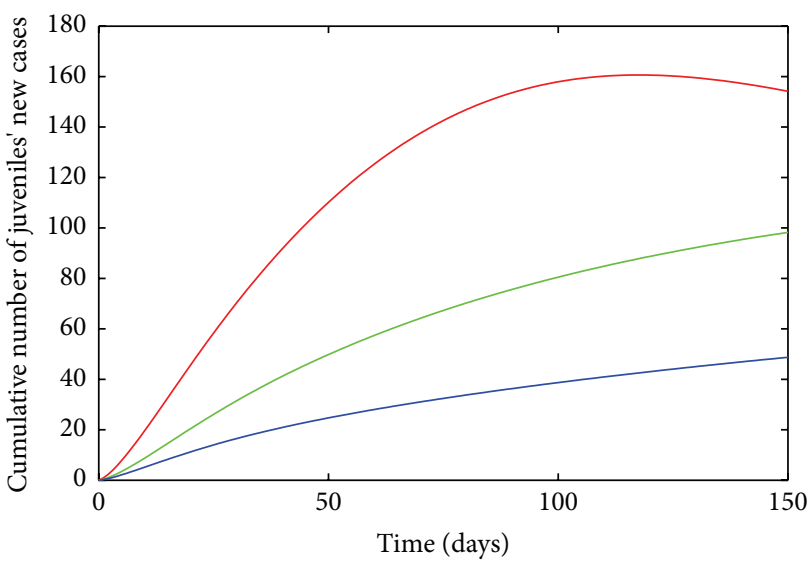

Low personal protection
Moderate personal protection
High personal protection

(a)

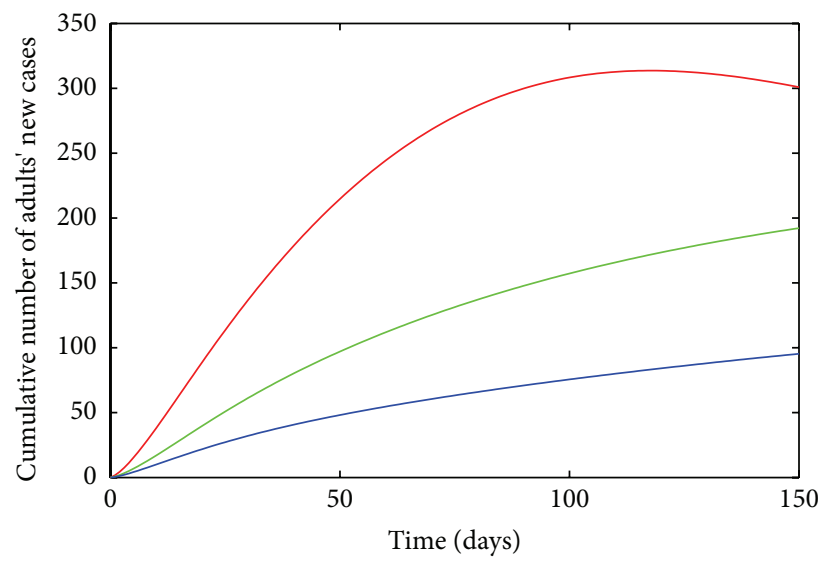

_ Low personal protection

_ Moderate personal protection

_ High personal protection

(b)

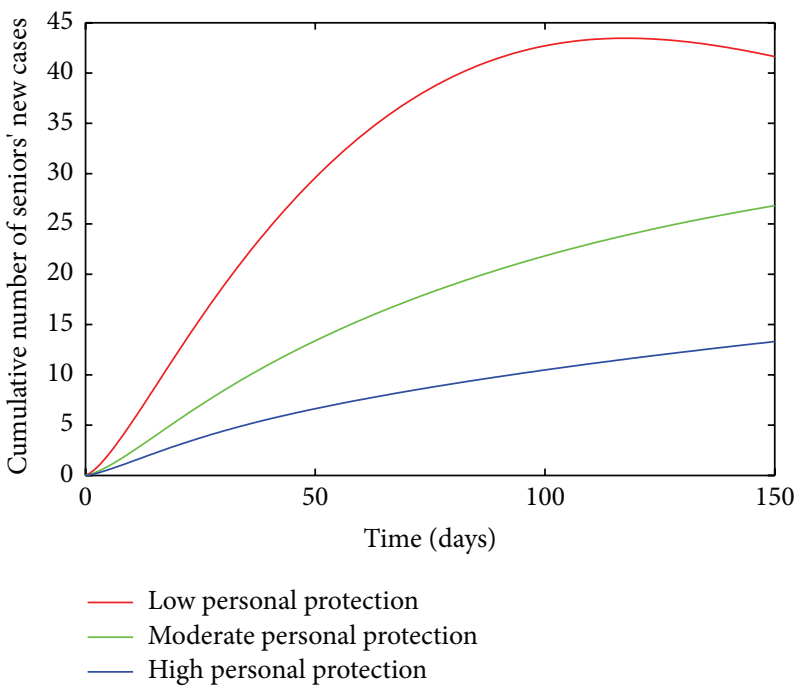

(c)

Figure 8: Simulation of age-structured chikungunya model (10) for various effectiveness levels of the personal-protection strategy. (a) The cumulative number of juveniles' new cases. (b) The cumulative number of adults' new cases. (c) The cumulative number of seniors' new cases. Parameter values used are as given in Table 3.

A comparison of the various high-effectiveness levels of the three control strategies (mosquito-reduction, personalprotection, and universal strategies) in each individual age group at $t=150$ days (see Table 9) shows as expected that the universal strategy is more effective than the other two strategies implemented separately. This is followed by mosquito-reduction strategy which is more effective than the personal protection strategy in reducing chikungunya disease burden.

Table 9 also shows the control profile of model (32) without age structure; as expected, the inclusion of age structure does not change the overall total number of 
TABLE 9: Comparison of the cumulative number of new cases at $t=150$ days for the high-effectiveness levels of the three control strategies for the age-structured chikungunya model (10) and the control profile of model (32) without age structure.

\begin{tabular}{lccc}
\hline $\begin{array}{l}\text { Human } \\
\text { Age groups }\end{array}$ & Mosquito-reduction strategy & Personal-protection strategy & Universal strategy \\
\hline Juveniles & 16.6460 & 48.7211 & 4.4823 \\
Adults & 32.3984 & 95.3561 & 8.7228 \\
Seniors & 4.4462 & 13.3071 & 1.1966 \\
Without age structure & 51.9926 & 91.4392 & 14.1767 \\
\hline
\end{tabular}

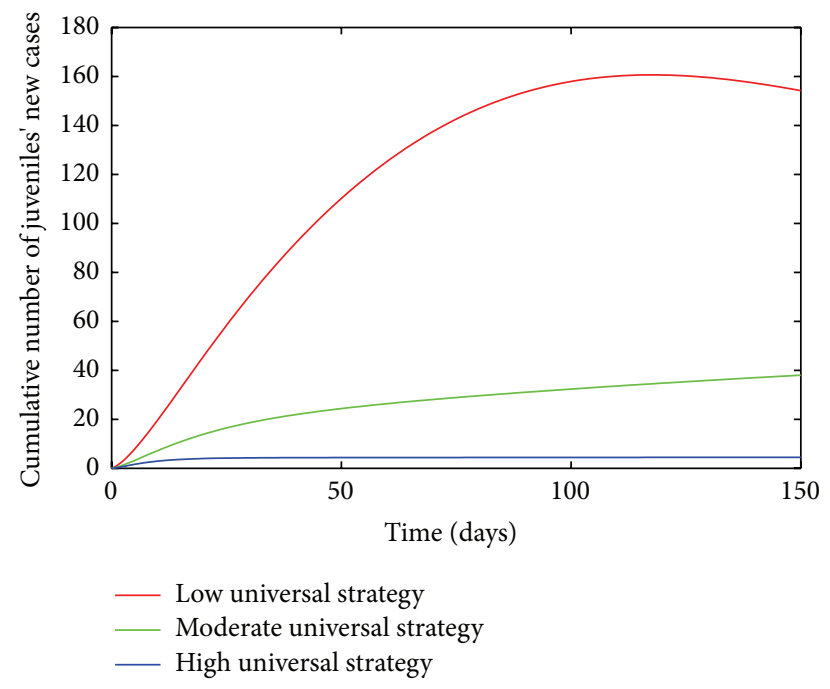

(a)

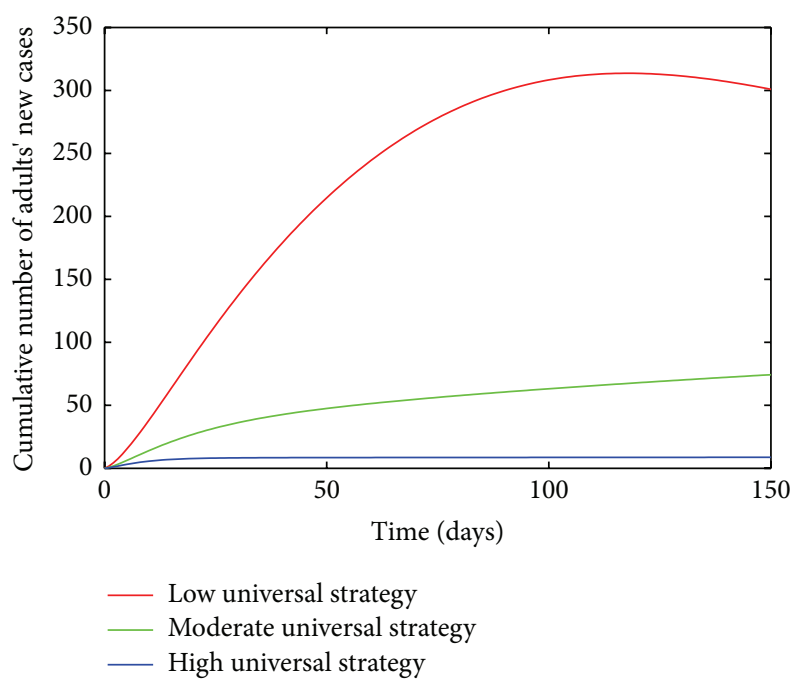

(b)

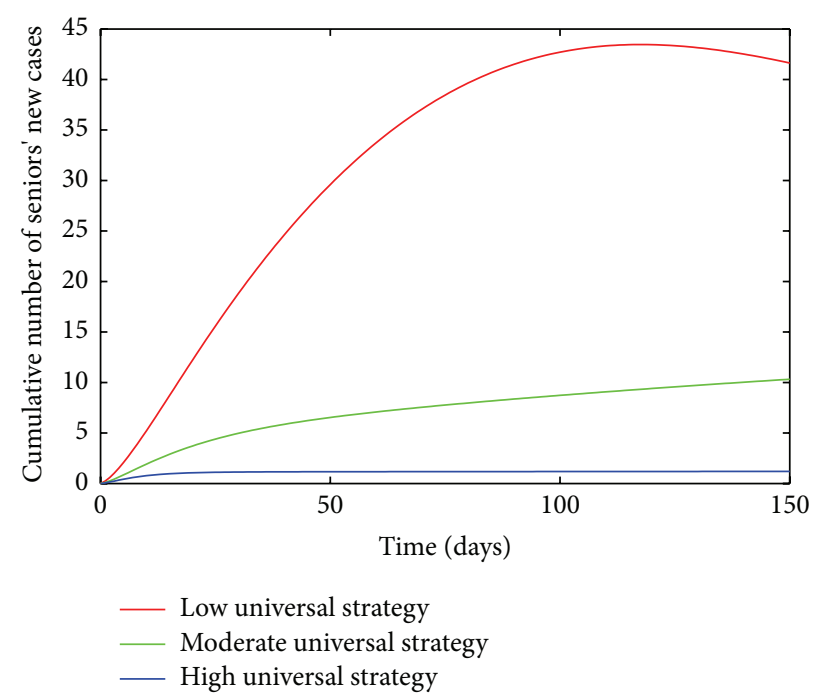

(c)

Figure 9: Simulation of age-structured chikungunya model (10) for various effectiveness levels of the universal strategy. (a) The cumulative number of juveniles' new cases. (b) The cumulative number of adults' new cases. (c) The cumulative number of seniors' new cases. Parameter values used are as given in Table 3.

individuals following the implementation of each control strategy. However, the exclusion fails to show the detailed age distribution that will be required for an effective age based control strategy which in turn will be cost effective.

\section{Discussion and Conclusion}

In this paper, a new deterministic model is designed and used to study the transmission dynamics of an age-structured chikungunya model. The model stratified the population 
by age into juveniles, adults, and seniors and incorporates notable features such as the inclusion of asymptomatic and symptomatic individuals. In order to reduce the number of chikungunya cases, three different control strategies (involving mosquito-reduction strategy, personal-protection strategy, and universal strategy) with three different effectiveness levels (low, moderate, and high) were implemented.

The study shows that the disease-free equilibrium of the model is locally and globally asymptotically stable whenever the associated reproduction number $\left(\mathscr{R}_{0}\right.$, an epidemiological threshold quantity that measures the spreading capacity of the disease) is less than unity and unstable otherwise. The model is shown from this study to exhibit in the presence of disease induced mortality the phenomenon of backward bifurcation, where the stable disease-free equilibrium coexists with a stable endemic equilibrium, when the associated reproduction number is less than unity. Furthermore, the study shows that the inclusion of age structure to the chikungunya virus transmission model does not alter its qualitative dynamics with respect to the local and global stability of the disease-free equilibrium (DFE), as well as with respect to its backward bifurcation property.

This study identifies (via sensitivity analysis) the dominant parameters using as model outcome the basic reproduction number. The parameters with the largest impact are the mosquito biting rate, the transmission probability per contact in mosquitoes and in humans, mosquito recruitment rate, and the death rate of the mosquitoes. The study further shows that the inclusion of age structure does not alter the sensitivity and dominance of the dominant parameters of the chikungunya virus transmission model. The identification of these key parameters is vital to the formulation of effective control strategies for combating the spread of the disease. In other words, the results of this sensitivity analysis suggest that a strategy that reduces the mosquito biting rate, the mosquito recruitment rate, and the transmission probability per contact in mosquitoes and in humans and increases the death rate of the mosquito will be effective in curtailing the spread of chikungunya virus in the community.

Thus, this study shows that even though age distribution is observed in the various epidemics in India $[19,20]$, Thailand [21], and Reunion Islands [5, 22] and across Europe [23], the inclusion of age does not alter the qualitative dynamics of the chikungunya virus transmission model. This implies that the transmission dynamics can be adequately studied without including age structure as observed in the epidemic areas. However, the exclusion of the age structure fails to show the age distribution necessary for adequate and effective control, as shown in Tables 6, 7, 8, and 9. In other words, the model with the exclusion of age structure will lead to a one size fits all blanket control for the entire population.

In order to reduce the number of infected cases, different parameters values were adjusted using the results from the sensitivity analysis. The control strategies were implemented for several cases (mosquito-reduction strategy, personalprotection strategy, and universal strategy) with three different effectiveness levels (low, moderate, and high) using as output measure the cumulative number of new cases of infections in juveniles, adults, and seniors. The results show that the cumulative number of new cases of infections in juveniles, adults, and seniors decreases with increasing effectiveness level, with the high-effectiveness level producing a considerable reduction in the number of new cases. Further comparison of the three control strategies (mosquito reduction, personal-protection, and universal strategies) shows that the universal strategy is more effective in reducing the number of new cases than the other two strategies implemented separately. This is followed by mosquito-reduction strategy which is more effective than the personal-protection strategy in reducing chikungunya disease burden in the community. However, to determine the best and most cost effective strategy, a cost-effectiveness analysis will need to be carried out [64]; this is a future work that is been considered in another paper.

Hence, in this paper, we formulated and analyzed a system of ordinary differential equations for an age-structure transmission dynamics of chikungunya virus. Some of theoretical and epidemiological findings of this study are summarized below:

(i) The age-structured chikungunya model (10) is locally and globally asymptotically stable (LAS) when $\mathscr{R}_{0}<$ 1 and unstable when $\mathscr{R}_{0}>1$.

(ii) The model exhibits in the presence of disease induced mortality the phenomenon of backward bifurcation, where the stable disease-free equilibrium coexists with a stable endemic equilibrium, when the associated reproduction number is less than unity.

(iii) The inclusion of age structure to the chikungunya transmission model (10) does not alter its qualitative dynamics with respect to the local and global stability of the DFE, as well as with respect to its backward bifurcation property.

(iv) The sensitivity analysis of the model shows that the dominant parameters are the mosquito biting rate $\left(b_{M}\right)$, the transmission probability per contact in mosquitoes $\left(\beta_{M}\right)$ and in humans $\left(\beta_{S}\right)$, mosquito recruitment rate $\left(\pi_{M}\right)$, and the death rate of the mosquitoes $\left(\mu_{M}\right)$.

(v) The inclusion of age structure does not alter the sensitivity and dominance of the dominant parameters of the age-structured chikungunya model (10).

(vi) The numerical simulations reveal that the exclusion of age structure fails to show the age distribution needed for an age based effective control strategy, leading to a one size fits all blanket control for the entire population.

(vii) Numerical simulations indicate that mosquito-reduction strategy is more effective than personal-protection strategy, while the universal strategy is the most effective strategy in reducing chikungunya disease burden in the community. 


\section{Appendices}

\section{A. Derivation of (1)}

Humans (susceptible and infected) are bitten by mosquitoes; thus, the average number of mosquito bites humans receive (denoted by $b_{M}$ ) depends on the humans in the community and the total size of the populations of mosquitoes [65]. Thus, it is reasonable to assume that the biting rate $b_{M}$ is constant as female mosquitoes have certain number of blood meals over their lifetime [66]. Let $b_{H}$ be the rate at which bites are received by a single host per unit time. Thus, for the number of bites to be conserved, the following conservation law must hold (i.e., the total number of bites by mosquitoes equals the total number of bites received by humans):

$$
b_{M} N_{M}=b_{H}\left(N_{H}, N_{M}\right) N_{H},
$$

so that

$$
N_{M}=\frac{b_{H}\left(N_{H}, N_{M}\right) N_{H}}{b_{M}} .
$$

Let $\beta_{J} b_{H}$ be the effective contact rate between a susceptible juvenile and infectious mosquitoes, where $\beta_{J}$ is the transmission probability per contact from an infectious mosquito to a susceptible juvenile. Similarly, let $\beta_{M} b_{M}$ be the effective contact rate between a susceptible mosquito and infectious juveniles, where $\beta_{M}$ is the transmission probability per contact from an infectious juvenile to a susceptible mosquito. Thus, susceptible juveniles acquire infection, following effective contact with an infectious vector, at a rate $\lambda_{J}$, given by

$$
\lambda_{J}=\frac{\beta_{J} b_{H}\left(N_{H}, N_{M}\right) I_{M}}{N_{M}} .
$$

Using (A.1) in (A.3) gives

$$
\lambda_{J}=\frac{\beta_{J} b_{M} I_{M}}{N_{H}} .
$$

\section{B. Proof of Lemma 1}

Proof. Let $t_{1}=\sup \{t>0: F(t)>0 \in[0, t]\}$. Thus, $t_{1}>0$. It follows from the first equation of system (10) that

$$
\frac{d S_{J}}{d t}=\pi_{J}-\frac{\beta_{J} b_{M} S_{J} I_{M}}{N_{H}}-\alpha S_{J}-\mu_{J} S_{J},
$$

which can be rewritten as

$$
\begin{gathered}
\frac{d}{d t}\left\{S_{J}(t) \exp \left[\left(\int_{0}^{t_{1}} \frac{\beta_{J} b_{M} I_{M}(\zeta)}{N_{H}(\zeta)} d \zeta+k_{1} t\right)\right]\right\} \\
=\pi_{J} \exp \left[\left(\int_{0}^{t_{1}} \frac{\beta_{J} b_{M} I_{M}(\zeta)}{N_{H}(\zeta)} d \zeta+k_{1} t\right)\right],
\end{gathered}
$$

where $k_{1}=\alpha+\mu_{J}$. Hence,

$$
\begin{gathered}
S_{J}\left(t_{1}\right) \exp \left[\left(\int_{0}^{t_{1}} \frac{\beta_{J} b_{M} I_{M}(\zeta)}{N_{H}(\zeta)} d \zeta+k_{1} t_{1}\right)\right]-S_{J}(0) \\
\quad=\int_{0}^{t_{1}} \pi_{J} \exp \left[\left(\int_{0}^{p} \frac{\beta_{J} b_{M} I_{M}(\zeta)}{N_{H}(\zeta)} d \zeta+k_{1} p\right)\right] d p
\end{gathered}
$$

so that

$$
\begin{aligned}
& S_{J}\left(t_{1}\right)=S_{J}(0) \exp \left[-\left(\int_{0}^{t_{1}} \frac{\beta_{J} b_{M} I_{M}(\zeta)}{N_{H}(\zeta)} d \zeta+k_{1} t_{1}\right)\right] \\
& +\exp \left[-\left(\int_{0}^{t_{1}} \frac{\beta_{J} b_{M} I_{M}(\zeta)}{N_{H}(\zeta)} d \zeta+k_{1} t_{1}\right)\right] \\
& \quad \cdot \int_{0}^{t_{1}} \pi_{J} \exp \left[\left(\int_{0}^{p} \frac{\beta_{J} b_{M} I_{M}(\zeta)}{N_{H}(\zeta)} d \zeta+k_{1} p\right)\right] d p \\
& >0 .
\end{aligned}
$$

Similarly, it can be shown that $F>0$ for all $t>0$.

For the second part of the proof, note that $0<E_{J}(t) \leq$ $N_{H}(t), 0<I_{S J}(t) \leq N_{H}(t), 0<I_{A J}(t) \leq N_{H}(t), 0<R_{J}(t) \leq$ $N_{H}(t), 0<S_{A}(t) \leq N_{H}(t), 0<E_{A}(t) \leq N_{H}(t), 0<I_{A A}(t) \leq$ $N_{H}(t), 0<I_{S A}(t) \leq N_{H}(t), 0<R_{A}(t)<N_{H}(t), 0<S_{S}(t) \leq$ $N_{H}(t), 0<E_{S}(t) \leq N_{H}(t), 0<I_{A S}(t) \leq N_{H}(t), 0<I_{S S}(t) \leq$ $N_{H}(t), 0<R_{S}(t) \leq N_{H}(t), 0<S_{M}(t) \leq N_{M}(t), 0<E_{M}(t) \leq$ $N_{M}(t), 0<I_{M}(t) \leq N_{M}(t)$

Adding the human and mosquito component of the agestructured chikungunya model (10) gives

$$
\begin{aligned}
& \frac{d N_{H}(t)}{d t}=\pi_{J}-\mu_{J} N_{H}(t), \\
& \frac{d N_{M}(t)}{d t}=\pi_{M}-\mu_{M} N_{M}(t),
\end{aligned}
$$

where $\mu_{H}=\min \left\{\mu_{J}, \mu_{A}, \mu_{S}\right\}$.

Hence,

$$
\begin{aligned}
& \frac{\pi_{J}}{\mu_{H}} \leq \liminf _{t \rightarrow \infty} N_{H}(t) \leq \limsup _{t \rightarrow \infty} N_{H}(t)=\frac{\pi_{J}}{\mu_{H}}, \\
& \frac{\pi_{M}}{\mu_{M}} \leq \liminf _{t \rightarrow \infty} N_{M}(t)=\limsup _{t \rightarrow \infty} N_{M}(t)=\frac{\pi_{M}}{\mu_{M}}
\end{aligned}
$$

as required.

\section{Proof of Lemma 2}

Proof. The following steps are followed to establish the positive invariance of $\Omega$ (i.e., solutions in $\Omega$ remain in $\Omega$ for all $t>0$ ). The rate of change of the total population is obtained by adding the human and mosquito component of the agestructured chikungunya model (10) to give

$$
\begin{aligned}
& \frac{d N_{H}(t)}{d t}=\pi_{J}-\mu_{H} N_{H}(t), \\
& \frac{d N_{M}(t)}{d t}=\pi_{M}-\mu_{M} N_{M}(t),
\end{aligned}
$$

where $\mu_{H}=\min \left\{\mu_{J}, \mu_{A}, \mu_{S}\right\}$.

A standard comparison theorem [67] can then be used to show that $N_{H}(t) \leq N_{H}(0) e^{-\mu_{H} t}+\left(\pi_{J} / \mu_{H}\right)\left(1-e^{-\mu_{H} t}\right)$ and $N_{M}(t) \leq N_{M}(0) e^{-\mu_{M} t}+\left(\pi_{M} / \mu_{M}\right)\left(1-e^{-\mu_{M} t}\right)$. In particular, $N_{H}(t) \leq \pi_{J} / \mu_{H}$, if $N_{H}(0) \leq \pi_{J} / \mu_{H}$ and $N_{M}(t) \leq \pi_{M} / \mu_{M}$, if $N_{M}(0) \leq \pi_{M} / \mu_{M}$. Thus, the region $\Omega$ is positively invariant. 
Hence, it is sufficient to consider the dynamics of the flow generated by (10) in $\Omega$. In this region, the model is epidemiologically and mathematically well posed [45]. Thus, every solution of the age-structured chikungunya model (10) with initial conditions in $\Omega$ remains in $\Omega$ for all $t>0$. Therefore, the $\omega$-limit sets of the system (10) are contained in $\Omega$. This result is summarized below.

\section{Proof of Lemma 4}

Proof. It follows from the first equation of age-structured chikungunya model $(10)$ (where $S_{J}^{*}=\pi_{J} /\left(\alpha+\mu_{J}\right)$ ) that

$$
\begin{aligned}
\frac{d S_{J}(t)}{d t} & =\pi_{J}-\frac{\beta_{J} b_{M} S_{J} I_{M}}{N_{H}}-\left(\alpha+\mu_{J}\right) S_{J}(t) \\
& \leq \pi_{J}-\left(\alpha+\mu_{J}\right) S_{J}(t) \\
& \leq\left(\alpha+\mu_{J}\right)\left[\frac{\pi_{J}}{\left(\alpha+\mu_{H}\right)}-S_{J}(t)\right] \\
& =\left(\alpha+\mu_{J}\right)\left[S_{J}^{*}-S_{J}(t)\right] .
\end{aligned}
$$

Hence,

$$
S_{J}(t) \leq S_{J}^{*}-\left[S_{J}^{*}-S_{J}(0)\right] e^{-\left(\alpha+\mu_{J}\right) t}
$$

Thus, if $N_{H}^{*}=\Pi_{J} / \mu_{H}$ and $S_{J}(0) \leq S_{J}^{*}$ for all $t \geq 0$, then $S_{J}(t) \leq S_{J}^{*}$ for all $t \geq 0$.

Similarly, it follows from the sixth equation of agestructured chikungunya model (10) (where $S_{A}^{*}=\left(\pi_{J} /(\alpha+\right.$ $\left.\left.\mu_{J}\right)\right)\left(\alpha /\left(\xi+\mu_{A}\right)\right)$ that

$$
\begin{aligned}
\frac{d S_{A}(t)}{d t} & =\alpha S_{J}(t)-\frac{\beta_{A} b_{M} S_{A} I_{M}}{N_{H}}-\left(\xi+\mu_{A}\right) S_{A}(t) \\
& \leq \alpha S_{J}(t)-\left(\xi+\mu_{A}\right) S_{A}(t) \\
& \leq \alpha S_{J}^{*}-\left(\xi+\mu_{A}\right) S_{A}(t), \quad \text { since } S_{J}<S_{J}^{*} \\
& \leq \alpha \frac{\pi_{J}}{\left(\alpha+\mu_{J}\right)}-\left(\xi+\mu_{A}\right) S_{A}(t) \\
& \leq\left(\xi+\mu_{H}\right)\left[\frac{\pi_{J}}{\left(\alpha+\mu_{J}\right)} \frac{\alpha}{\left(\xi+\mu_{A}\right)}-S_{A}(t)\right] \\
& =\left(\xi+\mu_{A}\right)\left[S_{A}^{*}-S_{A}(t)\right] .
\end{aligned}
$$

Thus,

$$
S_{A}(t) \leq S_{A}^{*}-\left[S_{A}^{*}-S_{A}(0)\right] e^{-\left(\xi+\mu_{A}\right) t} .
$$

Thus, if $S_{A}(0) \leq S_{A}^{*}$ for all $t \geq 0$, then $S_{A}(t) \leq S_{A}^{*}$ for all $t \geq 0$.
Furthermore, it follows from the eleventh equation of agestructured chikungunya model (10) (where $S_{S}^{*}=\xi \pi_{J} \alpha / \mu_{S}(\alpha+$ $\left.\left.\mu_{J}\right)\left(\xi+\mu_{A}\right)\right)$ that

$$
\begin{aligned}
\frac{d S_{S}(t)}{d t} & =\xi S_{A}(t)-\frac{\beta_{S} b_{M} S_{S} I_{M}}{N_{H}}-\mu_{S} S_{S}(t) \\
& \leq \xi S_{A}(t)-\mu_{S} S_{S}(t) \leq \xi S_{A}^{*}(t)-\mu_{S} S_{S}(t) \\
& \text { since } S_{A}< \\
& \leq \xi \frac{\pi_{J} \alpha}{\left(\alpha+\mu_{J}\right)\left(\xi+\mu_{A}\right)}-\mu_{S} S_{S}(t) \\
& \leq \mu_{S}\left[\frac{\xi \pi_{J} \alpha}{\mu_{S}\left(\alpha+\mu_{J}\right)\left(\xi+\mu_{A}\right)}-S_{S}(t)\right] \\
& =\mu_{S}\left[S_{S}^{*}-S_{S}(t)\right] .
\end{aligned}
$$$$
\text { since } S_{A}<S_{A}^{*}
$$

Thus,

$$
S_{S}(t) \leq S_{S}^{*}-\left[S_{S}^{*}-S_{S}(0)\right] e^{-\mu_{S} t} .
$$

Hence, if $S_{S}(0) \leq S_{S}^{*}$ for all $t \geq 0$, then $S_{S}(t) \leq S_{S}^{*}$ for all $t \geq 0$.

Finally, it follows from the fifteenth equation of agestructured chikungunya model (10) that

$$
\begin{aligned}
& \frac{d S_{M}(t)}{d t} \\
& =\pi_{M} \\
& \quad-\beta_{M} b_{M}\left[\frac{I_{A J}+I_{S J}+I_{A A}+I_{S A}+I_{A S}+I_{S S}}{N_{H}}\right] S_{M} \\
& \quad-\mu_{M} S_{M}(t) \leq \pi_{M}-\mu_{M} S_{M}(t) \\
& \leq \mu_{M}\left[\frac{\pi_{M}}{\mu_{M}}-S_{M}(t)\right]=\mu_{M}\left[S_{M}^{*}-S_{M}(t)\right] .
\end{aligned}
$$

Thus,

$$
S_{M}(t) \leq S_{M}^{*}-\left[S_{M}^{*}-S_{M}(0)\right] e^{-\mu_{M} t} .
$$

Hence, if $N_{M}^{*}=\pi_{M} / \mu_{M}$ and $S_{M}(0) \leq S_{M}^{*}$ for all $t \geq 0$, then $S_{M}(t) \leq S_{M}^{*}$ for all $t \geq 0$.

Thus, in summary, it has been shown that the region $\Omega_{1}$ is positively invariant and attracts all solutions in $\mathbb{R}_{+}^{18}$ for the age-structured model (10).

\section{E. Proof of Theorem 5}

Proof. To prove the global stability of the DFE, we will follow the approach in [68].

Let $X=\left(S_{J}, R_{J}, S_{A}, R_{A}, S_{S}, R_{S}, S_{M}\right)$ and $Z=\left(E_{J}, I_{A J}, I_{S J}\right.$, $\left.E_{A}, I_{A A}, I_{S A}, E_{S}, I_{A S}, I_{S S}, E_{M}, I_{M}\right)$ and group system (10) into

$$
\begin{aligned}
& \frac{d X}{d t}=F(X, 0), \\
& \frac{d Z}{d t}=G(X, Z),
\end{aligned}
$$


where $F(X, 0)$ is the right hand side of $\dot{S}_{J}, \dot{R}_{J}, \dot{S_{A}}, \dot{R_{A}}, \dot{S_{S}}, \dot{R_{S}}$, and $S_{M}$ with $E_{J}=I_{A J}=I_{S J}=E_{A}=I_{A A}=I_{S A}=E_{S}=I_{A S}=$ $I_{S S}=E_{M}=I_{M}=0$ and $G(X, Z)$ is the right hand side of $\dot{E}_{J}$, $I_{A J}^{\dot{I}}, \dot{I_{S S}}, \dot{E_{A}}, I_{A A}, \dot{I_{S A}}, \dot{E_{S}}, I_{A S}, I_{S S}, \dot{E_{M}}$, and $I_{M}$.

Next, consider the reduced system $d X / d t=F(X, 0)$ given as follows:

$$
\begin{aligned}
\frac{d S_{J}}{d t} & =\pi_{J}-\left(\alpha+\mu_{J}\right) S_{J}, \\
\frac{d R_{J}}{d t} & =-\left(\alpha+\mu_{J}\right) R_{J}, \\
\frac{d S_{A}}{d t} & =\alpha S_{J}-\left(\xi+\mu_{A}\right) S_{A}, \\
\frac{d R_{A}}{d t} & =\alpha R_{J}-\left(\xi+\mu_{A}\right) R_{A}, \\
\frac{d S_{S}}{d t} & =\xi S_{A}-\mu_{S} S_{S}, \\
\frac{d R_{S}}{d t} & =\xi R_{A}-\mu_{S} R_{S}, \\
\frac{d S_{M}}{d t} & =\pi_{M}-\mu_{M} S_{M} .
\end{aligned}
$$

Let

$$
\begin{aligned}
X^{*} & =\left(S_{J}^{*}, R_{J}^{*}, S_{A}^{*}, R_{A}^{*}, S_{S}^{*}, R_{S}^{*}, S_{M}^{*}\right) \\
& =\left(\frac{\pi_{J}}{\alpha+\mu_{J}}, 0, \frac{\alpha S_{J}^{*}}{\left(\xi+\mu_{A}\right)}, 0, \frac{\xi S_{A}^{*}}{\mu_{S}}, 0, \frac{\pi_{M}}{\mu_{M}}\right)
\end{aligned}
$$

be an equilibrium of the reduced system (E.2) and (E.3); we show that $X^{*}$ is a globally stable equilibrium in $\Omega_{1}$.

To do this, solve the first and second equation of (E.2) and (E.3); this gives $S_{J}(t)=\pi_{J} / l_{1}+e^{-l_{1} t}\left[S_{J}(0)-\pi_{J} / l_{1}\right]$, $R_{J}(t)=R_{J}(0) e^{-l_{1} t}$ (where $l_{1}=\alpha+\mu_{J}$ ), which approaches $\pi_{J} / l_{1}$ and zero, respectively, as $t \rightarrow \infty$. Next, solving for $S_{A}(t)$ and $R_{A}(t)$ and using $S_{J}(t)$ and $R_{J}(t)$ in (E.2) and (E.3) give $S_{A}(t)=\left[\int_{0}^{t} \alpha S_{J}(z) e^{l_{2} z} d z+S_{A}(0)\right] e^{-l_{2} t}$ and $R_{A}(t)=$ $\left[\int_{0}^{t} \alpha R_{J}(z) e^{l_{2} z} d z+R_{A}(0)\right] e^{-l_{2} t}$ (where $l_{2}=\xi+\mu_{A}$ ), which converges, respectively, to $\pi_{J} \alpha / l_{1} l_{2}$ and zero as $t \rightarrow \infty$. Similarly, solving for $S_{S}(t)$ and $R_{S}(t)$ and using $S_{A}(t)$ and $R_{A}(t)$ in (E.2) give $S_{S}(t)=\left[\int_{0}^{t} \xi S_{A}(z) e^{\mu_{S} z} d z+S_{S}(0)\right] e^{-\mu_{S} t}$ and $R_{S}(t)=\left[\int_{0}^{t} \alpha R_{A}(z) e^{\mu_{S} z} d z+R_{S}(0)\right] e^{-\mu_{S} t}$ which converges to $\pi_{J} \alpha \xi / l_{1} l_{2} \mu_{S}$ and zero as $t \rightarrow \infty$. Lastly solving for $S_{M}(t)$ in (E.2) gives $S_{M}(t)=\pi_{M} / \mu_{M}+e^{-\mu_{M} t}\left[S_{M}(0)-\pi_{M} / \mu_{M}\right]$ which converges to $\pi_{M} / \mu_{M}$, as $t \rightarrow \infty$.

These asymptotic dynamics are independent of initial conditions in $\Omega$. Hence, the convergence of solutions of (E.2) and (E.3) is global in $\Omega_{1}$. Next, we require $G(X, Z)$ to satisfy the following two conditions given in [69, page 246], namely,

(i) $G(X, 0)=0$;

(ii) $G(X, Z)=D_{Z} G\left(X^{*}, 0\right) Z-\widehat{G}(X, Z), \widehat{G}(X, Z) \geq 0$,

where $\left(X^{*}, 0\right)=\left(\pi_{J} /\left(\alpha+\mu_{J}\right), 0, \alpha S_{J}^{*} /\left(\xi+\mu_{A}\right), 0, \xi S_{A}^{*} / \mu_{S}, 0\right.$, $\left.\pi_{M} / \mu_{M}, 0,0,0,0,0,0,0,0,0,0,0\right)$ and $D_{Z} G\left(X^{*}, 0\right)$ is the Jacobian of $G(X, Z)$ taken with respect to $\left(E_{J}, I_{A J}, I_{S J}, E_{A}\right.$, $\left.I_{A A}, I_{S A}, E_{S}, I_{A S}, I_{S S}, E_{M}, I_{M}\right)$ and evaluated at $\left(X^{*}, 0\right)$, which is an $M$-matrix (the off diagonal elements are nonnegative). Thus,

$$
D_{Z} G\left(X^{*}, 0\right)=\left(\begin{array}{ccccccccccc}
-k_{2} & 0 & 0 & 0 & 0 & 0 & 0 & 0 & 0 & 0 & \Psi_{J} \\
\varepsilon_{J} \sigma_{J} & -k_{3} & 0 & 0 & 0 & 0 & 0 & 0 & 0 & 0 & 0 \\
\left(1-\varepsilon_{J}\right) \sigma_{J} & 0 & -k_{4} & 0 & 0 & 0 & 0 & 0 & 0 & 0 & 0 \\
\alpha & 0 & 0 & -k_{7} & 0 & 0 & 0 & 0 & 0 & 0 & \Psi_{A} \\
0 & \alpha & 0 & \varepsilon_{A} \sigma_{A} & -k_{8} & 0 & 0 & 0 & 0 & 0 & 0 \\
0 & 0 & \alpha & \left(1-\varepsilon_{A}\right) \sigma_{A} & 0 & -k_{9} & 0 & 0 & 0 & 0 & 0 \\
0 & 0 & 0 & \xi & 0 & 0 & -k_{11} & 0 & 0 & 0 & \Psi_{S} \\
0 & 0 & 0 & 0 & \xi & 0 & \varepsilon_{S} \sigma_{S} & -k_{12} & 0 & 0 & 0 \\
0 & 0 & 0 & 0 & 0 & \xi & \left(1-\varepsilon_{S}\right) \sigma_{S} & 0 & -k_{13} & 0 & 0 \\
0 & \Psi_{M} & \Psi_{M} & 0 & \Psi_{M} & \Psi_{M} & 0 & \Psi_{M} & \Psi_{M} & -k_{14} & 0 \\
0 & 0 & 0 & 0 & 0 & 0 & 0 & 0 & 0 & \sigma_{M} & -\mu_{M}
\end{array}\right),
$$


where $\Psi_{J}=\beta_{J} b_{M} S_{J}^{*} / N_{H}^{*}, \Psi_{A}=\beta_{A} b_{M} S_{A}^{*} / N_{H}^{*}, \Psi_{S}=\beta_{S} b_{M} S_{S}^{*} /$ $N_{H}^{*}, \Psi_{M}=b_{M} \beta_{M} S_{M}^{*} / N_{H}^{*}$, and

$$
\widehat{G}(X, Z)=\left(\begin{array}{ccccccccccc}
0 & 0 & 0 & 0 & 0 & 0 & 0 & 0 & 0 & 0 & \Phi_{J} I_{M} \\
0 & 0 & 0 & 0 & 0 & 0 & 0 & 0 & 0 & 0 & 0 \\
0 & 0 & 0 & 0 & 0 & 0 & 0 & 0 & 0 & 0 & 0 \\
0 & 0 & 0 & 0 & 0 & 0 & 0 & 0 & 0 & 0 & \Phi_{A} I_{M} \\
0 & 0 & 0 & 0 & 0 & 0 & 0 & 0 & 0 & 0 & 0 \\
0 & 0 & 0 & 0 & 0 & 0 & 0 & 0 & 0 & 0 & 0 \\
0 & 0 & 0 & 0 & 0 & 0 & 0 & 0 & 0 & 0 & \Phi_{S} I_{M} \\
0 & 0 & 0 & 0 & 0 & 0 & 0 & 0 & 0 & 0 & 0 \\
0 & 0 & 0 & 0 & 0 & 0 & 0 & 0 & 0 & 0 & 0 \\
0 & \Phi_{M} I_{A J} & \Phi_{M} I_{S J} & 0 & \Phi_{M} I_{A A} & \Phi_{M} I_{S A} & 0 & \Phi_{M} I_{A S} & \Phi_{M} I_{S S} & 0 & 0 \\
0 & 0 & 0 & 0 & 0 & 0 & 0 & 0 & 0 & 0 & 0
\end{array}\right),
$$

where $\Phi_{J}=\beta_{J} b_{M}\left(S_{J}^{*} / N_{H}^{*}\right)\left(\left(1-\left(N_{H}^{*} / S_{J}^{*}\right)\left(S_{J} / N_{H}\right)\right), \Phi_{A}=\right.$ $\beta_{A} b_{M}\left(S_{A}^{*} / N_{H}^{*}\right)\left(1-\left(N_{H}^{*} / S_{A}^{*}\right)\left(S_{A} / N_{H}\right)\right), \Phi_{S}=\beta_{S} b_{M}\left(S_{S}^{*} /\right.$ $\left.N_{H}^{*}\right)\left(1-\left(N_{H}^{*} / S_{S}^{*}\right)\left(S_{S} / N_{H}\right)\right)$, and $\Phi_{M}=b_{M} \beta_{M}\left(S_{M}^{*} / N_{H}^{*}\right)(1-$ $\left.\left(N_{H}^{*} S_{M} / S_{M}^{*} N_{H}\right)\right)$. Furthermore, $S_{J}^{*}=\left(\pi_{J} / k_{1}\right), S_{A}^{*}=\left(\pi_{J} \alpha /\right.$ $\left.k_{1} k_{6}\right), S_{S}^{*}=\left(\pi_{J} \alpha \xi / k_{1} k_{6} \mu_{H}\right), N_{H}^{*}=\left(\pi_{H} / \mu_{H}\right)$, and $S_{M}^{*}=$ $\left(\pi_{M} / \mu_{M}\right)$. We have in $\Omega_{1}$ that, $S_{J} \leq S_{J}^{*}, S_{A} \leq S_{A}^{*}, S_{S} \leq S_{S}^{*}$, and also $S_{M} \leq S_{M}^{*}$. Thus, if the human population is at equilibrium level, it follows that $\left(1-\left(N_{H}^{*} / S_{J}^{*}\right)\left(S_{J} / N_{H}\right)\right)>0$, $\left(1-\left(N_{H}^{*} / S_{A}^{*}\right)\left(S_{A} / N_{H}\right)\right)>0,\left(1-\left(N_{H}^{*} / S_{S}^{*}\right)\left(S_{S} / N_{H}\right)\right)>0$, and
$\left(1-\left(S_{M} N_{H}^{*} / N_{H} S_{M}^{*}\right)\right)>0$; hence $\widehat{G}_{2}(X, Z) \geq 0$. Therefore, by the theorem in [69, page 246], the disease-free equilibrium is globally asymptotically stable since in the absence of disease induced mortality the human population is constant.

\section{F. Components of the Equilibrium $\mathscr{E}_{1}$ of Model (29)}

Consider

$$
\begin{aligned}
S_{J}^{* *} & =\frac{\pi_{J}^{2}}{\left(\beta_{J} b_{M} I_{M}^{* *} \mu_{H}+k_{1} \pi_{J}\right)}, \\
E_{J}^{* *} & =\frac{\beta_{J} b_{M} I_{M}^{* *} \mu_{H} \pi_{J}}{k_{2}\left(\beta_{J} b_{M} I_{M}^{* *} \mu_{H}+k_{1} \pi_{J}\right)}, \\
I_{A J}^{* *} & =\frac{\varepsilon_{J} \sigma_{J} \beta_{J} b_{M} I_{M}^{* *} \mu_{H} \pi_{J}}{k_{3} k_{2}\left(\beta_{J} b_{M} I_{M}^{* *} \mu_{H}+k_{1} \pi_{J}\right)}, \\
I_{S J}^{* *} & =\frac{\left(1-\varepsilon_{J}\right) \sigma_{J} \beta_{J} b_{M} I_{M}^{* *} \mu_{H} \pi_{J}}{k_{4} k_{2}\left(\beta_{J} b_{M} I_{M}^{* *} \mu_{H}+k_{1} \pi_{J}\right)}, \\
R_{J}^{* *} & =\frac{\sigma_{J} \beta_{J} b_{M} I_{M}^{* *} \mu_{H} \pi_{J}\left[\gamma_{A J} k_{4} \varepsilon_{J}+\gamma_{S J}\left(1-\varepsilon_{J}\right) k_{3}\right]}{k_{5} k_{4} k_{3} k_{2}\left(\beta_{J} b_{M} I_{M}^{* *} \mu_{H}+k_{1} \pi_{J}\right)}, \\
S_{A}^{* *}= & \frac{\alpha S_{J}^{* *} \pi_{J}}{\left(\beta_{A} b_{M} I_{M}^{* * *} \mu_{H}+\pi_{J} k_{6}\right)}, \\
E_{A}^{* *}= & \frac{\alpha\left(E_{J}^{* *} \beta_{A} b_{M} I_{M}^{* *} \mu_{H}+E_{J}^{* *} \pi_{J} k_{6}+\beta_{A} b_{M} I_{M}^{* *} \mu_{H} S_{J}^{* *}\right)}{k_{7}\left(\beta_{A} b_{M} I_{M}^{* *} \mu_{H}+\pi_{J} k_{6}\right)}, \\
I_{A A}^{* *}= & \frac{\alpha\left[I_{A J}^{* *} k_{7} \beta_{A} b_{M} I_{M}^{* *} \mu_{H}+I_{A J}^{* *} k_{7} \pi_{J} k_{6}+\varepsilon_{A} \sigma_{A} E_{J}^{* *} \beta_{A} b_{M} I_{M}^{* *} \mu_{H}+\varepsilon_{A} \sigma_{A} E_{J}^{* *} \pi_{J} k_{6}+\varepsilon_{A} \sigma_{A} \beta_{A} b_{M} I_{M}^{* *} \mu_{H} S_{J}^{* *}\right]}{\left[k_{8} k_{7}\left(\beta_{A} b_{M} I_{M}^{* *} \mu_{H}+\pi_{J} k_{6}\right)\right]},
\end{aligned}
$$




$$
\begin{aligned}
& I_{S A}^{* *} \\
& =\frac{\alpha\left[I_{S J}^{* *} k_{7} \beta_{A} b_{M} I_{M}^{* *} \mu_{H}+I_{S J}^{* *} k_{7} \pi_{J} k_{6}+\left(1-\varepsilon_{J}\right) \sigma_{A} E_{J}^{* *} \beta_{A} b_{M} I_{M}^{* *} \mu_{H}+\left(1-\varepsilon_{J}\right) \sigma_{A} E_{J}^{* *} \pi_{J} k_{6}+\left(1-\varepsilon_{J}\right) \sigma_{A} \beta_{A} b_{M} I_{M}^{* *} \mu_{H} S_{J}^{* *}\right]}{\left[k_{9} k_{7}\left(\beta_{A} b_{M} I_{M}^{* *} \mu_{H}+\pi_{J} k_{6}\right)\right]}, \\
& R_{A}^{* *}=\alpha\left[\gamma_{A A} k_{9} I_{A J}^{* *} k_{7} \beta_{A} b_{M} I_{M}^{* *} \mu_{H}+\gamma_{A A} k_{9} I_{A J}^{* *} k_{7} \pi_{J} k_{6}+\gamma_{A A} k_{9} \varepsilon_{A} \sigma_{A} E_{J}^{* *} \beta_{A} b_{M} I_{M}^{* * *} \mu_{H}+\gamma_{A A} k_{9} \varepsilon_{A} \sigma_{A} E_{J}^{* *} \pi_{J} k_{6}\right. \\
& +\gamma_{A A} k_{9} \varepsilon_{A} \sigma_{A} \beta_{A} b_{M} I_{M}^{* *} \mu_{H} S_{J}^{* *}+\gamma_{S A} I_{S J}^{* *} k_{7} \beta_{A} b_{M} I_{M}^{* *} \mu_{H} k_{8}+\gamma_{S A} I_{S J}^{* *} k_{7} \pi_{J} k_{6} k_{8}+\gamma_{S A}\left(1-\varepsilon_{J}\right) \sigma_{A} E_{J}^{* *} \beta_{A} b_{M} I_{M}^{* *} \mu_{H} k_{8} \\
& \left.+\gamma_{S A}\left(1-\varepsilon_{J}\right) \sigma_{A} E_{J}^{* *} \pi_{J} k_{6} k_{8}+\gamma_{S A}\left(1-\varepsilon_{J}\right) \sigma_{A} \beta_{A} b_{M} I_{M}^{* *} \mu_{H} S_{J}^{* *} k_{8}+R_{J}^{* *} k_{9} k_{7} \beta_{A} b_{M} I_{M}^{* *} \mu_{H} k_{8}+R_{J}^{* *} k_{9} k_{7} \pi_{J} k_{6} k_{8}\right] \\
& \cdot\left[k_{10} k_{9} k_{7} k_{8}\left(\beta_{A} b_{M} I_{M}^{* *} \mu_{H}+\pi_{J} k_{6}\right)\right]^{-1} \\
& S_{S}^{* *}=\frac{\xi \pi_{J} S_{A}^{* *}}{\left(\beta_{S} b_{M} I_{M}^{* *} \mu_{H}+\pi_{J} \mu_{S}\right)}, \\
& E_{S}^{* *}=\frac{\xi\left[\beta_{S} b_{M} I_{M}^{* *} \mu_{H} S_{A}^{* *}+\left(\beta_{S} b_{M} I_{M}^{* *} \mu_{H}+\pi_{J} \mu_{S}\right) E_{A}^{* *}\right]}{k_{11}\left(\beta_{S} b_{M} I_{M}^{* *} \mu_{H}+\pi_{J} \mu_{S}\right)}, \\
& I_{A S}^{* *}=\frac{\xi\left[\varepsilon_{S} \sigma_{S} \beta_{S} b_{M} I_{M}^{* *} \mu_{S} S_{A}^{* *}+\left(\beta_{S} b_{M} I_{M}^{* *} \mu_{H}+\pi_{J} \mu_{S}\right)\left(\varepsilon_{S} \sigma_{S} E_{A}^{* *}+I_{A A}^{* *} k_{11}\right)\right]}{k_{12} k_{11}\left(\beta_{S} b_{M} I_{M}^{* * *} \mu_{H}+\pi_{J} \mu_{S}\right)}, \\
& I_{S S}^{* *}=\frac{\xi\left\{\left(1-\varepsilon_{S}\right) \sigma_{S} \beta_{S} b_{M} I_{M}^{* *} \mu_{H} S_{A}^{* *}+\left(\beta_{S} b_{M} I_{M}^{* *} \mu_{H}+\pi_{J} \mu_{S}\right)\left[\left(1-\varepsilon_{S}\right) \sigma_{S} E_{A}^{* *}+I_{S A}^{* *} k_{11}\right]\right\}}{\left[k_{13} k_{11}\left(\beta_{S} b_{M} I_{M}^{* *} \mu_{H}+\pi_{J} \mu_{S}\right)\right]}, \\
& R_{S}^{* *}=\xi\left\{\gamma_{A S}\left(1-\varepsilon_{S}\right) \sigma_{S} \beta_{S} b_{M} I_{M}^{* *} \mu_{H} S_{A}^{* *}+\gamma_{A S}\left(\beta_{S} b_{M} I_{M}^{* *} \mu_{H}+\pi_{j} \mu_{S}\right)\left[\left(1-\varepsilon_{S}\right) \sigma_{S} E_{A}^{* *}+I_{S A}^{* *} k_{11}\right]\right. \\
& +\gamma_{S S}\left(1-\varepsilon_{S}\right) \sigma_{S} \beta_{S} b_{M} I_{M}^{* *} \mu_{H} S_{A}^{* *}+\gamma_{S S}\left(\beta_{S} b_{M} I_{M}^{* *} \mu_{H}+\pi_{J} \mu_{S}\right)\left[\left(1-\varepsilon_{S}\right) \sigma_{S} E_{A}^{* * *}+I_{S A}^{* * *} k_{11}\right] \\
& \left.+R_{A}^{* *} k_{11} k_{13}\left(\beta_{S} b_{M} I_{M}^{* *} \mu_{H}+\pi_{J} \mu_{S}\right)\right\}\left[\mu_{S} k_{13} k_{11}\left(\beta_{S} b_{M} I_{M}^{* *} \mu_{H}+\pi_{J} \mu_{S}\right)\right]^{-1}, \\
& S_{M}^{* *}=\frac{\pi_{M}}{\left(\lambda_{M}^{* *}+\mu_{M}\right)}, \\
& E_{M}^{* *}=\frac{\lambda_{M}^{* *} \pi_{M}}{k_{14}\left(\lambda_{M}^{* *}+\mu_{M}\right)}, \\
& I_{M}^{* *}=\frac{\sigma_{M} \lambda_{M}^{* *} \pi_{M}}{k_{14} \mu_{M}\left(\lambda_{M}^{* *}+\mu_{M}\right)} .
\end{aligned}
$$

\section{G. Proof of Theorem 7}

Proof. The proof is based on using the centre manifold theory [51], as described in [58]. It is convenient to make the following simplification and change of variables.

Let $S_{J}=x_{1}, E_{J}=x_{2}, I_{A J}=x_{3}, I_{S J}=x_{4}, R_{J}=x_{5}, S_{A}=x_{6}$, $E_{A}=x_{7}, I_{A A}=x_{8}, I_{S A}=x_{9}, R_{A}=x_{10}, S_{S}=x_{11}, E_{S}=x_{12}$, $I_{A S}=x_{13}, I_{S S}=x_{14}, R_{S}=x_{15}, S_{M}=x_{16}, E_{M}=x_{17}$, and $I_{M}=x_{18}$ so that $N_{X}=x_{1}+x_{2}+x_{3}+x_{4}+x_{5}+x_{6}+x_{7}+x_{8}+x_{9}+$ $x_{10}+x_{11}+x_{12}+x_{13}+x_{14}+x_{15}$. Using the vector notation $\mathbf{x}=$ $\left(x_{1}, x_{2}, x_{3}, x_{4}, x_{5}, x_{6}, x_{7}, x_{8}, x_{9}, x_{10}, x_{11}, x_{12}, x_{13}, x_{14}, x_{15}, x_{16}\right.$, $\left.x_{17}, x_{18}\right)^{T}$, model (29) can be written in the form $d \mathbf{x} / d t=$ $\mathbf{m}(\mathbf{x})$, where $\mathbf{m}=\left(m_{1}, m_{2}, m_{3}, m_{4}, m_{5}, m_{6}, m_{7}, m_{8}, m_{9}, m_{10}\right.$, $\left.m_{11}, m_{12}, m_{13}, m_{14}, m_{15}, m_{16}, m_{17}, m_{18}\right)^{T}$, as follows:

$$
\begin{aligned}
& \frac{d x_{1}}{d t}=m_{1}=\pi_{J}-\frac{\beta_{J} b_{M} x_{18} x_{1}}{N_{X}}-k_{1} x_{1}, \\
& \frac{d x_{2}}{d t}=m_{2}=\frac{\beta_{J} b_{M} x_{18} x_{1}}{N_{X}}-k_{2} x_{2}, \\
& \frac{d x_{3}}{d t}=m_{3}=\varepsilon_{J} \sigma_{J} x_{2}-k_{3} x_{4},
\end{aligned}
$$




$$
\begin{aligned}
& \frac{d x_{4}}{d t}=m_{4}=\left(1-\varepsilon_{J}\right) \sigma_{J} x_{2}-k_{4} x_{3}, \\
& \frac{d x_{5}}{d t}=m_{5}=\gamma_{A J} x_{4}+\gamma_{S J} x_{3}-k_{5} x_{5}, \\
& \frac{d x_{6}}{d t}=m_{6}=\frac{\beta_{A} b_{M} x_{18} x_{6}}{N_{X}}-k_{6} x_{6}, \\
& \frac{d x_{7}}{d t}=m_{7}=\frac{\beta_{A} b_{M} x_{18} x_{6}}{N_{X}}-k_{7} x_{7}, \\
& \frac{d x_{8}}{d t}=m_{8}=\alpha x_{3}+\varepsilon_{A} \sigma_{A} x_{7}-k_{8} x_{8}, \\
& \frac{d x_{9}}{d t}=m_{9}=\alpha x_{4}+\left(1-\varepsilon_{A}\right) \sigma_{A} x_{7}-k_{9} x_{9}, \\
& \frac{d x_{10}}{d t}=m_{10}=\alpha x_{5}+\gamma_{A A} x_{8}+\gamma_{S A} x_{9}-k_{10} x_{10}, \\
& \frac{d x_{11}}{d t}=m_{11}=\xi x_{6}-\frac{\beta_{S} b_{M} x_{18} x_{11}}{N_{X}}-\mu_{H} x_{11}, \\
& \frac{d x_{12}}{d t}=m_{12}=\xi x_{7}+\frac{\beta_{S} b_{M} x_{18} x_{11}}{N_{X}}-k_{11} x_{12}, \\
& \frac{d x_{13}}{d t}=m_{13}=\xi x_{8}+\varepsilon_{S} \sigma_{S} x_{12}-k_{12} x_{13}, \\
& \frac{d t}{d t}=\xi x_{9}+\left(1-\varepsilon_{S}\right) \sigma_{S} x_{12}-k_{13} x_{14},
\end{aligned}
$$$$
\frac{d x_{15}}{d t}=m_{15}=\xi x_{10}+\gamma_{A S} x_{14}+\gamma_{S S} x_{14}-\mu_{S} x_{15},
$$

$$
\begin{aligned}
\frac{d x_{16}}{d t} & =m_{16} \\
= & \pi_{M} \\
& -\frac{\beta_{M} b_{M}\left(x_{3}+x_{4}+x_{8}+x_{9}+x_{13}+x_{14}\right) x_{16}}{N_{X}} \\
& -\mu_{M} x_{16},
\end{aligned}
$$$$
\frac{d x_{17}}{d t}=m_{17}
$$$$
=\frac{\beta_{M} b_{M}\left(x_{3}+x_{4}+x_{8}+x_{9}+x_{13}+x_{14}\right) x_{16}}{N_{X}}
$$$$
-k_{14} x_{17}
$$$$
\frac{d x_{18}}{d t}=m_{18}=\sigma_{M} x_{17}-\mu_{M} x_{18}
$$

The Jacobian of the transformed system (G.1) at the diseasefree equilibrium $\mathscr{E}_{1}$, is given by

$$
J\left(\mathscr{E}_{1}\right)=\left(J_{1} \mid J_{2}\right)
$$

where

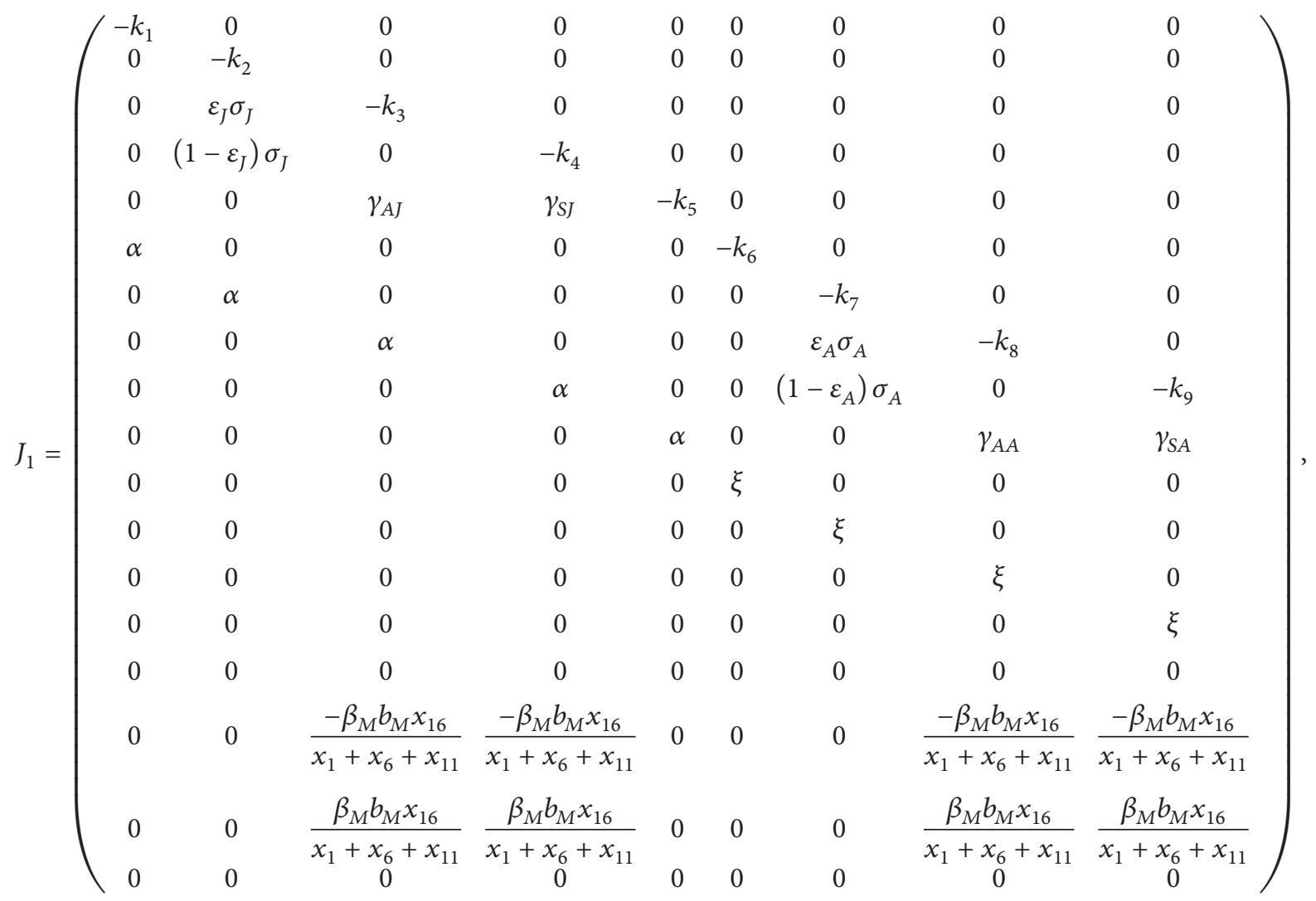




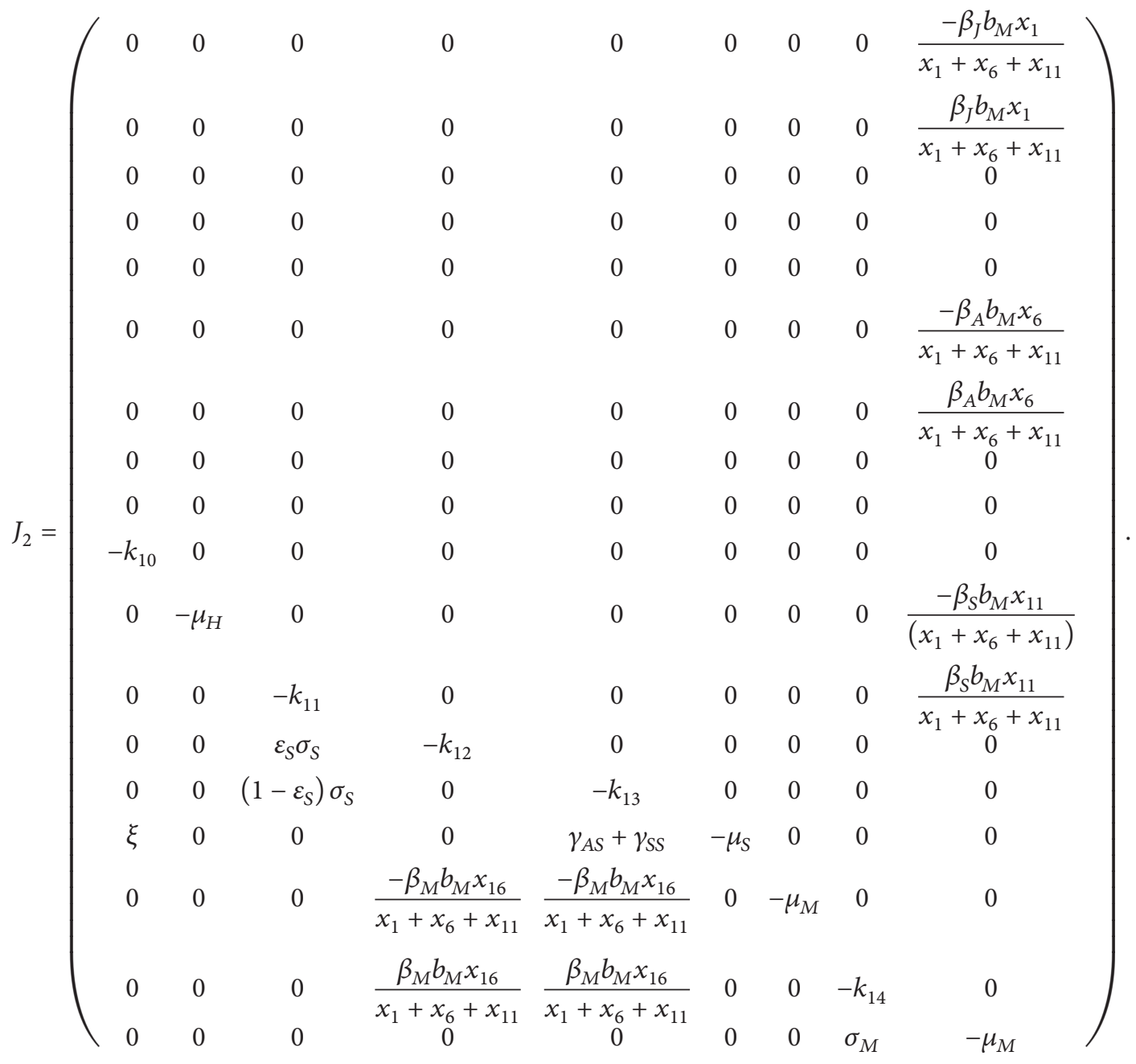

Consider the case when $\mathscr{R}_{0}=1$. Suppose, further, that $\beta_{M}$ is chosen as a bifurcation parameter. Solving (29) for $\beta_{M}$ from $\mathscr{R}_{0}=1$ gives $\beta_{M}=\beta_{M}^{*}$. The transformed system (G.1) at the DFE evaluated at $\beta_{M}=\beta_{M}^{*}$ has a simple zero eigenvalue (and all other eigenvalues having negative real parts). Hence, the centre manifold theory [51] can be used to analyze the dynamics of (G.1) near $\beta_{M}=\beta_{M}^{*}$. In particular, the theorem in [58] (see also $[42,51,52]$ ) is used. To apply the theorem, the following computations are necessary (it should be noted that we are using $\beta_{M}$ instead of $\phi$ for the bifurcation parameter).

Eigenvectors of $\left.J\left(\mathscr{E}_{1}\right)\right|_{\beta_{M}=\beta_{M}^{*}}$. The Jacobian of (G.1) at $\beta_{M}=$ $\beta_{M}^{*}$, denoted by $\left.J\left(\mathscr{E}_{1}\right)\right|_{\beta_{M}=\beta_{M}^{*}}$, has a right eigenvector (associated with the zero eigenvalue) given by

$$
\begin{gathered}
\mathbf{w}=\left(w_{1}, w_{2}, w_{3}, w_{4}, w_{5}, w_{6}, w_{7}, w_{8}, w_{9}, w_{10}, w_{11}, w_{12},\right. \\
\left.w_{13}, w_{14}, w_{15}, w_{16}, w_{17}, w_{18}\right)^{T},
\end{gathered}
$$

where

$$
w_{1}=\frac{-\beta_{J} b_{M} w_{18} x_{1}}{k_{1}\left(x_{1}+x_{6}+x_{11}\right)},
$$

$$
\begin{aligned}
& w_{2}=\frac{\beta_{J} b_{M} w_{18} x_{1}}{k_{2}\left(x_{1}+x_{6}+x_{11}\right)}, \\
& w_{3}=\frac{\varepsilon_{J} \sigma_{J} w_{2}}{k_{3}}, \\
& w_{4}=\frac{\left(1-\varepsilon_{J}\right) \sigma_{J} w_{2}}{k_{4}}, \\
& w_{5}=\frac{\left(\gamma_{A J} w_{3}+\gamma_{S J} w_{4}\right)}{k_{5}}, \\
& w_{6}=\frac{1}{k_{6}}\left(\alpha w_{1}-\frac{b_{M} \beta_{A} w_{18} x_{6}}{x_{1}+x_{6}+x_{11}}\right), \\
& w_{7}=\frac{1}{k_{7}}\left(\alpha w_{2}+\frac{b_{M} \beta_{A} w_{18} x_{6}}{x_{1}+x_{6}+x_{11}}\right), \\
& w_{8}=\frac{\alpha w_{3}+\varepsilon_{A} \sigma_{A} w_{7}}{k_{8}}, \\
& w_{9}=\frac{\alpha w_{4}+\left(1-\varepsilon_{A}\right) \sigma_{A} w_{7}}{k_{9}},
\end{aligned}
$$




$$
\begin{aligned}
& w_{10}=\frac{\alpha w_{5}+\gamma_{A A} w_{8}+\gamma_{S A} w_{9}}{k_{10}}, \\
& w_{11}=\frac{1}{\mu_{S}}\left(\xi w_{6}-\frac{b_{M} \beta_{S} w_{18} x_{11}}{x_{1}+x_{6}+x_{11}}\right), \\
& w_{12}=\frac{1}{k_{11}}\left(\xi w_{7}+\frac{b_{M} \beta_{S} w_{18} x_{11}}{x_{1}+x_{6}+x_{11}}\right) \text {, } \\
& w_{13}=\frac{\xi w_{8}+\varepsilon_{S} \sigma_{S} w_{12}}{k_{12}} \text {, } \\
& w_{14}=\frac{\xi w_{9}+\left(1-\varepsilon_{S}\right) \sigma_{S} w_{12}}{k_{13}}, \\
& w_{15}=\frac{\xi w_{10}+\left(\gamma_{A A}+\gamma_{S A}\right) w_{14}}{\mu_{S}}, \\
& w_{16} \\
& =\frac{-b_{M} \beta_{M} x_{16}}{\mu_{M}}\left(\frac{w_{3}+w_{4}+w_{8}+w_{9}+w_{13}+w_{14}}{x_{1}+x_{6}+x_{11}}\right), \\
& w_{17} \\
& =\frac{x_{11} b_{M} \beta_{M}}{k_{9}}\left(\frac{w_{3}+w_{4}+w_{8}+w_{9}+w_{13}+w_{14}}{x_{1}+x_{6}+x_{11}}\right), \\
& w_{18}=\frac{\sigma_{M}}{\mu_{M}} \text {. }
\end{aligned}
$$

Also, $\left.J\left(\mathscr{E}_{1}\right)\right|_{\beta_{M}=\beta_{M}^{*}}$ has a left eigenvector $\mathbf{v}=\left(v_{1}, v_{2}, v_{3}, v_{4}\right.$, $v_{5}, v_{6}, v_{7}, v_{8}, v_{9}, v_{10}, v_{11}, v_{12}, v_{13}, v_{14}, v_{15}, v_{16}, v_{17}, v_{18}$ ) (associated with the zero eigenvalue), where

$$
\begin{aligned}
& v_{1}=0 \\
& v_{2}=\frac{\varepsilon_{J} \sigma_{J} v_{3}+\left(1-\varepsilon_{J}\right) \sigma_{J} v_{4}+\alpha v_{7}}{k_{2}}, \\
& v_{3}=\frac{1}{k_{3}}\left(\alpha v_{8}+\frac{x_{11} b_{M} \beta_{M} v_{17}}{x_{1}+x_{6}+x_{11}}\right), \\
& v_{4}=\frac{1}{k_{4}}\left(\alpha v_{9}+\frac{x_{11} b_{M} \beta_{M} v_{17}}{x_{1}+x_{6}+x_{11}}\right), \\
& v_{5}=0 \\
& v_{6}=0 \\
& v_{7}=\frac{\varepsilon_{A} \sigma_{A} v_{8}+\left(1-\varepsilon_{A}\right) \sigma_{A} v_{9}+\xi v_{12}}{k_{7}}, \\
& v_{8}=\frac{1}{k_{8}}\left(\xi v_{13}+\frac{x_{11} b_{M} \beta_{M} v_{17}}{x_{1}+x_{6}+x_{11}}\right), \\
& v_{9}=\frac{1}{k_{9}}\left(\xi v_{14}+\frac{x_{11} b_{M} \beta_{M} v_{17}}{x_{1}+x_{6}+x_{11}}\right), \\
& =0
\end{aligned}
$$

$$
\begin{aligned}
& v_{11}=0, \\
& v_{15}=0, \\
& v_{16}=0, \\
& v_{12}=\frac{\varepsilon_{S} \sigma_{S} v_{13}+\left(1-\varepsilon_{S}\right) \sigma_{S} v_{14}}{k_{11}}, \\
& v_{13}=\frac{x_{11} b_{M} \beta_{M} v_{17}}{k_{11}\left(x_{1}+x_{6}+x_{11}\right)}, \\
& v_{14}=\frac{x_{11} b_{M} \beta_{M} v_{17}}{k_{13}\left(x_{1}+x_{6}+x_{11}\right)}, \\
& v_{17}=\frac{\sigma_{M} v_{18}}{k_{14}}, \\
& v_{18}>0 .
\end{aligned}
$$

Computations of Bifurcation Coefficients $a$ and $b$. The application of the theorem in [58] entails the computation of two bifurcation coefficients $a$ and $b$. It can be shown, after some algebraic manipulations, that

$$
a=v_{2} \sum_{i, j=1}^{18} w_{i} w_{j} \frac{\partial^{2} f_{2}}{\partial x_{i} \partial x_{j}}+v_{7} \sum_{i, j=1}^{18} w_{i} w_{j} \frac{\partial^{2} f_{7}}{\partial x_{i} \partial x_{j}}
$$

$$
\begin{aligned}
& +v_{12} \sum_{i, j=1}^{18} w_{i} w_{j} \frac{\partial^{2} f_{12}}{\partial x_{i} \partial x_{j}}+v_{17} \sum_{i, j=1}^{18} w_{i} w_{j} \frac{\partial^{2} f_{17}}{\partial x_{i} \partial x_{j}} \\
& =\frac{2 b_{M}}{\left(x_{1}+x_{6}+x_{11}\right)^{2}}\left[w _ { 1 8 } \left(\left(x_{6}+x_{11}\right) v_{2} w_{1} \beta_{J}\right.\right. \\
& \left.+\left(x_{1}+x_{11}\right) v_{7} w_{6} \beta_{A}+\left(x_{1}+x_{6}\right) v_{12} w_{11} \beta_{S}\right) \\
& +v_{17} w_{16} \beta_{M}\left(w_{3}+w_{8}+w_{9}+w_{13}+w_{14}\right)\left(x_{1}+x_{6}\right. \\
& \left.+x_{11}\right)-w_{18}\left[\left(w_{6}+w_{11}\right) v_{2} x_{1} \beta_{J}\right. \\
& \left.+\left(w_{1}+w_{11}\right) v_{7} x_{6} \beta_{A}+\left(w_{1}+w_{6}\right) v_{12} x_{11} \beta_{S}\right] \\
& -w_{18}\left(w_{2}+w_{3}+w_{5}+w_{7}+w_{8}+w_{9}+w_{10}\right. \\
& \left.+w_{12}+w_{13}+w_{14}+w_{15}\right)\left(v_{2} x_{1} \beta_{J}+v_{7} x_{6} \beta_{A}\right. \\
& \left.+v_{12} x_{11} \beta_{S}\right)-v_{17} x_{16} \beta_{M}\left(w_{3}+w_{8}+w_{9}+w_{13}\right. \\
& \left.+w_{14}\right)\left(w_{1}+w_{2}+w_{3}+w_{5}+w_{6}+w_{7}+w_{8}+w_{9}\right. \\
& \left.\left.+w_{10}+w_{11}+w_{12}+w_{13}+w_{14}+w_{15}\right)\right] .
\end{aligned}
$$

Furthermore,

$$
\begin{aligned}
b & =v_{17} \sum_{i=1}^{18} w_{i} \frac{\partial^{2} f_{17}}{\partial x_{i} \partial \beta_{p}^{*}} \\
& =\frac{v_{17} x_{16} b_{M}\left(w_{3}+w_{8}+w_{9}+w_{13}+w_{14}\right)}{\left(x_{1}+x_{6}+x_{11}\right)}>0 .
\end{aligned}
$$


Hence, it follows from Theorem 4.1 of [58] that the transformed model (G.1) (or, equivalently, (10)) undergoes backward bifurcation at $\mathscr{R}_{0}=1$ whenever the following inequality holds:

$$
a>0 .
$$

\section{Conflict of Interests}

The authors declare that there is no conflict of interests regarding the publication of this paper.

\section{References}

[1] Centers for Disease Control and Prevention, Chikungunya Virus, Centers for Disease Control and Prevention, 2014, http:// www.cdc.gov/chikungunya/symptoms/index.html.

[2] World Health Organization, "Chikungunya," 2014, http://www .who.int/denguecontrol/arbo-viral/other_arboviral_chikungunya/en/.

[3] World Health Organization, “Chikungunya," 2014, http://www .who.int/mediacentre/factsheets/fs327/en/.

[4] M. Doucleff, "Trouble in paradise: chikungunya outbreak grows in Caribbean," Health News, 2013.

[5] G. Pialoux, B.-A. Gaüzère, S. Jauréguiberry, and M. Strobel, "Chikungunya, an epidemic arbovirosis," The Lancet Infectious Diseases, vol. 7, no. 5, pp. 319-327, 2007.

[6] M. Dubrulle, L. Mousson, S. Moutailier, M. Vazeille, and A.-B. Failloux, "Chikungunya virus and Aedes mosquitoes: saliva is infectious as soon as two days after oral infection," PLOS ONE, vol. 4, no. 6, Article ID e5895, 2009.

[7] I. Schuffenecker, I. Iteman, A. Michault et al., "Genome microevolution of chikungunya viruses causing the Indian Ocean outbreak," PLoS Medicine, vol. 3, no. 7, pp. 1058-1070, 2006.

[8] M. Vazeille, S. Moutailler, D. Coudrier et al., "Two Chikungunya isolates from the outbreak of La Reunion (Indian Ocean) exhibit different patterns of infection in the mosquito, Aedes albopictus," PLoS ONE, vol. 2, no. 11, Article ID el168, 2007.

[9] M. Enserink, "Epidemiology. Tropical disease follows mosquitoes to Europe," Science, vol. 317, no. 5844, p. 1485, 2007.

[10] R. K. Singh, S. Tiwari, V. K. Mishra, R. Tiwari, and T. N. Dhole, "Molecular epidemiology of Chikungunya virus: mutation in E1 gene region," Journal of Virological Methods, vol. 185, no. 2, pp. 213-220, 2012.

[11] K. A. Tsetsarkin, D. L. Vanlandingham, C. E. McGee, and S. Higgs, "A single mutation in Chikungunya virus affects vector specificity and epidemic potential," PLoS Pathogens, vol. 3, no. 12, article e201, 2007.

[12] L.-J. Chang, K. A. Dowd, F. H. Mendoza et al., "Safety and tolerability of chikungunya virus-like particle vaccine in healthy adults: a phase 1 dose-escalation trial," The Lancet, vol. 384, no. 9959, pp. 2046-2052, 2014.

[13] M. Kannan, R. Rajendran, I. P. Sunish et al., "A study on chikungunya outbreak during 2007 in Kerala, South India," Indian Journal of Medical Research, vol. 129, no. 3, pp. 311-315, 2009.

[14] A. Mohan, D. H. N. Kiran, I. Manohar, and D. P. Kumar, "Epidemiology, clinical manifestations, and diagnosis of chikungunya fever: lessons learned from the re-emerging epidemic," Indian Journal of Dermatology, vol. 55, no. 1, pp. 54-63, 2010.
[15] T. E. Morrison, "Reemergence of chikungunya virus," Journal of Virology, vol. 88, no. 20, pp. 11644-11647, 2014.

[16] N. Ritz, M. Hufnagel, and P. Grardin, "Chikungunya in children," ESPID Reports and Reviews Pediatric Infectious Disease Journal, vol. 34, no. 7, pp. 789-791, 2015.

[17] J. E. Staples, S. L. Hills, and A. M. Powers, Chikungunya, Centers for Disease Control and Prevention (CDC), 2014, http://wwwnc .cdc.gov/travel/yellowbook/2014/chapter-3-infectious-diseasesrelated-to-travel/chikungunya.

[18] W. Van Bortel, F. Dorleans, J. Rosine et al., "Chikungunya outbreak in the Caribbean region, December 2013 to March 2014, and the significance for Europe," Eurosurveillance, vol. 19, no. 13, 2014.

[19] K. P. Vijayakumar, T. S. Nair Anish, B. George, T. Lawrence, S. C. Muthukkutty, and R. Ramachandran, "Clinical profile of chikungunya patients during the epidemic of 2007 in Kerala, India," Journal of Global Infectious Diseases, vol. 3, no. 3, pp. 221226, 2011.

[20] V. Lakshmi, M. Neeraja, M. V. S. Subbalaxmi et al., "Clinical features and molecular diagnosis of Chikungunya fever from South India," Clinical Infectious Diseases, vol. 46, no. 9, pp. 14361442, 2008.

[21] P. Pongsumpun and S. Sangsawang, "Local stability analysis for age structural model of chikungunya disease," Journal of Basic and Applied Scientific Research, vol. 3, no. 3, pp. 302-312, 2013.

[22] G. Pialoux, B.-A. Gaüzère, and M. Strobel, "Chikungunya virus infection: review through an epidemic," Medecine et Maladies Infectieuses, vol. 36, no. 5, pp. 253-263, 2006.

[23] European Centre for Disease Prevention and Control (ECDC), Annual Epidemiological Report 2014-Emerging and VectorBorne Diseases, European Centre for Disease Prevention and Control (ECDC), 2014, http://ecdc.europa.eu/en/publications/ Publications/emerging-vector-borne-diseases_annual-epidemiological-report-2014.pdf.

[24] D. Ruiz-Moreno, I. S. Vargas, K. E. Olson, and L. C. Harrington, "Modeling dynamic introduction of Chikungunya virus in the United States," PLoS Neglected Tropical Diseases, vol. 6, no. 11, Article ID e1918, 2012.

[25] Y. Dumont, F. Chiroleu, and C. Domerg, "On a temporal model for the Chikungunya disease: modeling, theory and numerics," Mathematical Biosciences, vol. 213, no. 1, pp. 80-91, 2008.

[26] C. A. Manore, K. S. Hickmann, S. Xu, H. J. Wearing, and J. M. Hyman, "Comparing dengue and chikungunya emergence and endemic transmission in A. aegypti and A. albopictus," Journal of Theoretical Biology, vol. 356, no. 7, pp. 174-191, 2014.

[27] O. P. Misra and D. K. Mishra, "Simultaneous effects of control measures on the transmission dynamics of Chikungunya disease," Journal Applied Mathematics, vol. 2, no. 4, pp. 124-130, 2012.

[28] D. Moulay, M. A. Aziz-Alaoui, and M. Cadivel, "The chikungunya disease: modeling, vector and transmission global dynamics," Mathematical Biosciences, vol. 229, no. 1, pp. 50-63, 2011.

[29] P. Poletti, G. Messeri, M. Ajelli, R. Vallorani, C. Rizzo, and S. Merler, "Transmission potential of Chikungunya virus and control measures: the case of Italy," PLoS ONE, vol. 6, no. 5, Article ID e18860, 2011.

[30] L. Yakob and A. C. A. Clements, "A mathematical model of chikungunya dynamics and control: the major epidemic on Réunion Island," PLoS ONE, vol. 8, no. 3, Article ID e57448, 2013. 
[31] N. Chitnis, J. M. Hyman, and J. M. Cushing, "Determining important parameters in the spread of malaria through the sensitivity analysis of a mathematical model," Bulletin of Mathematical Biology, vol. 70, no. 5, pp. 1272-1296, 2008.

[32] Y. Dumont and F. Chiroleu, "Vector control for the Chikungunya disease," Mathematical Biosciences and Engineering, vol. 7, no. 2, pp. 313-345, 2010.

[33] M. J. Turell, J. R. Beaman, and R. F. Tammariello, "Susceptibility of selected strains of Aedes aegypti and Aedes albopictus (Diptera: Culicidae) to chikungunya virus," Journal of Medical Entomology, vol. 29, no. 1, pp. 49-53, 1992.

[34] H. Delatte, G. Gimonneau, A. Triboire, and D. Fontenille, "Influence of temperature on immature development, survival, longevity, fecundity, and gonotrophic cycles of aedes albopictus, vector of chikungunya and dengue in the Indian Ocean," Journal of Medical Entomology, vol. 46, no. 1, pp. 33-41, 2009.

[35] C. Lahariya and S. K. Pradhan, "Emergence of chikungunya virus in Indian subcontinent after 32 years: a review," Journal of Vector Borne Diseases, vol. 43, no. 4, pp. 151-160, 2006.

[36] M. R. Sebastian, R. Lodha, and S. K. Kabra, "Chikungunya infection in children," Indian Journal of Pediatrics, vol. 76, no. 2, pp. 185-189, 2009.

[37] O. Schwartz and M. L. Albert, "Biology and pathogenesis of chikungunya virus," Nature Reviews Microbiology, vol. 8, no. 7, pp. 491-500, 2010.

[38] K. S. Costanzo, K. Mormann, and S. A. Juliano, "Asymmetrical competition and patterns of abundance of Aedes albopictus and Culex pipiens (Diptera: Culicidae)," Journal of Medical Entomology, vol. 42, no. 4, pp. 559-570, 2005.

[39] H. Nur Aida, A. Abu Hassan, A. T. Nurita, M. R. Che Salmah, and B. Norasmah, "Population analysis of aedes albopictus (Skuse) (Diptera: Culicidae) under uncontrolled laboratory conditions," Tropical Biomedicine, vol. 25, no. 2, pp. 117-125, 2008.

[40] E. Massad, S. Ma, M. N. Burattini, Y. Tun, F. A. B. Coutinho, and L. W. Ang, "The risk of chikungunya fever in a dengue-endemic area," Journal of Travel Medicine, vol. 15, no. 3, pp. 147-155, 2008.

[41] K. Pesko, C. J. Westbrook, C. N. Mores, L. P. Lounibos, and M. H. Reiskind, "Effects of infectious virus dose and bloodmeal delivery method on susceptibility of Aedes aegypti and Aedes albopictus to chikungunya virus," Journal of Medical Entomology, vol. 46, no. 2, pp. 395-399, 2009.

[42] P. van den Driessche and J. Watmough, "Reproduction numbers and sub threshold endemic equilibria for compartmental models of disease transmission," Mathematical Biosciences, vol. 180, pp. 29-48, 2002.

[43] R. M. Anderson and R. May, Infectious Diseases of Humans, Oxford University Press, New York, NY, USA, 1991.

[44] O. Diekmann, J. A. P. Heesterbeek, and J. A. P. Metz, "On the definition and the computation of the basic reproduction ratio $R_{0}$ in models for infectious diseases in heterogeneous populations," Journal of Mathematical Biology, vol. 28, no. 4, pp. 365382, 1990.

[45] H. W. Hethcote, "The mathematics of infectious diseases," SIAM Review, vol. 42, no. 4, pp. 599-653, 2000.

[46] P. Gérardin, V. Guernier, J. Perrau et al., "Estimating Chikungunya prevalence in La Réunion Island outbreak by serosurveys: two methods for two critical times of the epidemic," $B M C$ Infectious Diseases, vol. 8, article 99, 2008.
[47] P. Renault, J.-L. Solet, D. Sissoko et al., "A major epidemic of chikungunya virus infection on Réunion Island, France, 20052006," The American Journal of Tropical Medicine and Hygiene, vol. 77, no. 4, pp. 727-731, 2007.

[48] F. B. Agusto and A. B. Gumel, "Qualitative dynamics of lowlyand highly-pathogenic avian influenza strains," Mathematical Biosciences, vol. 243, no. 2, pp. 147-162, 2013.

[49] F. B. Agusto, S. Lenhart, A. B. Gumel, and A. Odoi, "Mathematical analysis of a model for the transmission dynamics of bovine tuberculosis," Mathematical Methods in the Applied Sciences, vol. 34, no. 15, pp. 1873-1887, 2011.

[50] F. Brauer, "Backward bifurcations in simple vaccination models," Journal of Mathematical Analysis and Applications, vol. 298, no. 2, pp. 418-431, 2004.

[51] J. Carr, Applications of Centre Manifold Theory, Springer, New York, NY, USA, 1981.

[52] J. Dushoff, W. Huang, and C. Castillo-Chavez, "Backwards bifurcations and catastrophe in simple models of fatal diseases," Journal of Mathematical Biology, vol. 36, no. 3, pp. 227-248, 1998.

[53] E. H. Elbasha and A. B. Gumel, "Theoretical assessment of public health impact of imperfect prophylactic HIV-1 vaccines with therapeutic benefits," Bulletin of Mathematical Biology, vol. 68, no. 3, pp. 577-614, 2006.

[54] S. M. Garba and A. B. Gumel, "Effect of cross-immunity on the transmission dynamics of two strains of dengue," International Journal of Computer Mathematics, vol. 87, no. 10, pp. 2361-2384, 2010.

[55] S. M. Garba, A. B. Gumel, and M. R. Abu Bakar, "Backward bifurcations in dengue transmission dynamics," Mathematical Biosciences, vol. 215, no. 1, pp. 11-25, 2008.

[56] O. Sharomi, C. N. Podder, A. B. Gumel, E. H. Elbasha, and J. Watmough, "Role of incidence function in vaccine-induced backward bifurcation in some HIV models," Mathematical Biosciences, vol. 210, no. 2, pp. 436-463, 2007.

[57] O. Sharomi, C. N. Podder, A. B. Gumel, and B. Song, "Mathematical analysis of the transmission dynamics of HIV/TB coinfection in the presence of treatment," Mathematical Biosciences and Engineering, vol. 5, no. 1, pp. 145-174, 2008.

[58] C. Castillo-Chavez and B. Song, "Dynamical models of tuberculosis and their applications," Mathematical Biosciences and Engineering, vol. 1, no. 2, pp. 361-404, 2004.

[59] F. Forouzannia and A. B. Gumel, "Mathematical analysis of an age-structured model for malaria transmission dynamics," Mathematical Biosciences, vol. 247, pp. 80-94, 2014.

[60] S. M. Blower and H. Dowlatabadi, "Sensitivity and uncertainty analysis of complex models of disease transmission: an HIV model, as an example," International Statistical Review, vol. 62, no. 2, pp. 229-243, 1994.

[61] S. Marino, I. B. Hogue, C. J. Ray, and D. E. Kirschner, "A methodology for performing global uncertainty and sensitivity analysis in systems biology," Journal of Theoretical Biology, vol. 254, no. 1, pp. 178-196, 2008.

[62] R. G. McLeod, J. F. Brewster, A. B. Gumel, and D. A. Slonowsky, "Sensitivity and uncertainty analyses for a SARS model with time-varying inputs and outputs," Mathematical Biosciences and Engineering, vol. 3, no. 3, pp. 527-544, 2006.

[63] Wikipedia, "Demographics of India," 2015, https://en.wikipedia .org/wiki/Demographics_of_India.

[64] F. B. Agusto, "Optimal isolation control strategies and costeffectiveness analysis of a two-strain avian influenza model," BioSystems, vol. 113, no. 3, pp. 155-164, 2013. 
[65] C. Bowman, A. B. Gumel, P. van den Driessche, J. Wu, and H. Zhu, "A mathematical model for assessing control strategies against West Nile virus," Bulletin of Mathematical Biology, vol. 67, no. 5, pp. 1107-1133, 2005.

[66] A. M. Niger and A. B. Gumel, "Mathematical analysis of the role of repeated exposure on malaria transmission dynamics," Differential Equations and Dynamical Systems, vol. 16, no. 3, pp. 251-287, 2008.

[67] V. Lakshmikantham, S. Leela, and A. A. Martynyuk, Stability Analysis of Nonlinear Systems, Marcel Dekker, New York, NY, USA, 1989.

[68] F. B. Agusto, S. Y. Del Valle, K. W. Blayneh et al., "The impact of bed-net use on malaria prevalance," Journal of Theoretical Biology, vol. 320, pp. 58-65, 2013.

[69] C. Castillo-Chavez, S. Blower, P. van den Driessche, D. Kirschner, and A.-A. Yakubu, Mathematical Approaches for Emerging and Reemerging Infectious Diseases, Springer, New York, NY, USA, 2002. 


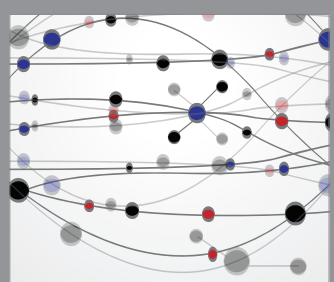

The Scientific World Journal
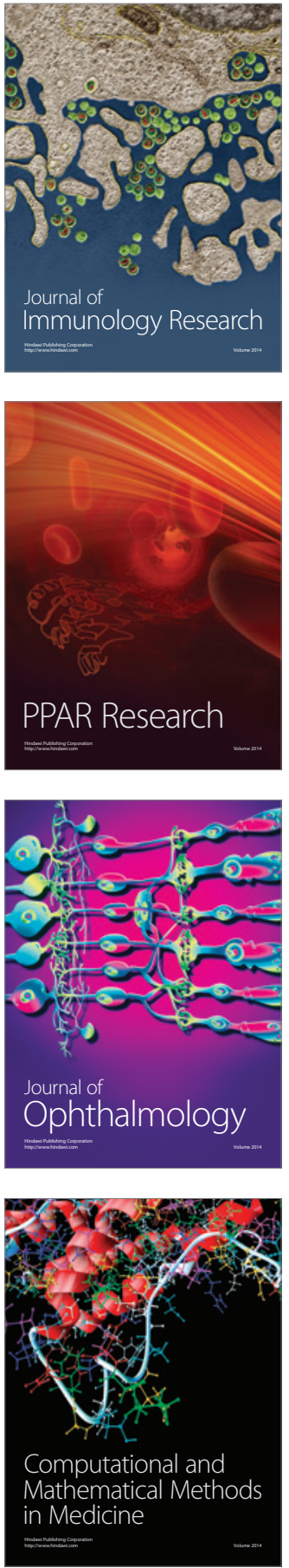

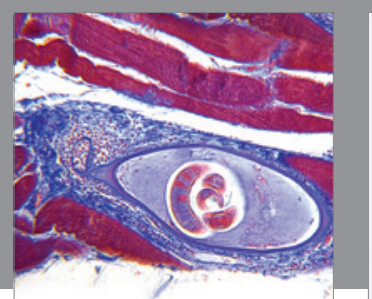

Gastroenterology Research and Practice

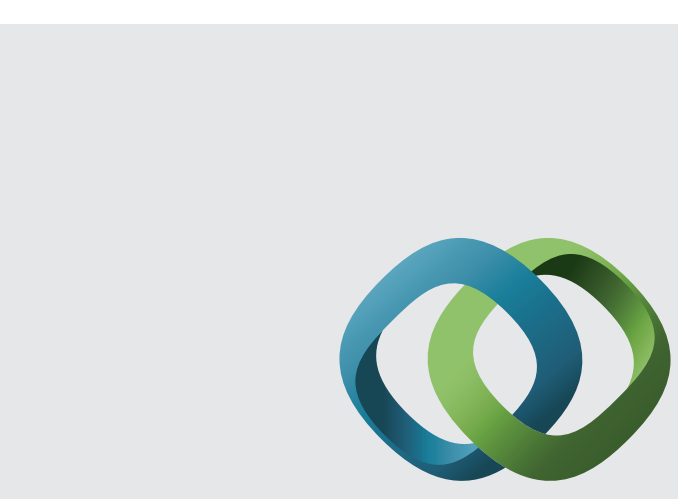

\section{Hindawi}

Submit your manuscripts at

http://www.hindawi.com
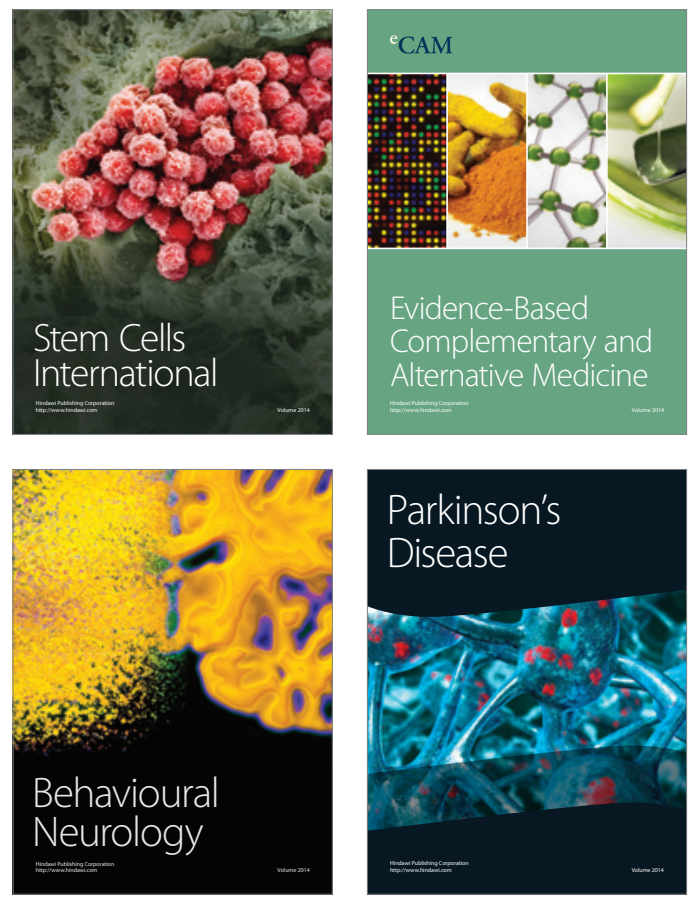
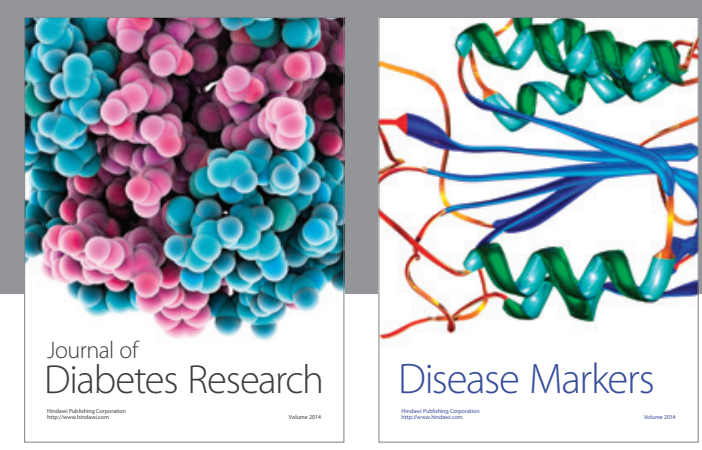

Disease Markers
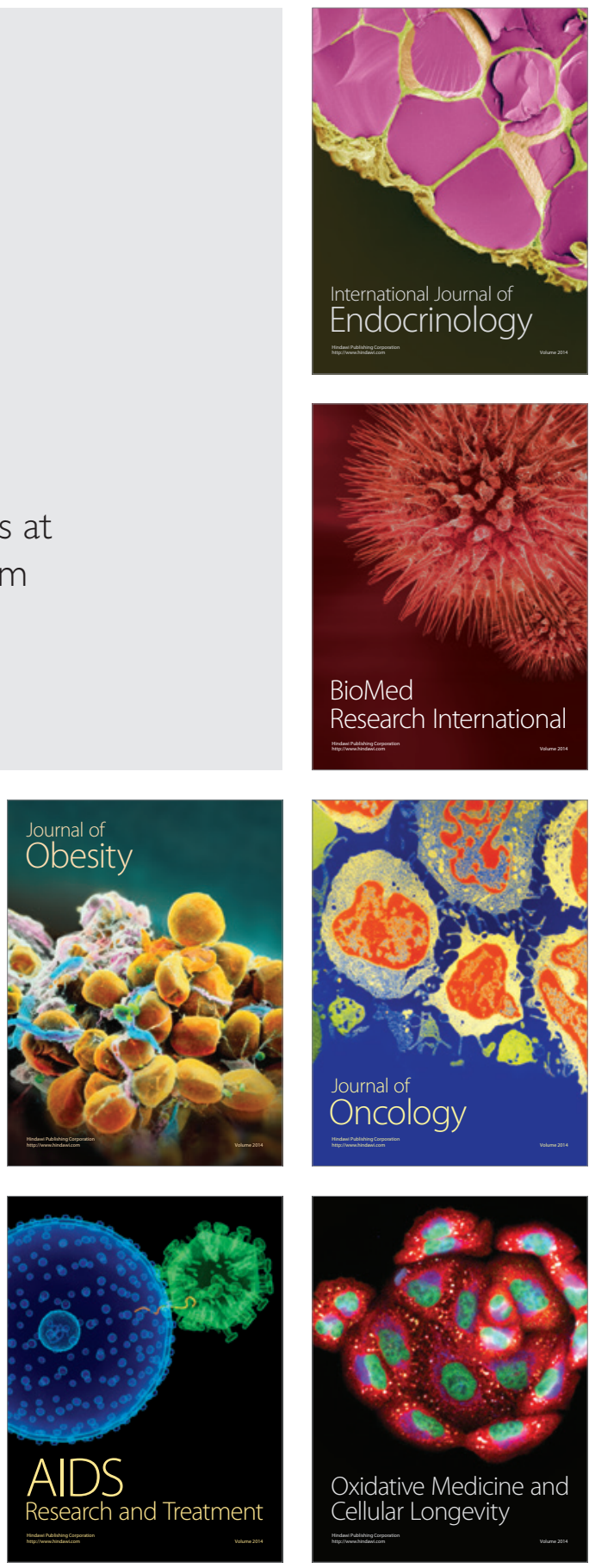